\title{
The HSPG Syndecan is a core organizer of cholinergic synapses in C. elegans
}

Xin ZHOU a, c, Camille VACHONa, c, Mélissa CIZERONa, Océane ROMATIFa, Hannes E. BÜLOW ${ }^{b}$, Maëlle JOSPIN ${ }^{a}$, Jean-Louis BESSEREAUa, d

(a) Univ Lyon, Université Claude Bernard Lyon 1, CNRS UMR 5310, INSERM U 1217, Institut NeuroMyoGène, 69008 Lyon, France.

(b) Department of Genetics \& Dominick P. Purpura Department of Neuroscience, Albert Einstein College of Medicine, Bronx, NY 10461, United States.

(c) Co-first authors

(d) Corresponding author

Corresponding author Jean-Louis BESSEREAU

Institut NeuroMyoGene

Laboratory of Genetics and Neurobiology of $C$. elegans

Univ Lyon, Université Claude Bernard Lyon 1, CNRS UMR 5310, INSERM U 1217,

Faculté de Médecine et de Pharmacie, 3ème étage-Aile $D$

8, Avenue Rockefeller

69008 Lyon - France.

tel. $+33 \quad 426688297$

email: jean-louis.bessereau@univ-lyon1.fr 


\section{SUMMARY}

The extracellular matrix has emerged as an active component of chemical synapses regulating synaptic formation, maintenance and homeostasis. The heparan sulfate proteoglycan syndecans are known to regulate cellular and axonal migration in the brain. They are also enriched at synapses, but their synaptic functions remain more elusive. Here we show that SDN-1, the sole ortholog of syndecan in C. elegans, is absolutely required for the synaptic clustering of homomeric $\alpha 7$-like $\mathrm{N}$-acetylcholine receptors (AChR) and regulates the synaptic content of heteromeric L-AChRs. SDN-1 is concentrated at neuromuscular junctions (NMJs) by the neurally-secreted synaptic organizer Ce-Punctin/MADD-4, which also activates the transmembrane netrin receptor DCC. Those cooperatively recruit the FARP and CASK orthologues that localize N-AChRs at cholinergic NMJs through physical interactions. Therefore, SDN-1 stands at the core of the cholinergic synapse organization by bridging the extracellular synaptic determinants to the intracellular synaptic scaffold that controls the postsynaptic receptor content.

\section{KEYWORDS}

Heparan Sulfate Proteoglycan; syndecan; synapse; ionotropic acetylcholine receptor; C. elegans; Punctin; DCC; postsynaptic scaffold; synaptomatrix; Autism Spectrum Disorder 


\section{INTRODUCTION}

Chemical synapses are specialized cellular junctions that support directional transfer of information between excitable cells. However, glial cells, by engaging intimate contacts with neuronal components at synaptic sites, were identified as important synaptic players, leading to the notion of the 'tripartite synapse' (Araque et al., 1999; Halassa et al., 2007). In addition, the extracellular matrix (ECM) that fills the synaptic cleft and the perisynaptic extracellular space emerged as an active element that orchestrates synaptogenesis, synaptic maintenance and synaptic function, thus leading to the concept of the 'tetrapartite synapse' (Dityatev and Rusakov, 2011; Dityatev et al., 2006; Ferrer-Ferrer and Dityatev, 2018). The synaptic ECM, also referred to as 'synaptomatrix' (Dani and Broadie, 2012; Heikkinen et al., 2014), forms a dense and composite environment. It is made of secreted glycoproteins and proteoglycans intermingled with the extracellular regions of pre- and postsynaptic membrane proteins and outer-leaflet glycolipids. Signaling between pre- and postsynaptic partners necessarily occurs through this specific environment, which consequently impacts all aspects of synaptic biology (Heikkinen et al., 2014; Kurshan et al., 2014).

Within the synaptomatrix, some glycoproteins such as heparan sulfate proteoglycans (HSPG) fulfill the role of bona fide synaptic organizers (Condomitti and de Wit, 2018a; Yuzaki, 2018). HSPGs are either secreted or membrane-associated core proteins bearing covalently-linked chains of long disaccharide repeats (Sarrazin et al., 2011). Enzymatic modifications of the glycan chains, including epimerization, deacetylations and sulfation at various positions, creates highly-negatively charged chains with distinct structural motifs (Lindahl and Li, 2009). HSPGs bind multiple partners and can promote or regulate synaptogenesis (Song and Kim 2013). The founding member of this diverse group is agrin, a large HSPG secreted by motoneurons at the vertebrate neuromuscular junctions (NMJs), which is absolutely required for NMJ differentiation and postsynaptic clustering of acetylcholine receptors (AChRs) (Burden et al., 2018; Li et al., 2018; Swenarchuk, 2019). Since then, an increasing number of HSPGs have been demonstrated to function as synaptic regulators (reviewed in Condomitti and de Wit, 2018). For example, in the hippocampus, the GPI-anchored glypican GPC4 binds the postsynaptic adhesion molecule LRRTM4 (de Wit et al., 2013) or the orphan receptor GPR158 (Condomitti et al., 2018) and promotes presynaptic differentiation through LAR-family receptor protein tyrosine phosphatases (RPTP). At other synapses, GPC4 is secreted by astrocytes and binds presynaptic RPTP $\delta$ and RPTP $\sigma$ to trigger the release of neuronal pentraxin 1, which in turn increases AMPAR content (Allen et al., 2012; Farhy-Tselnicker et al., 2017).

Syndecans are a class of transmembrane HSPGs also shown to play roles at synapses(SaiedSantiago and Bülow, 2018). In Drosophila, syndecan localizes to glutamatergic NMJs. It competes with the glypican Dallylike for binding to the LAR-family receptor protein tyrosine phosphatase dLAR and promotes the growth of presynaptic terminals (Johnson et al., 2006). Although syndecan was initially reported to be acting cell-autonomously in the motoneuron terminal, further evidence suggests that it might be provided post-synaptically by muscle cells (Nguyen et al., 2016). In mammals, the syndecan family has 4 members. Syndecan 2 is expressed in neurons of young and adult rats (Hsueh and Sheng, 1999) and progressively concentrates at asymmetric synapses in most regions of the adult rat brain 
(Hsueh et al., 1998). It can promote the formation of dendritic spines in cell culture (Ethell and Yamaguchi, 1999; Ethell et al., 2000; Lin et al., 2007), notably by facilitating FGF22 targeting to dendritic filopodia and spines (Hu et al., 2016). In turn, FGF22 promotes presynaptic differentiation hence

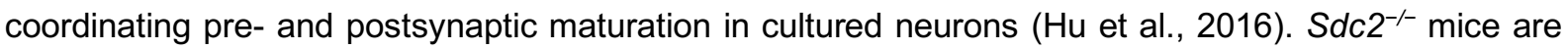
born alive with angiogenic defects, but the characterization of their nervous system has not been reported (Corti et al., 2019). Syndecan-3 is also highly expressed in the developing and adult brain, but is almost exclusively detected in axonal tracts (Hsueh and Sheng, 1999). Inactivation of Sdc3 does not cause global abnormalities of the brain anatomy (Kaksonen et al., 2002) but the laminar structure of the cerebral cortex is perturbed as a result of impaired radial migration (Hienola et al., 2006). No modification of synaptic density nor basal transmission were reported in the hippocampus. However, Sdc $3^{-/}$mice have enhanced LTP in the CA1 area and impaired hippocampus-dependent memory behaviors (Kaksonen et al., 2002), suggesting that syndecan 3 is required for synaptic plasticity.

HSPGs, in particular SDN-1, the sole syndecan member in C. elegans, is expressed and functions in the nervous system (Minniti et al., 2004; Rhiner et al., 2005). Moreover, indirect evidence suggests that heparan sulfates are highly enriched at $C$. elegans NMJs and are carried by SDN-1 (Attreed et al., 2012). We therefore used the $C$. elegans $\mathrm{NMJ}$ as a genetically-tractable model to investigate further the synaptic function of SDN-1. In C. elegans each body-wall muscle cell receives excitatory and inhibitory innervation from cholinergic and GABAergic motoneurons. The cholinergic versus GABAergic identity of postsynaptic domains is specified by the anterograde synaptic organizer Ce-Punctin/MADD-4 (PinanLucarré et al., 2014). This evolutionarily conserved ECM protein belongs to the poorly characterized ADAMTS-like proteins that contain multiple thrombospondin type-1 repeat (TSR) and immunoglobulin (Ig) domains as well as structurally-unsolved domains in common with the ADAMTS family (Apte, 2009). The functions of its vertebrate orthologs Punctin1/ADAMTSL1 and Punctin2/ADAMTSL3 are ill-defined, but Punctin2 is expressed in the brain and was identified as a susceptibility gene for schizophrenia (Dow et al., 2011).

Ce-punctin generates long (L) and short (S) isoforms by use of alternative promoters. The combination of isoforms present in the synaptic cleft controls the identity of the postsynaptic domains. Punctin $L$ is exclusively secreted by cholinergic neurons and triggers the postsynaptic localization of two types of ionotropic acetylcholine receptors (AChRs): levamisole-sensitive AChRs (L-AChRs) that are heteromeric and can be activated by the nematode-specific cholinergic agonist levamisole, and the nicotine-sensitive AChRs ( $\mathrm{N}$-AChRs) that are homomeric, activated by nicotine, and evolutionarily very close to the $\alpha 7 \mathrm{AChRs}$ expressed in the mammalian brain. L-AChRs interact with an extracellular scaffold (Gally et al., 2004; Gendrel et al., 2009; Rapti et al., 2011) to form microclusters that are positioned at synapses by Punctin L. The Punctin-dependent clustering of N-AChRs relies on a distinct uncharacterized pathway. The short isoform Punctin $S$ is secreted by both cholinergic and GABAergic motoneurons. It triggers the postsynaptic clustering of type A GABA receptors (GABAARs) at GABAergic NMJs. At cholinergic NMJs, it associates with Punctin $L$ and inhibits the inappropriate recruitment of $\mathrm{GABA}_{A} R$ s by Punctin L. Punctin $S$ controls the clustering of $\mathrm{GABA}_{A}$ Rs by two convergent molecular pathways (for review, see Zhou and Bessereau 2019). First, Punctin S binds and clusters the synaptic adhesion molecule NLG-1/neuroligin in front of GABAergic boutons, which controls the synaptic 
bioRxiv preprint doi: https://doi.org/10.1101/2020.11.25.395806; this version posted November 26, 2020. The copyright holder for this preprint (which was not certified by peer review) is the author/funder, who has granted bioRxiv a license to display the preprint in perpetuity. It is made available under aCC-BY-NC-ND 4.0 International license.

localization of $\mathrm{GABA}_{A}$ Rs (Maro et al., 2015; Tu et al., 2015). Second, it binds, recruits, and likely

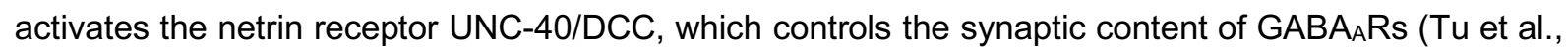
2015). UNC-40/DCC nucleates an intracellular scaffold by physically interacting with FRM-3, a FERM (p4.1, Ezrin, Radixin, Moesin) protein orthologous to FARP1/2 (Zhou et al., 2020). FRM-3 multimerizes and recruits LIN-2, the ortholog of CASK (Calcium calmodulin dependent Serine/Threonine kinase), which might provide a hub to physically connect the GABA $A_{A}$ s, FRM-3/FARP and NLG-1/neuroligin. Except NLG-1, these molecules are also present at cholinergic NMJs but their precise function was not characterized.

Here we show that SDN-1/syndecan is concentrated at NMJs by Punctin and modulates the content of receptors at excitatory and inhibitory NMJs. Remarkably, SDN-1 is the core component supporting the clustering of homomeric N-AChRs. Through physical interaction with LIN-2/CASK and FRM3/FARP, SDN-1 stabilizes a DCC-dependent synaptic scaffold at cholinergic NMJs, which in turn recruits $\mathrm{N}$-AChRs. Since all these proteins are evolutionarily conserved, these results provide a framework to analyze the molecular mechanisms controlling the localization of alpha-7 AChRs in mammalian neurons. 


\section{RESULTS}

\section{Syndecan / SDN-1 is a synaptic protein at NMJs}

Previous studies suggested that SDN-1 localizes in synapse-rich regions of the $C$. elegans nervous system based on the strong decrease of GAG immunoreactivity in the nerve ring and the nerve cords of $s d n-1$ mutants (Attreed et al., 2012). To define the precise localization of the SDN-1 HSPG, we used the CRISPR/Cas9 technique to insert the fluorescent protein mNeonGreen (mNG) at the N-terminus of SDN-1 immediately after its signal peptide (Fig. 1A). In adult worms, mNG-SDN-1 was detected at high level in the nerve ring and along the ventral and dorsal cords (Fig. 1B). The fusion protein mNG-SDN-1 appeared functional based on the absence of abnormal visible phenotypes of knock-in animals, the absence of obvious axonal outgrowth defects, and the normal content of $A C h$ and GABA receptors at NMJs as compared to the wild type (Fig. S1).

To test if mNG-SDN-1 was enriched at synapses, we used knock-in strains expressing L-AChRs or GABARs tagged with red fluorescent proteins. Visualization of the ventral and dorsal nerve cords at high magnification showed that mNG-SDN-1 was highly concentrated at NMJs. Quantitative analysis showed that both $\mathrm{ACh}$ and $\mathrm{GABA}$ A receptors almost completely colocalized with syndecan (Fig. 1C, D, $\mathrm{G})$. We also measured the SDN-1 density at each synaptic type using the presynaptic marker CLA1/Clarinet (Xuan et al., 2017), which accurately delineates active zones (Fig. 1E, F), and we observed that SDN-1 was equally abundant at excitatory and inhibitory NMJs (Fig. $1 \mathrm{H}$ ).

\section{Syndecan controls the synaptic localization of $\alpha 7$-like N-AChRs}

To identify putative functions of SDN-1 at synapses, we analyzed NMJs in sdn-1(zh20) null mutants, hereinafter referred to as $s d n-1(0)$. The number of synapses was not changed in $s d n-1(0)$ mutants as compared to the wild type, based on presynaptic markers of either cholinergic or GABAergic terminals (Fig. 2A, S2A). By contrast, we observed strong alterations of postsynaptic receptor distribution. First, the synaptic content of heteromeric L-AChRs was decreased by roughly $60 \%$ in sdn1(0) mutants as compared to the wild type, based on the fluorescence of the UNC-29-RFP subunit expressed from a knock-in allele (Fig. 2B). The remaining receptors were still clustered at synapses. Second, homomeric N-AChRs, that are homologous to $\alpha 7$ nicotinic receptors in mammals, were almost undetectable based on ACR-16-wrmScarlet fluorescence (Fig. 2C). Third, synaptic GABA $A_{A}$ content was also reduced, albeit to a lesser extent, with a roughly $35 \%$ decrease of UNC-49-RFP fluorescence at synapses (Fig. S2B). Because of the more modest impact of syndecan loss at GABAergic NMJs, we subsequently focused our analysis on SDN-1-dependent localization of AChRs.

To confirm the synaptic localization defects of AChRs in sdn-1(0) mutants, we performed electrophysiological analysis. We first evoked synaptic release of acetylcholine by optogenetic stimulation of cholinergic motoneurons. The L-AChR-dependent response recorded in the body-wall muscles of $s d n-1(0)$ mutants was diminished by $60 \%$ as compared to the wild type (Fig. 2D), while the N-AChRdependent response was reduced by $80 \%$ (Fig. 2E). Such decrease could be due to a redistribution of receptors outside of the synapses or to a decrease of functional receptors at the plasma membrane. To test these hypotheses, we pressure-applied selective agonists of L- or N-AChRs. The response to 
levamisole was similar in $s d n-1(0)$ and wild-type animals (Fig. 2F), indicating that in the absence of syndecan, L-AChRs are less efficiently clustered at synapses but remain present at the plasma membrane. By contrast, the response to nicotine was decreased by $40 \%$ in sdn-1(0) mutants (Fig. $2 \mathrm{G}$ ). This decrease was much smaller than the almost complete disappearance of $\mathrm{N}$-AChR-dependent synaptic responses. Therefore, it suggests that syndecan has two functions. First, it is necessary for clustering N-AChRs at synapses. Second, it would stabilize N-AChRs at the plasma membrane. A simple-model would predict that $\mathrm{N}$-AChRs are less stable and get endocytosed or degraded when they are not concentrated at the synapse.

Altogether, these data show that syndecan is an essential component required for proper synaptic localization of $\alpha$-like N-AChRs and, to a lesser extent, of heteromeric L-AChRs.

\section{Synaptic syndecan is mainly expressed by postsynaptic muscle cells}

NMJs are established between neurons and muscles and are in close contact with epidermal cells. We therefore wondered which tissue contributed to the expression of syndecan at synapses. Previous studies using traditional transgenic transcriptional reporters showed that $s d n-1$ was expressed in epidermis and neurons, but not in body-wall muscle cells (Minniti et al., 2004; Rhiner et al., 2005). To further investigate the $s d n-1$ expression pattern, we engineered a chromosomal bi-cistronic reporter by inserting an SL2-wrmScarlet cassette in the sdn-1 locus after the end of its coding sequence (Fig. 1A). In agreement with previous results, red fluorescence was detected in most tissues including epidermis and motoneurons, but wrmScarlet was also readily detected in body-wall muscles (Fig. 3A).

To identify the source of synaptic syndecan, we performed tissue-specific degradation of SDN-1. Using CRISPR/Cas9, we fused an Auxin-Inducible-Degron (Zhang et al., 2015) linked to mNG at the SDN-1 C-terminus (Fig. 1A) and we expressed the TIR-1 E3-ligase under various tissue-specific promoters (Fig. S3). SDN-1-AID-mNG properly localized at synapses (Fig. 3B), similarly to mNG-SDN1 (Fig. 1C). Ubiquitous expression of TIR-1 caused a complete disappearance of fluorescence in the worm, including at the nerve cords (Fig. 3B, C). Specific degradation of SDN-1-AID-mNG either in the epidermis (Fig. 3B, D) or in neurons (Fig. 3B, E) roughly caused a $25 \%$ reduction of the synaptic fluorescence, while degradation of SDN-1-AID-mNG in body-wall muscles caused a $70 \%$ decrease of the synaptic fluorescence (Fig. 3B, F). Therefore, these data indicate that SDN-1 is expressed by pre-, post- and peri-synaptic cells, yet the vast majority of SDN-1 present at synaptic sites is contributed by postsynaptic muscle cells.

\section{Ce-Punctin/MADD-4 localizes syndecan at synapses}

AChR localization at cholinergic NMJs is controlled by the anterograde synaptic organizer CePunctin/MADD-4 (Pinan-Lucarré et al., 2014). madd-4 encodes madd-4L (long) and madd-4B (short) isoforms (Fig. 4A). The long isoforms MADD-4-L are present at cholinergic NMJs and in their absence, both $\mathrm{L}-$ and $\mathrm{N}-\mathrm{AChR}$ clusters are redistributed and no longer retained at synapses. The short isoform

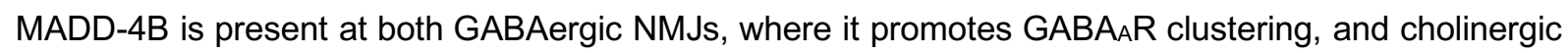
NMJs, where it prevents MADD-4L from recruiting GABA $A_{A}$ Rs (Pinan-Lucarré et al., 2014). To test if MADD-4 could also regulate the localization of SDN-1, we introduced the $m N G$-sdn-1 knock-in allele in 
various madd-4 mutant backgrounds. mNG-SDN-1 was almost undetectable at the nerve cords of madd-4(0) null mutants (Fig. 4B). We then wondered if both madd-4 isoforms were required for SDN-1 localization. madd- $4 L$ mutation had no impact on the localization of SDN-1, measured by the correlation with a marker of presynaptic GABA boutons, nor on its expression level (Fig. 4C-E). Genetic removal of MADD-4B changed the localization of mNG-SDN-1 without affecting its overall content at the nerve cord. Based on the labelling of GABAergic terminals, mNG-SDN-1 was no longer detectable at GABAergic synapses but relocalized at cholinergic junctions, where MADD-4L was still present (Fig. 4C-E). Hence, these results show that SDN-1 localization is controlled by the neurally-secreted extracellular matrix protein MADD-4, and that this localization can be instructed by any of the two MADD-4 isoforms.

We then asked whether the negatively charged GAG chains of SDN-1 might be required for its localization, because MADD-4 is predicted to contain a highly positively-charged immunoglobulin domain critical for its function (Maro et al., 2015; Seetharaman et al., 2011). SDN-1 contains two putative heparan sulfate attachment sites in its $\mathrm{N}$-terminal region, and a third putative site closer to the transmembrane region (Minniti et al., 2004). We mutated these sites by CRISPR/Cas9 in a C-terminally tagged version of SDN-1, in order to avoid any potential interaction of the fluorescent protein with the extracellular matrix (Fig. S4A). Immunoprecipitation followed by western blot analysis first indicated that SDN-1 likely form SDS-resistant dimers, as previously described for mammalian syndecans (Fig. S4B) (Asundi and Carey, 1995; Choi et al., 2005). Because we did not detect any additional weight difference between the double and triple mutants, the third putative GAG attachment site is most probably not modified (Fig. S4B). Consistently, when SDN-1-mNG precipitates were treated with heparinase (I and III) the apparent molecular weight was similar as SDN-1( 2 GAG)-mNG (Fig. S4B). Noteworthy, SDN$1(\triangle 3 G A G)$-mNG still localized at synapses, but the overall fluorescence level was decreased by $30 \%$ when removing one chain, and by about $50 \%$ when mutating the remaining sites as compared to the wild-type SDN-1-mNG (Fig. S4C). This suggests that GAG chains stabilize SDN-1 at the synapse, maybe through direct interaction with MADD-4, yet it is not possible to completely rule out that the point mutations introduced in the extracellular domain slightly impair the expression or the trafficking of the protein. In any case, these results demonstrate that the SDN-1 core protein carries sufficient information for MADD-4 dependent synaptic localization.

Since MADD-4 functionally interacts with SDN-1, we asked whether SDN-1 would be able, in turn, to regulate MADD-4. In $s d n-1(0)$ mutants, the synaptic content of MADD-4 was reduced by $30 \%$ as compared to the wild type, based on the expression of an RFP C-terminal fusion that labels both MADD4 isoforms (Fig. 4F). In $s d n-1$ ( $\triangle 3 G A G$ )-mNG, MADD-4-RFP was also decreased, albeit to a lesser extent (17\%) (Fig. 4G). The interpretation of these results is complicated because removing the GAG chains causes a partial loss of SDN-1, yet they suggest that the GAG chains carried by SDN-1 might partly stabilize MADD-4 at the synapse.

\section{SDN-1 recruits LIN-2/CASK at cholinergic synapses to cluster $\alpha 7$-like N-AChRs}

SDN-1 contains in its C-terminus an evolutionarily conserved PDZ domain binding site, which was demonstrated to interact with the scaffolding protein CASK in the mammalian nervous system (Ethell and Yamaguchi, 1999; Hsueh et al., 1998). To determine whether the clustering of $\alpha 7$-like N-AChRs by 
SDN-1 is dependent on a SDN-1/LIN-2CASK interaction, we used CRISPR/Cas9 to delete the last 4 residues of $\mathrm{mNG}-\mathrm{SDN}-1$. The resulting protein, $\mathrm{mNG}-\mathrm{SDN}-1(\triangle \mathrm{EFYA})$, properly localized at synapses, although synaptic content was slightly decreased (Fig. S5A). However, the deletion of the PDZ-binding site caused an almost complete disappearance of $\mathrm{N}$-AChRs from the nerve cord, while L-AChRs remained expressed and clustered at synapses indistinguishably from the wild type (Fig. 5A, B). We previously demonstrated that LIN-2, the ortholog of CASK that contains a PDZ domain, was present at GABAergic NMJs where it promotes the recruitment of $G_{A B A} A_{A}$ at GABAergic synapses (Zhou et al., 2020). Over the course of that study, we also showed that LIN-2/CASK was additionally present at cholinergic synapses. We therefore hypothesized that LIN-2 might be involved in N-AChR clustering by interacting with the SDN-1 PDZ binding site. Analysis of acr-16-wrmScarlet in lin-2(0) null mutants revealed a $95 \%$ reduction of N-AChR content at the nerve cord (Fig. 5C). This defect could be rescued by muscle specific expression of either the long isoform LIN-2A or the short isoform LIN-2B that lacks the N-terminal CaM-kinase domain but retains the PDZ, SH3 and the MAGUK domains (Fig. 5C).

We then tested if LIN-2/CASK and SDN-1 were able to physically interact. Using in vitro translated and purified recombinant proteins, we showed that the intracellular domain of SDN-1 could efficiently pull-down LIN-2B. This interaction was strongly reduced after deletion of the SDN-1 PDZ-binding site (Fig. 5D). The relevance of this interaction was further tested in vivo. Analysis of LIN-2 expression in $s d n-1(0)$ null mutants revealed a $55 \%$ decrease of LIN-2 at the nerve cord (Fig. 5E, G). Strikingly, the remaining LIN-2 was exclusively detected at GABAergic synapses, as shown by its perfect colocalization with neuroligin/NLG-1 that is only present at GABA synapses (Maro et al., 2015; Tu et al., 2015) (Fig. 5E-G). These results suggest that SDN-1 is the limiting factor that controls the localization of LIN-2 at cholinergic synapses. By contrast, SDN-1 was only decreased by $16 \%$ in lin-2(0) (Fig. 5H). Next, we analyzed the distribution of LIN-2 in $m N G-s d n-1$ ( $\triangle E F Y A$ ) mutants. LIN-2 no longer completely colocalized with truncated SDN-1 (Fig. 5I, J) and was mostly present at GABAergic NMJs (Fig. S5B-D). Because we showed that neuroligin is necessary for LIN-2 localization at GABAergic synapses, this predicts that removing both syndecan and neuroligin would cause a complete loss of LIN-2 at NMJs. Accordingly, LIN-2-GFP was almost undetectable at the nerve cord of $n l g-1(0)$; sdn-1(0) double mutants (Fig. 5K).

Altogether, these data indicate that LIN-2/CASK is positioned in the postsynaptic domains of excitatory and inhibitory synapses by two distinct transmembrane proteins, namely syndecan SDN-1 and neuroligin NLG-1, respectively, that each provide binding sites for LIN-2, which in turn participates to the recruitment of $A C h R$ and $G A B A_{A} R$.

\section{FRM-3/FARP bridges $\alpha 7$-like N-AChRs with LIN-2/CASK and SDN-1}

In a previous study, we showed that FRM-3, a FERM protein orthologous to mammalian FARP1/2, interacts with LIN-2 at GABAergic synapses to promote the recruitment of GABA receptors by neuroligin (Zhou et al., 2020). Because FRM-3 was also detected at cholinergic NMJs, we tested if FRM-3 might also be involved in the clustering of N-AChR. Strikingly, ACR-16-wrmScarlet was almost undetectable in a frm-3(0) null mutant (Fig. 6A). This phenotype could be rescued by muscle-specific expression of either full length FRM-3A or by its FERM-FA (FERM adjacent) domain. Interestingly, the FERM domain 
alone was not able to rescue N-AChR clustering defects, as also observed for GABA receptors at inhibitory NMJs (Fig. 6A) (Zhou et al., 2020).

We then analyzed the expression of FRM-3-GFP in a $s d n-1(0)$ mutant and observed a $55 \%$ decrease of fluorescence at the nerve cord (Fig. 6B, C). However, in contrast to LIN-2, the FRM-3 pattern remained unchanged (Fig. 6B-D). This result is consistent with our previous analysis showing that FRM-3 level was unaffected by the loss of LIN-2 (Zhou et al., 2020). Hence, despite the loss of LIN2 at cholinergic synapses in sdn-1(0) mutants, FRM-3 remained partially concentrated in postsynaptic domains of cholinergic synapses. In a reciprocal experiment, we measured a $47 \%$ decrease of SDN-1 content at the nerve cord of frm-3(0) mutants (Fig. 6E), much more than in lin-2(0) mutants. This suggested that the interactions between FRM-3 and LIN-2 with SDN-1 were different. Indeed, in contrast with LIN-2, FRM-3 content was not reduced by deleting the PDZ-binding site of SDN-1 (Fig. 6F). Interestingly, an early study reported direct binding of the FERM domain of Ezrin and Syndecan-2 through the DEGS motif present in its $C 1$ intracellular region (Granés et al., 2003). This sequence is conserved in SDN-1 (Fig. 1A). CRISPR/Cas9 deletion of the KKDEGS sequence caused a $50 \%$ decrease of mNG-SDN-1 fluorescence level, similar to what was observed in frm-3(0) mutants (Fig. 6E). The deletion of the SDN-1 KKDEGS sequence in frm-3(0) animals did not cause an additional decrease of mNG-SDN-1 fluorescence, suggesting that the $\mathrm{C} 1$ domain of SDN-1 is the site of interaction with FRM-3. We then looked at the effect of SDN-1 KKDEGS sequence deletion on N-AChRs: ACR-16wrmScarlet fluorescence was almost undetectable in these mutants, as observed in frm-3(0) mutants (Fig. 6G). Therefore, FRM-3 might recruit or stabilize SDN-1 at synaptic sites through a FERM-FA/C1 interaction, which in turn recruits ACR-16.

Our data show that FRM-3 and LIN-2 are both critical for SDN-1 dependent clustering of N-AChR at cholinergic synapses. We and others previously identified physical interactions between FRM-3 and LIN-2 (Tong et al., 2015; Zhou et al., 2020). We therefore hypothesized that they might form a complex connecting N-AChR and SDN-1. Accordingly, in vitro pull-down experiments demonstrated interactions of the FERM-FA domain of FRM-3 with the intracellular domain of SDN-1 and with the TM3-TM4 cytoplasmic loop of ACR-16 (Fig. 6H, I). Some interaction could also be detected between LIN-2B and ACR-16 (Fig. 6I). Altogether, these data suggest that a macromolecular scaffold assembles at cholinergic synapses to localize N-AChR.

\section{Ce-Punctin/MADD-4 recruits UNC-40/DCC and syndecan/SDN-1 to stabilize the $\alpha 7$-like N-AChR ACR-16 at the synapse}

We previously showed that FRM-3 is localized at NMJs by the UNC-40/DCC transmembrane receptor through a physical interaction between its FERM-FA domain and the C-terminal P3 domain of UNC-40 (Zhou et al., 2020). We therefore asked whether UNC-40 might also be required for the synaptic localization of N-AChRs. In unc-40(0) mutants, ACR-16-wrmScarlet fluorescence was indeed reduced by roughly $70 \%$, but the remaining signal had a punctate pattern (Fig. 7A). Interestingly, just deleting the $\mathrm{P} 3$ domain of UNC-40 caused similar loss of ACR-16, while unc-4O( $\triangle P 3)$ mutants are much healthier and have less axonal and cell migrations defects than unc-40(0) animals (Zhou et al., 2020). 
Since we know that P3 interacts with FRM- 3 and that the loss of FRM- 3 causes a decrease of SDN1 , we predict that UNC-40 might stabilize SDN-1 via FRM-3. We therefore analyzed the SDN-1 content at the nerve cord of unc-40(0) mutants. In agreement with our hypothesis, we observed a $50 \%$ loss of mNG-SDN-1 fluorescence (Fig. 7B). However, these data do not rule out a reverse interaction between SDN-1 and UNC-40, as previously reported in other systems (Bennett et al., 1997; Blanchette et al., 2015). We therefore analyzed the synaptic localization of RFP-UNC-40 in sdn-1(0) mutants and observed a $38 \%$ decrease of fluorescence (Fig. 7C). Interestingly, UNC-40 remained present at both cholinergic and GABAergic NMJs (Fig. 7D, E). These results support the existence of a crosstalk between SDN-1 and UNC-40 to maintain the proper level of either protein at NMJs.

We and others previously demonstrated that UNC-40 localization at NMJs depends on the synaptic organizer MADD-4 ( Tu et al., 2015). Since we demonstrated that MADD-4 localizes SDN-1 at synapses, UNC-40 localization might depend on an interaction with SDN-1. Alternatively, MADD-4 is able to bind UNC-40 in heterologous systems (Tu et al., 2015) and might therefore localize UNC-40 at synapses independently from SDN-1. To test these hypotheses, we analyzed RFP-UNC-40 expression in madd4(0) mutants and observed a stronger decrease than in sdn-1(0) mutants (Fig. 7C), suggesting that MADD-4 has the capability to localize UNC-40 at NMJs independently from SDN-1. Consistently, the loss of UNC-40 was not aggravated in a madd-4(0); sdn-1(0) double mutant as compared to madd-4(0) single mutants (Fig. 7C).

Altogether, these results show that MADD-4 is the key-molecule that recruits SDN-1 and UNC-40 at NMJs. These three molecules then engage a network of extracellular and intracellular interactions, involving FRM-3 and LIN-2, to stabilize each other at the synapse. This, in turn, supports the recruitment of ACR-16 at cholinergic synapses.

\section{The intracellular domain of SDN-1 is sufficient to relocalize N-AChRs at GABAergic synapses independently from L-AChRs}

At cholinergic synapses, SDN-1 is required for the localization of both L-AChRs and N-AChRs. Yet, the interaction between the intracellular C-terminus of SDN-1 and the PDZ domain of LIN-2/CASK is specifically involved in the clustering of homomeric N-AChRs, but not heteromeric L-AChRs. We therefore asked whether SDN-1 was only a permissive factor requiring additional components to specify $\mathrm{N}$-AChR localization or if it was carrying sufficient information to dictate the receptor localization. To answer this question, we expressed in the muscle cells of $s d n-1(0)$ mutants a GFP-tagged chimeric protein containing the extracellular and transmembrane regions of NLG-1 and the intracellular domain of SDN-1 (Fig. 8A). As predicted, this NLG-1 chimera is localized at GABA synapses (Fig. 8B) through MADD-4 interactions (Tu et al., 2015). Strikingly, as opposed to sdn-1(0) mutants, ACR-16-wrmScarlet were readily detected in transgenic animals but they relocalized at GABAergic NMJs together with the chimera (Fig. 8C, D). In contrast, the decreased level of L-AChRs was not rescued and the remaining receptors remained localized at cholinergic synapses (Fig. $8 \mathrm{E}-\mathrm{G}$ ). These results demonstrate that the intracellular domain of SDN-1 is necessary and sufficient to recruit N-AChR at synaptic sites. Furthermore, despite the fact that homomeric and heteromeric AChRs colocalize at cholinergic NMJs, their clustering relies on 2 distinct machineries (see Fig. 9 and discussion). 


\section{DISCUSSION}

Our results identified the syndecan SDN-1 as a core organizer of cholinergic and, to a lesser extent, GABAergic neuromuscular junctions in $C$. elegans. SDN-1 is absolutely required for the synaptic clustering of homomeric alpha-7-like N-AChRs and is necessary for achieving proper synaptic levels of heteromeric L-AChRs and GABAA receptors. We therefore uncovered a previously unknown function of syndecan in the direct control of synaptic receptor composition. Syndecan acts by bridging extracellular matrix components with intracellular scaffolding proteins (Fig. 9). In our model, the anterograde organizer Punctin is secreted by cholinergic and GABAergic motoneurons in the synaptic cleft and triggers appropriate postsynaptic differentiation. At cholinergic synapses, Punctin has at least two parallel functions. First, it localizes the syndecan produced by muscle cells at postsynaptic sites. Second, it concentrates and likely activates the transmembrane receptor UNC-40/DCC. Syndecan, in turn, stabilizes Punctin and UNC-40 at synaptic sites. Coincident clustering of SDN-1 and UNC-40 at cholinergic NMJs triggers the intracellular recruitment of the scaffolding molecules LIN-2/CASK and FRM-3/FARP by direct interaction with the PDZ domain binding site of SDN-1 and the C-terminal P3 domain of UNC-40, respectively. The FERM-FA domain of FRM-3/FARP also engages direct interaction with SDN-1, likely with its submembrane $\mathrm{C} 1$ domain. The resulting CASK/FARP complex then triggers synaptic clustering of the $\alpha 7$-like N-AChR.

\section{The synaptic localization of syndecan is controlled by the ECM protein Punctin}

The molecular mechanisms that control the synaptic localization of syndecan at drosophila NMJs or in rodent synapses remain elusive. At the $C$. elegans $\mathrm{NMJ}$, our genetic analyses demonstrate the instructive role of Punctin for synaptic localization of SDN-1/syndecan. Strikingly, removing Punctin from GABAergic NMJs relocalizes SDN-1 exclusively at cholinergic NMJs, while in a Punctin null mutant, SDN-1 is almost undetectable. The most parsimonious model would predict a direct interaction between the C-terminal half of Punctin, shared by all Punctin isoforms, and SDN-1, adding to the long list of extracellular matrix syndecan interactors (see Gondelaud and Ricard-Blum, 2019). This interaction must involve the SDN-1 core protein since $\triangle \mathrm{GAG}$ SDN-1 mutants still localize at the synapse. Accordingly, the human ADAMTS1 protease, which contain structural domains similar to Punctin, is able to cleave syndecan-4 independently of the presence of GAG chains (Rodríguez-Manzaneque et al., 2009). However, heparan sulfate (HS) chains are important to regulate the amount of SDN-1 at synapses. Using CRISPR/Cas9 in combination with biochemical analysis, we demonstrated that SDN-1 carries two HS chains. Mutation of the GAG-attachment sites causes a $50 \%$ decrease of synaptic SDN-1. HS form long, highly negatively charged molecules able to engage versatile electrostatic interactions (Xu and Esko, 2014). Punctin is a good candidate for synaptic stabilization of SDN-1 through HS interaction because it contains an Ig-like domain with a predicted highly electropositive surface pocket, which we demonstrated to bind heparin in vitro (Platsaki et al, submitted). Conversely, SDN-1 tunes the synaptic amount of Punctin since we observed a $30 \%$ decrease of Punctin in sdn-1 null mutants. The synaptic localization mechanisms of Punctin are unknown and SDN-1 is the first identified component able to regulate the amount of Punctin. Although Punctin might interact with a yet unidentified presynaptic 
receptor, an alternative hypothesis is that it is locally deposited at synaptic terminals and its interaction with the ECM would prevent its diffusion and trigger the local differentiation of postsynaptic domains (Pinan-Lucarré et al., 2014). The Punctin/SDN-1 crosstalk identified at the C. elegans NMJ provides an interesting example of a positive feedback loop between the synaptic organizer and its effector, which likely contributes to synaptic stabilization.

\section{Cooperation between syndecan- and UNC-40/DCC-dependent signaling to localize $\alpha 7$-like $\mathrm{N}$ - AChRs.}

Syndecan impacts the second signaling output of Punctin, which involves the transmembrane receptor UNC-40/DCC. During development, Punctin promotes the growth of muscle arms toward nerve cords by positioning and activating UNC-40-dependent remodeling of the actin cytoskeleton (Alexander et al., 2009). In the adult, Punctin binds, recruits and likely activates UNC-40/DCC at the postsynaptic sites of NMJs (Tu et al., 2015). At GABAergic NMJs, UNC-40 activation sets the amount of synaptic $\mathrm{GABA}_{A}$ Rs by promoting their recruitment to NLG-1/neuroligin (Zhou et al., 2020). Here we show that in addition to its dependence on Punctin, the synaptic content of UNC-40 is regulated by SDN-1, both at cholinergic and GABAergic synapses. In the absence of syndecan, UNC-40 remains synaptic but its content is reduced by half. DCC is known to bind heparin (Bennett et al., 1997) and numerous studies have documented the interplay between GAGs and DCC-signaling for cellular and axonal migration in various organisms (Blanchette et al., 2015, 2017; Bülow et al., 2002; Matsumoto et al., 2007; Rhiner et al., 2005). An elegant structural study demonstrated the critical role of HS for the binding of netrin to DCC by providing sulfate ions that bridge the positively charged surfaces that interact which each other on both proteins (Finci et al., 2014). Similarly, syndecan might serve as a co-receptor to strengthen the binding of Punctin on UNC-40, or stabilize UNC-40 at the plasma membrane by favoring its interaction with the ECM.

Syndecan- and DCC-dependent signaling then converge to build an intracellular scaffold containing FRM-3/FARP and LIN-2/CASK at cholinergic NMJs. This configuration parallels what we recently described at GABAergic NMJs for the adhesion molecule NLG-1/neuroligin and the transmembrane receptor UNC-40/DCC where (i) UNC-40 recruits FRM-3 through its C-terminal P3 domain, (ii) FRM-3 recruits LIN-2, (iii) LIN-2 binds the C-terminal tail of neuroligin through its PDZ domain, (iv) LIN-2 interacts with the intracellular loop of the GABAAR UNC-49 and stabilizes the receptors at the synapse by bridging them with Punctin-dependent neuroligin clusters (Zhou et al., 2020). At a first glance, syndecan replaces neuroligin at cholinergic synapses to eventually achieve N-AChR clustering. We demonstrated that SDN-1 interacts with LIN-2/CASK via its C-terminal PDZ domain binding site and this interaction is necessary for the localization of LIN-2/CASK at cholinergic synapses. The PDZ-dependent interaction between syndecans and CASK is evolutionarily conserved (Hsueh et al., 1998). Interestingly, CASK is mostly found in axons during development, together with SDC3, and redistributes to somatodendritic compartments in the adult, where it is coenriched with SDC2 at various synapses (Hsueh and Sheng, 1999; Hsueh et al., 1998). Our results in C. elegans suggest that syndecan localization might instruct the subcellular localization of CASK in mammalian neurons. As FRM-3 stabilizes SDN-1, the formation of the UNC-40-dependent intracellular scaffold potentially provides an 
additional level for locally-restricted stabilization of postsynaptic specialization in addition to the positive crosstalk between Punctin and syndecan in the synaptic cleft.

\section{The $\alpha 7$-like N-AChR clustering relies on an evolutionarily-conserved intracellular scaffold}

The intracellular moiety of SDN-1 syndecan is the core component that controls the synaptic clustering of the alpha-7-like N-AChR by nucleating the assembly of the LIN-2/CASK-FRM-3/FARP complex at cholinergic synapses. Punctin therefore achieves N-AChR clustering by positioning SDN-1 in register with cholinergic boutons, and SDN-1 subsequently recruits N-AChRs via the LIN-2/FRM-3 cytoplasmic complex. This mechanism is fundamentally different from the clustering mechanisms of the heteromeric L-AChRs, where a set of extracellular proteins associate with L-AChRs to form small clusters that are subsequently localized at cholinergic synapses by the long isoform of Punctin (Gally et al., 2004; Gendrel et al., 2009; Pinan-Lucarré et al., 2014; Rapti et al., 2011). However, even if SDN-1 is not strictly required for L-AChR clustering, it modulates the synaptic content of L-AChRs. This likely involves extracellular interactions because neither the disruption of the SDN-1/LIN-2 association nor the ectopic expression of the SDN-1 intracellular domain has an impact on L-AChRs. The L-AChR decrease observed in $s d n-1(0)$ mutants might be a consequence of the reduced amount of Punctin present at synapses. SDN-1 might also stabilize the interactions between Punctin-long and the L-AChR clustering machinery, which have still not been solved at the molecular level.

The central and specific role of SDN-1 for N-AChR clustering at cholinergic synapses raises questions about the mechanisms underlying this specificity. Not only SDN-1 is equally present at GABAergic NMJs, but its associated proteins UNC-40/DCC, FRM-3/FARP and LIN-2/CASK are used at inhibitory NMJs to cluster GABAARs. Yet, ACR-16 is exclusively detected at cholinergic synapses. Several scenarios can be envisioned. First, NLG-1 could outcompete SDN-1 for LIN-2/CASK binding and stabilize a complex that preferentially binds GABAARs. Second, Punctin-long, which is exclusively present at cholinergic NMJs, might induce distinct oligomerization of SDN-1 at the nanoscale, which is known to impact its signaling output (Choi et al., 2005; Jang et al., 2018). Third, SDN-1 might be differentially phosphorylated (Ethell et al., 2001; Horowitz and Simons, 1998; Murakami et al., 2002; Oh et al., 1997), modified or associated with a non-identified cofactor to promote the formation of an ACR16 binding complex. The insertion of ACR-16 in the muscle membrane was shown to be regulated by Wnt ligands possibly secreted from cholinergic neurons (Babu et al., 2011; Francis et al., 2005; Jensen et al., 2012; Tikiyani et al., 2018). A crosstalk between Wnts and syndecan has been documented in many systems (Pataki et al., 2015) and SDN-1 might serve as a co-receptor at cholinergic NMJs. Yet, this putative Wnt-syndecan crosstalk can be bypassed by overexpression of the SDN-1 tail at GABAergic synapses, which emphasizes the central role of SDN-1 as a postsynaptic organizer.

The C. elegans ACR-16 is strikingly close to the mammalian $\alpha 7 \mathrm{nAChR}$ subunit (Ballivet et al., 1996; Touroutine et al., 2005). These receptors have attracted attention because they have been implicated in cognition and memory and their dysfunction was associated to neuropsychiatric disorders (for review see (Dineley et al., 2015)). In some instances, they mediate fast synaptic transmission, as in the hippocampus (Alkondon et al., 1997; Bürli et al., 2010; Fabian-Fine et al., 2001; Gray et al., 1996; Wonnacott, 1997; Zarei et al., 1999), and in many cases, they localize presynaptically on GABAergic or 
glutamatergic terminals where they enhance neurotransmitter release (Alkondon et al., 1997; Bürli et al., 2010; Fabian-Fine et al., 2001; Gray et al., 1996; Liu et al., 2001). Surprisingly, the mechanisms that regulate these localizations remain mostly unknown. Miscellaneous factors including synaptic activity, neurotrophin, anti-apoptotic Bcl-2 proteins, src kinase, Wnt and Neuroregulin signaling pathways or plasma membrane lipid composition (Brusés et al., 2001; Charpantier et al., 2005; Dawe et al., 2019; Farías et al., 2007; Hancock et al., 2008; Kawai et al., 2002), have been shown to impact $\alpha 7$ receptor localization, but always partially. Colocalization between $\alpha 7$ receptor clusters and PSD-95 family members has been described, yet direct interaction was not documented (Conroy et al., 2003; Farías et al., 2007; Parker et al., 2004). Because all the components we identified in C. elegans are evolutionarily conserved and expressed in neurons, it would be worth testing if syndecan regulates the localization of nicotinic receptors in the mammalian brain.

\section{ACKNOWLEDGEMENTS}

We thank the Hobert laboratory for plasmids, the Caenorhabditis Genetic Center (which is funded by NIH Office of Research Infrastructure Programs, P40 OD010440) for strains, Alexis Weinreb for image analysis scripts, the CIQLE Imaging facility for support and access to equipment. This work was supported by the European Research Council (ERC_Adg C.NAPSE \#695295), within the framework of the LABEX CORTEX (ANR-11-LABX-0042) of Universite de Lyon, within the program "Investissements d'Avenir" (ANR-11-IDEX-0007) operated by the French National Research Agency (ANR). Work in the Bülow lab is supported by a grant from the National Institute of Neurological Disorders and Stroke (R21NS111145).

\section{AUTHOR CONTRIBUTION}

Conceptualization, J.L.B., X.Z., C.V., H.E.B.; Methodology, X.Z., C.V., M.C., O.R., M.J.; Investigation, X.Z., C.V., M.C., O.R., M.J.; Writing -Original Draft, J.L.B, Z.X., C.V., M.J.; Writing -Review \& Editing, M.C., H.E.B.; Funding Acquisition, J.LB., H.E.B..

\section{DECLARATION OF INTERESTS}

The authors declare no competing interests. 


\section{FIGURE LEGENDS}

\section{Figure 1. SDN-1 is enriched at NMJs.}

(A) Predicted structure of SDN-1 protein (288 aa) and sdn-1 locus (2921 bp). SP (signal peptide, dark blue box), TM (transmembrane, light green box), C1 (containing the DEGS ezrin binding motif, light blue box), C2 (containing the PDZ binding motif EFYA, orange box). Three putative sites (black arrowheads) for GAG anchoring have been predicted on serine 71, 86 and 214. Open arrowheads indicate the knock-

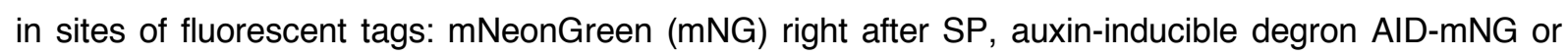
SL2-wrmScarlet (wormScarlet) right after the PDZ binding motif. The gray bar marks the location of the 1258 bp deletion in sdn-1(zh20) mutant allele. Boxes represent exons.

(B) High expression level of mNG-SDN-1 is detected in the nerve ring and along the nerve cords. Scale bar is $10 \mu \mathrm{m}$.

(C-D) Confocal detection and fluorescence profiles along the dorsal cord of mNG-SDN-1 and TagRFP$T$ labeled receptors: L-AChR is labelled from the unc-29-TagRFP-T knock-in allele and $G_{A B A} R$ is labelled from the TagRFP-T-unc-49 knock-in allele.

(E-F) Confocal detection and fluorescence profiles along the dorsal cord of mNG-SDN-1 and the presynaptic active zone marker CLA-1-BFP under the control of the cholinergic neuron-specific promoter Punc-17 (F) or under the control of the GABAergic neuron-specific promoter Punc-47 (G).

(G) Percentage of L-AChRs and GABA ${ }_{A}$ Rs colocalized with mNG-SDN-1, calculated using Manders coefficient on processed images (see material and methods for details).

(H) Mean intensity of the mNG-SDN-1 signal specifically colocalized with cholinergic or GABAergic synapses, calculated following image processing (see material and methods for details).

In this figure and all the other confocal figures, pictures are sums of Z-stacks acquired along the dorsal nerve cord by a spinning disk confocal microscope; anterior is to the left, scale bar is $10 \mu \mathrm{m}$.

In this figure and all the other figures, data distribution in each group is presented as violin plots showing lower and upper quartiles (dotted lines), median (blue line). The number of animals analyzed is indicated on the violin plots. Mann-Whitney test, n.s: not significant. See also Fig. S1.

\section{Figure 2. SDN-1 controls AChR synaptic content.}

(A) Confocal detection of the presynaptic active zone marker CLA-1-BFP under the control of the cholinergic neuron-specific promoter Punc-17 in control and $s d n-1(0)$ mutant animals. Right: quantification of active zone numbers per $50 \mu \mathrm{m}$ of dorsal cord in control and $s d n-1(0)$ animals.

(B) Confocal detection and quantification of RFP-labeled L-AChR (UNC-29-RFP) fluorescence in control and $s d n-1(0)$ animals. The fire image ruler represents the min to max value of intensities for each image. (C) Confocal detection and quantification of RFP-labeled N-AChR (ACR-16-wrmScarlet) fluorescence expressed from an acr-16-wrmScarlet knock-in allele in control and sdn-1(0) animals.

(D-E) Representative traces of isolated L-AChR (D) or total AChR (E) peak currents evoked by $10 \mathrm{~ms}$ light stimulation recorded from muscle cells of control and $s d n-1(0)$ animals expressing ChR2 in 
cholinergic motoneurons. Arrowheads mark the light stimulation onset. Isolation of L-AChR current was obtained by adding dihydro-beta-erythroidine (DH $\beta E, 0.01 \mathrm{mM})$ in the bath solution. Quantification was done for isolated L-AChR and N-AChR currents as detailed in materials and methods.

(F-G) Representative traces and quantification of peak currents evoked by $0.1 \mathrm{mM}$ levamisole $(\mathrm{F})$ or nicotine $(\mathrm{G})$ on muscle cells from control and $s d n-1(0)$ mutant animals. Arrowheads mark the $100 \mathrm{~ms}$ application onsets. Individual values are shown as dots in ( $E$ and $F$ ).

Scale bars $=10 \mu \mathrm{m}$ for (A, B and C). For the quantification of fluorescence levels, data were normalized to the mean value of the control group. Violin plots as in figure 1G. Mann-Whitney tests, ${ }^{* *} p<0.001$, ${ }^{* *} p<0.01$, n.s: not significant. See also Fig. S2.

Figure 3. Synaptic syndecan is mainly provided by postsynaptic muscle cells.

(A) Confocal detection of the transcriptional reporter SDN-1-SL2-wrmScarlet from a knock-in allele showing sdn-1 expression in epidermis, muscle cells and nerve cord. Arrowheads indicate cell bodies of motor neuron. Dotted lines delineate borders of body-wall muscle cells.

(B) Confocal detection of SDN-1-AID-mNG from dorsal nerve cord in animals grown on regular NGM plates (control) or on plates containing auxin to induce SDN-1 depletion in all tissues (animals expressing Peft-3::tir-1-bfp), in epidermis (animals expressing Pdpy-7::tir-1-bfp), in neurons (animals expressing Prab-3::tir-1-bfp), or in body-wall muscles (animals expressing Pmyo-3::tir-1-bfp).

(C-F) Quantification of SDN-1-AID-mNG fluorescence levels when SDN-1 was depleted in all tissues $(C)$, body-wall muscles $(D)$, neurons $(E)$ or epidermis $(F)$. Fluorescence levels were normalized to the mean of control.

Scale bars $=10 \mu \mathrm{m}$. Violin plots as in figure $1 \mathrm{G}$. Mann-Whitney tests, ${ }^{* * *} \mathrm{p}<0.001,{ }^{* *} \mathrm{p}<0.01$. See also Fig. S3.

Figure 4. Ce-Punctin/MADD-4 localizes SDN-1 at synapses.

(A) Structure of the madd-4 locus. Boxes represent exons. Arrows indicate the beginning of the open reading frame of madd- $4 L$ (black) and madd- $4 B$ (grey). Localization of mutations are indicated: madd4(ttTi103747) specifically disrupts the long isoform and is referred to as madd-4L(0); madd-4(tr185) specifically disrupts the short isoform and is referred to as madd-4B(0); madd-4(kr270) is a full deletion of the locus, disrupting all isoforms, and is referred to as madd-4(0).

(B) Confocal detection of mNG-SDN-1 and the presynaptic active zone marker CLA-1-BFP under the control of the cholinergic neuron-specific promoter Punc-17 in control and madd-4(0) null animals. mNGSDN-1 was barely detectable in madd-4(0) mutants.

(C) Confocal detection of mNG-SDN-1 and the presynaptic marker SNB-1-BFP under the control of the GABAergic neuron-specific promoter Punc-47 in control animals and in madd-4L(0) and madd- $4 B(0)$ mutants. The fluorescence profiles indicate $\mathrm{mNG}-\mathrm{SDN}-1$ and SNB-1-BFP fluorescence intensities along the nerve cord from the pictures above. 
(D) Quantification of mNG-SDN-1 fluorescence levels in control and madd-4L(0) and madd-4B(0) mutants. mNG-SDN-1 fluorescence intensities were normalized to the mean of control group. One-way ANOVA followed by Tukey's multiple comparison tests. " $p<0.05$, n.s: not significant.

(E) Pearson's correlation coefficient between mNG-SDN-1 and GABA presynaptic SNB-1-BFP in control and madd- $4 L(O)$ and madd- $4 B(0)$ mutants. Kruskal-Wallis test followed by Dunn's post-hoc test. ${ }^{* *} \mathrm{p}<0.001$, n.s: not significant.

(F-G) Confocal detection and quantification of RFP-labeled MADD-4 fluorescence in sdn-1(0) mutants compared to control $(F)$ or in $s d n-1(\triangle 3 G A G)-m N G$ animals compared to control $(G)$. Data were normalized to the mean value of the control group. Mann-Whitney tests ${ }^{* * *} p<0.001,{ }^{*} p<0.05$.

Scale bars $=10 \mu \mathrm{m}$. Violin plot as in figure $1 \mathrm{G}$. See also Fig. S4.

\section{Figure 5. SDN-1 recruits LIN-2/CASK at cholinergic synapses to cluster a7-like N-AChRs.}

(A-B) Confocal detection and quantification of ACR-16-wrmScarlet (A) or UNC-29-RFP (B) fluorescence levels in control and sdn-1 ( $\triangle E Y F A$ ) animals that carry a mutation deleting the SDN-1 C-ter PDZ binding motif.

(C) Confocal detection and quantification of ACR-16-wrmScarlet in control, lin-2(0), and lin-2(0) animals expressing GFP-LIN-2A or GFP-LIN-2B under the control of the muscle-specific Pmyo-3promoter. Oneway ANOVA followed by Tukey's multiple comparison tests. ${ }^{* * *} p<0.001,{ }^{*} p<0.05$, n.s: not significant.

(D) GST pull-down analysis of LIN-2B interaction with the intracellular part of SDN-1, full size (SDN$\left.1_{\mathrm{ICD}}\right)$ or lacking the PDZ binding motif (SDN-1 ${ }_{\mathrm{ICD}} \triangle \mathrm{EFYA}$ ). The samples were analyzed by immunoblotting using an anti-HA antibody. The same membrane was stained with Ponceau $S$ red to show GST expression. Molecular weights (Mw) are shown on the right.

(E) Confocal detection of RFP-LIN-2B and NLG-1-GFP in control and sdn-1(0) animals expressing rfplin-2b and $n l g-1$-gfp under the control of the muscle-specific promoter Pmyo-3. The fluorescence profiles indicate RFP-LIN-2B and NLG-1-GFP fluorescence intensities along the nerve cord from the pictures above.

(F) Pearson's correlation coefficient between RFP-LIN-2B and NLG-1-GFP fluorescence.

(G) Fluorescence levels of RFP-LIN-2B at the dorsal nerve cord (upper panel) or only at GABA synapses (bottom panel) in control or $s d n-1(0)$ animals. Total fluorescence was measured as for other figures. To measure RFP-LIN-2B fluorescence specifically localized at GABA synapses, NLG-1-GFP marker was used to define GABA synaptic regions from processed images and RFP-LIN-2B fluorescence level was quantified in these defined regions (see material and methods for details).

(H) Confocal detection and quantification of mNG-SDN-1 in control and lin-2(0) animals.

(I) Confocal detection of RFP-LIN-2B and mNG-SDN-1 full length (control) or lacking the PDZ binding motif ( $(s d n-1(\triangle E Y F A))$ in animals expressing ifp-lin-2b under the control of the muscle-specific promoter Pmyo-3. The fluorescence profiles indicate RFP-LIN-2B and mNG-SDN-1 fluorescence intensities along the nerve cord from the pictures above.

(J) Pearson's correlation coefficient between RFP-LIN-2B and mNG-SDN-1 full length or lacking the PDZ binding motif EYFA. Individual values are shown as dots. 
(K) Confocal detection and quantification of LIN-2A-GFP in control, $n \lg -1(0)$ and $s d n-1(0) ; n \lg -1(0)$ double mutants. One-way ANOVA followed by Tukey's multiple comparison tests. ${ }^{* * *} p<0.001,{ }^{* *} p<0.01$. Scale bars $=10 \mu \mathrm{m}$. For the quantification of fluorescence levels, data were normalized to the mean value of the control group. Violin plot as in Figure 1G. Mann-Whitney tests for $A, B, F, G, H, J:{ }^{* * *} p<0.001$, ${ }^{* *} p<0.01,{ }^{*} p<0.05$, n.s: not significant. See also Fig. S5.

\section{Figure 6. FRM-3/FARP bridges a7-like N-AChRs with LIN-2/CASK and SDN-1.}

(A) Confocal detection and quantification of ACR-16-wrmScarlet in control, frm-3(0) and frm-3(0) animals expressing full length FRM-3A-GFP, FERM-FA-GFP or FERM-GFP truncations under the control of the muscle-specific Pmyo-3 promoter.

(B) Confocal detection of RFP-labeled GABA ${ }_{A}$ R (RFP-UNC-49) and FRM-3B-GFP in control and sdn$1(0)$ animals expressing frm-3b-gfp under the control of the muscle-specific promoter Pmyo-3. The fluorescence profiles indicate FRM-3B-GFP and RFP-UNC-49 fluorescence intensities along the nerve cord from the pictures above.

(C) Fluorescence level of FRM-3B-GFP in control and $s d n-1(0)$.

(D) Pearson's correlation coefficient between FRM-3B-GFP and GABA bouton.

(E) Confocal detection and quantification of mNG-SDN-1 full length or lacking the KKDEGS motif ( $\triangle K K D E G S)$ in control or frm-3(0) animals.

(F) Confocal detection and quantification of FRM-3B-GFP in control and $s d n-1(\triangle E Y F A)$ animals expressing frm-3b-gfp under the control of the muscle-specific promoter Pmyo-3. Fluorescence levels of FRM-3B-GFP were normalized to the mean of control.

(G) Confocal detection of ACR-16-wrmScarlet in control or in mNG-SDN-1 knock-in animals lacking the KKDEGS motif.

(H) GST pull-down analysis of the binding between HA tagged FERM-FA domain of FRM-3B and the intracellular domain of SDN-1 (SDN-1 $1_{I C D}$ ) by immunoblotting using anti-HA antibody. The stain-free gel was imaged by UV to show GST expression. Predicted molecular weights (Mw) are shown on the right. (I) GST pull-down analysis of ACR-16 TM3-TM4 cytosolic loop binding with HA tagged LIN-2B or FERMFA domain of FRM-3 by immunoblotting using anti-HA antibody. The same membrane was probed by anti-GST antibody to show GST expression. Molecular weights (Mw) are shown on the right.

Scale bars $=10 \mu \mathrm{m}$. For the quantification of fluorescence levels, data were normalized to the mean value of the control group. Violin plot as in Figure 1G. One-way ANOVA followed by Tukey's multiple comparison tests for $A$ and $E:{ }^{* *} p<0.001$, n.s: not significant. Mann-Whitney tests for $C, D$ and $F$ : ${ }^{* * *} \mathrm{p}<0.001,{ }^{*} \mathrm{p}<0.05$, n.s: not significant.

\section{Figure 7. UNC-40 promotes the synaptic localization of ACR-16 and SDN-1.}

(A) Confocal detection and quantification of ACR-16-wrmScarlet fluorescence expressed in control, unc$40(0)$ and unc-40( $\triangle P 3)$ mutants. Kruskal-Wallis test followed by Dunn's post hoc test.

(B) Confocal detection and quantification of mNG-SDN-1 in control and unc-40(0) mutant animals. Mann-Whitney test. 
(C) Confocal detection and quantification of RFP-UNC-40 under the control of muscle-specific Pmyo-3 promoter in control, sdn-1(0), madd-4(0), and madd-4(0); sdn-1(0) animals. One-way ANOVA followed by Tukey's multiple comparison tests.

(D) Confocal detection of RFP-UNC-40 and the presynaptic vesicle marker SNB-1-GFP under the control of the GABAergic neuron-specific promoter Punc-25 in control and sdn-1(0) animals. The fluorescence profiles indicate RFP-UNC-40 and SNB-1-GFP fluorescence intensities along the nerve cord from the pictures above.

(E) Pearson's correlation coefficient between RFP-UNC-40 and GABA boutons. Mann-Whitney test.

Scale bars $=10 \mu \mathrm{m}$. For the quantification of fluorescence levels, data were normalized to the mean value of the control group. Violin plots as in figure $1 \mathrm{G}$. ${ }^{* \star *} \mathrm{p}<0.001,{ }^{*} \mathrm{p}<0.05$, n.s: not significant.

\section{Figure 8. The intracellular domain of SDN-1 recruits N-AChR to GABA synapses.}

(A) Protein structure of the chimeric protein composed of NLG-1 ecto- and transmembrane (TM) domains and SDN-1 intracellular domain (ICD). GFP tag was inserted immediately after the signal peptide (SP). PDZ binding motif of SDN-1 is shown in blue.

(B) Confocal detection of the chimeric protein GFP-NLG-1ecd/SDN-1icd expressed under the control of the muscle-specific promoter Pmyo-3 and the presynaptic marker CLA-1-BFP under the control of the GABAergic neuron-specific promoter Punc-47.

(C) Confocal detection of ACR-16-wrmScarlet and the presynaptic active zone marker CLA-1-BFP under the control of the cholinergic neuron-specific promoter Punc-17 in control and sdn-1(0) animals. Chimeric protein NLG-1ecd/SDN-1icd was expressed under the muscle-specific promotor Pmyo-3 in sdn-1(0) mutant animal. The fluorescence profiles indicate ACR-16-wrmScarlert and CLA-1-BFP fluorescence intensities along the nerve cord from the pictures above after image processing (see materials and methods for details).

(D) Pearson's correlation coefficient between N-AChRs and cholinergic boutons. Mann-Whitney test.

(E) Confocal detection of RFP-labeled L-AChRs (UNC-29-RFP) and the chimeric protein GFP-NLG1ecd/SDN-1icd expressed under the control of the muscle-specific promoter Pmyo-3 in sdn-1(0) mutants. The fluorescence profiles indicate UNC-29-RFP and GFP-Chimera fluorescence intensities along the nerve cord from the pictures above.

(F) Pearson's correlation coefficient between the chimera and N-AChRs or L-AChRs in $s d n-1(0)$ mutants. Mann-Whitney test.

(G) Quantification of UNC-29-RFP in control, $s d n-1(0)$ and $s d n-1(0)$ expressing the chimera specifically in muscle. Fluorescence levels were normalized to the mean of control. Kruskal-Wallis test followed by Dunn's post test.

Scale bars $=10 \mu \mathrm{m}$. Violin plots as in figure $1 \mathrm{G} .{ }^{* * *} p<0.001$, n.s: not significant.

Figure 9. Working model for N-AChR clustering at NMJs.

See discussion for details. 


\section{METHODS}

\section{Strains and genetics}

All C. elegans strains were originally derived from the wild-type Bristol N2 strain. Worm cultivation, genetic crosses, and manipulation of $C$. elegans were carried out according to standard protocols (Brenner, 1974). All strains were maintained on nematode growth medium (NGM) agar plates with Escherichia coli OP50 as a food source at $20^{\circ} \mathrm{C}$. A complete list of strains used in this study can be found in Table 1.

\section{Table 1: List of strains}

\begin{tabular}{|c|c|c|}
\hline Figure & Strain & Genotype \\
\hline $1,5,6,7, S 5$ & EN437 & kr437 [mNG-sdn-1] (X) \\
\hline $1, \mathrm{~S} 1$ & EN7838 & kr296 [unc-49-TagRFP-T] (III) ; kr437 [mNG-sdn-1] (X) \\
\hline $1, \mathrm{~S} 1,5$ & EN7839 & kr208 [unc-29-TagRFP-T] (I); kr437 [mNG-sdn-1] (X) \\
\hline $1, \mathrm{~S} 1$ & EN7860 & krSi144 [Punc-17::cla-1-TagBFP] (I) ; kr437 [mNG-sdn-1] (X) \\
\hline $1, \mathrm{~S} 1$ & EN7915 & krSi143 [Punc-47::cla-1-TagBFP] (V) ; kr437 [mNG-sdn-1] (X) \\
\hline 2 & EN7849 & $\begin{array}{l}\text { kr440 [acr-16-wrmScarlet] (I) ; krSi144 [Punc-17::cla-1-TagBFP] (I) } \\
\text { sdn-1(zh20) (X) }\end{array}$ \\
\hline 2 & EN7850 & kr440 [acr-16-wrmScarlet] (I) ; krSi144 [Punc-17::cla-1-TagBFP] (I) \\
\hline $2, \mathrm{S1}$ & EN208 & kr208 [unc-29-TagRFP-T] (I) \\
\hline 2 & EN2649 & kr208 [unc-29-TagRFP-T] (I) ; sdn-1(zh20) (X) \\
\hline 2 & EN7244 & zxIs6 [Punc-17::ChR2(H134R)-YFP+lin-15(+)] (V) \\
\hline 2 & EN7243 & zxIs6 [Punc-17::ChR2(H134R)-YFP+lin-15(+)] (V) ; sdn-1(zh20) (X) \\
\hline S1 & EN7808 & krSi144 [Punc-17::cla-1-TagBFP] (I) \\
\hline S1 & EN7809 & krSi143 [Punc-47::cla-1-TagBFP] (V) \\
\hline S2 & EN3886 & $\begin{array}{l}\text { kr296 [unc-49-TagRFP-T] (III) ; sdn-1(zh20) }(X) ; \text { krls67 [Punc- } \\
47:: s n b-1-T a g B F P]\end{array}$ \\
\hline S2 & EN3796 & kr296 [unc-49-TagRFP-T] (III) ; krls67 [Punc-47::snb-1-TagBFP] \\
\hline S2 & EN3513 & kr296 [unc-49-TagRFP-T] (III) ; sdn-1(zh20) (X) \\
\hline S1, S2 & EN296 & kr296 [unc-49-TagRFP-T] (III) \\
\hline 3 & EN387 & kr387 [sdn-1::SL2-wrmScarlet] (X) \\
\hline 3 & EN7094 & $\begin{array}{l}\text { kr208 [unc-29-TagRFP-T] (I); krSi50 [Peft-3::TIR1-TagBFP] (IV) ; } \\
\text { kr383 [sdn-1::AID-mNG] (X) }\end{array}$ \\
\hline 3 & EN7158 & $\begin{array}{l}\text { kr208 [unc-29-TagRFP-T] (I) ; krSi55 [Pmyo-3::TIR1-TagBFP] (V); } \\
\text { kr383 [sdn-1::AID-mNG] (X) }\end{array}$ \\
\hline 3 & EN7029 & $\begin{array}{l}\text { kr208 [unc-29-TagRFP-T] (I) ; krSi36 [Prab-3::TIR1-TagBFP] (V); } \\
\text { kr383 [sdn-1::AID-mNG] (X) }\end{array}$ \\
\hline
\end{tabular}




\begin{tabular}{|c|c|c|}
\hline 3 & EN7235 & $\begin{array}{l}\text { kr208 [unc-29-TagRFP-T] (I); krSi63 [Pdpy-7::TIR1-TagBFP] (II); } \\
\text { kr383 [sdn-1::AID-mNG] (X) }\end{array}$ \\
\hline S3 & EN7131 & $\begin{array}{l}\text { ieSi58 [Peft-3::AID-GFP::unc-54 3'UTR + Cbr-unc-119(+)] (IV) ; } \\
\text { krSi50 [Peft-3::TIR1-TagBFP] (IV) }\end{array}$ \\
\hline S3 & EN7157 & $\begin{array}{l}\text { ieSi58 [Peft-3::AID-GFP::unc-54 3'UTR + Cbr-unc-119(+)] (IV); } \\
\text { krSi55 [Pmyo-3::TIR1-TagBFP] (V) }\end{array}$ \\
\hline S3 & EN7028 & $\begin{array}{l}\text { ieSi58 [Peft-3::AID-GFP::unc-54 3'UTR + Cbr-unc-119(+)] (IV) ; } \\
\text { krSi36 [Prab-3::TIR1-TagBFP] (V) }\end{array}$ \\
\hline S3 & EN7236 & $\begin{array}{l}\text { krsi63 [Pdpy-7::TIR1-TagBFP] (II) ; ieSi58 [Peft-3::AID-GFP::unc-54 } \\
\text { 3'UTR + Cbr-unc-119(+)] (IV) }\end{array}$ \\
\hline 4 & EN7937 & krSi145 [Punc-17::cla-1-TagBFP] (IV) ; kr437 [mNG-sdn-1] (X) \\
\hline 4 & EN7938 & $\begin{array}{l}\text { madd-4(kr270) (I) ; krSi145 [Punc-17::cla-1-TagBFP] (IV) ; kr437 } \\
\text { [mNG-sdn-1] (X) }\end{array}$ \\
\hline 4 & EN7747 & $\begin{array}{l}\text { kr373 [madd-4-TagRFP-T] (I); kr437 [mNG-sdn-1] (X);krls67 } \\
\text { [Punc-47::snb-1-TagBFP] }\end{array}$ \\
\hline 4 & EN7754 & $\begin{array}{l}\text { madd-4(tr185) (I) ; kr437 [mNG-sdn-1] (X) ; krSi67 [Punc-47::snb-1- } \\
\text { TagBFP] }\end{array}$ \\
\hline 4 & EN7755 & $\begin{array}{l}\text { madd-4(ttTi103747) (I) ; kr437 [mNG-sdn-1] (X); krSi67 [Punc- } \\
\text { 47::snb-1-TagBFP] }\end{array}$ \\
\hline 4 & EN373 & kr373 [madd-4-TagRFP-T] (I) \\
\hline 4 & EN7899 & kr373 [madd-4-TagRFP-T] (I) ; sdn-1(zh20) (X) \\
\hline 4 & EN7631 & kr373 [madd-4-TagRFP-T] (I) ; kr383 [sdn-1-AID-mNG] (X) \\
\hline 4 & EN7758 & $\begin{array}{l}\text { kr373 [madd-4-TagRFP-T] (I); kr475 [sdn-1(S71A,S86A, S214A)- } \\
\text { AID-mNG] (X) }\end{array}$ \\
\hline S4 & EN383 & kr383 [sdn-1-AID-mNG] (X) \\
\hline S4 & EN441 & $k r 441$ [sdn-1(S71A)-AID-mNG] \\
\hline S4 & EN388 & kr388 [sdn-1(S71A,S86A)-AID-mNG] \\
\hline S4 & EN475 & $k r 475[s d n-1(S 71 A, S 86 A, S 214 A)-A I D-m N G]$ \\
\hline S5 & EN496 & $k r 496[m N G-s d n-1(\Delta E Y F A)](X)$ \\
\hline S5 & EN7858 & krSi143 [Punc-47::cla-1-TagBFP] V; krSi35 [Pmyo-3::lin-2-RFP] \\
\hline S5 & EN7859 & $\begin{array}{l}\text { krSi143 [Punc-47::cla-1-TagBFP] V; krSi35 [Pmyo-3::lin-2-RFP] } \\
\text { kr496 [mNG-sdn-1(AEYFA)] (X) }\end{array}$ \\
\hline $5,6,7$ & EN440 & kr440 [acr-16-wrmScarlet] (I) \\
\hline 5 & EN7556 & kr440 [acr-16-wrmScarlet] (I); kr437 [mNG-sdn-1] (X) \\
\hline 5 & EN7840 & kr440 [acr-16-wrmScarlet] (I) ; kr496 [mNG-sdn-1(AEYFA)] (X) \\
\hline 5 & EN7866 & kr208 [unc-29-TagRFP-T] (I) ; kr496 [mNG-sdn-1(AEYFA)] (X) \\
\hline 5 & EN7729 & kr440 [acr-16-wrmScarlet] (I) ; lin-2 (n397) (X) \\
\hline
\end{tabular}




\begin{tabular}{|c|c|c|}
\hline 5 & EN7903 & $\begin{array}{l}\text { krSi30 [Pmyo-3::lin-2a-GFP] ; kr440 [acr-16-wrmScarlet] (I) ; lin-2 } \\
(n 397)(X)\end{array}$ \\
\hline 5 & EN7960 & $\begin{array}{l}\text { krSi60 [Pmyo-3::lin-2b-GFP] ; kr440 [acr-16-wrmScarlet] (I) ; lin-2 } \\
(n 397)(X)\end{array}$ \\
\hline 5 & EN7057 & krSi35 [Pmyo-3::lin-2a-RFP] ; krSi10 [Pmyo-3::nlg-1-GFP] \\
\hline 5 & EN7779 & $\begin{array}{l}\text { krSi35[Pmyo-3::lin-2a-RFP]; krSi10 [Pmyo-3::nlg-1-GFP] ; sdn- } \\
\text { 1(zh20) }(X)\end{array}$ \\
\hline 5 & EN7720 & kr437 [mNG-sdn-1] $(X) ; \operatorname{lin}-2(n 397)(X)$ \\
\hline 5 & EN7858 & $\begin{array}{l}\text { krSi35 [Pmyo-3::lin-2a-RFP] ; krSi143 [Punc-47::cla-1-TagBFP] ; } \\
\text { kr437 [mNG-sdn-1] (X) }\end{array}$ \\
\hline 5 & EN7859 & 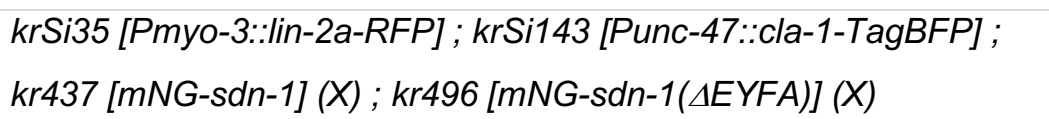 \\
\hline 5 & EN3921 & $\begin{array}{l}\text { krSi30[Pmyo-3::lin-2a-GFP] ; krls67 [Punc-47::snb-1-TagBFP] ; } \\
\text { kr296 [unc-49-TagRFP-T] (III) }\end{array}$ \\
\hline 5 & EN7013 & $\begin{array}{l}\text { krSi30[Pmyo-3::lin-2a-GFP] ; krls67 [Punc-47::snb-1-TagBFP] ; } \\
\text { kr296 [unc-49-TagRFP-T] (III) ; nlg-1 (ok259) (X) }\end{array}$ \\
\hline 5 & EN7832 & $\begin{array}{l}\text { krSi30[Pmyo-3::lin-2a-GFP] ; krls67 [Punc-47::snb-1-TagBFP]; } \\
\text { kr296 [unc-49-TagRFP-T] (III) ; nlg-1 (ok259)] (X) ; sdn-1(zh20) (X) }\end{array}$ \\
\hline 6 & EN7489 & kr440 [acr-16-wrmScarlet] (I) ; frm-3(gk585) (X) \\
\hline 6 & EN7959 & $\begin{array}{l}\text { krSi31 [Pmyo-3::GFP-frm-3a] ; kr440 [acr-16-wrmScarlet] (I) ; frm- } \\
\text { 3(gk585) (X) }\end{array}$ \\
\hline 6 & EN8055 & $\begin{array}{l}\text { krSi32 [Pmyo-3::GFP-frm-3a(1-412aa)] ; kr440 [acr-16-wrmScarlet] } \\
\text { (I) ; frm-3(gk585) (X) }\end{array}$ \\
\hline 6 & EN7912 & $\begin{array}{l}\text { krSi39 [Pmyo-3::GFP-frm-3a(1-318aa)] ; kr440 [acr-16-wrmScarlet] } \\
\text { (I) ; frm-3(gk585) (X) }\end{array}$ \\
\hline 6 & EN8048 & $\begin{array}{l}\text { kr440 [acr-16-wrmScarlet] (I) ; kr437 [mNG-sdn-1] (X) ; frm- } \\
3(g k 585)(X)\end{array}$ \\
\hline 6 & EN50519 & $s d n-1[m N G-k r 519(\Delta K K D E G S)](X)$ \\
\hline 6 & EN8057 & $s d n-1[m N G-k r 519(\Delta K K D E G S)](X) ;$ frm-3(gk585) $(X)$ \\
\hline 6 & EN8082 & kr440 [acr-16-wrmScarlet] (I) ; sdn-1 [mNG-kr519(AKKDEGS)] (X) \\
\hline 6 & EN7177 & krSi61 [Pmyo-3::frm-3-GFP] ; kr296 [unc-49-TagRFP-T] (III) \\
\hline 6 & EN8046 & $\begin{array}{l}\text { krSi144 [Punc-17::cla-1-TagBFP] ; krSi61 [Pmyo-3::frm-3-GFP] ; } \\
\text { kr499 [sdn-1(AEYFA)] (X) }\end{array}$ \\
\hline 6 & EN7208 & $\begin{array}{l}\text { krSi61 [Pmyo-3::frm-3-GFP] ; krls67 [Punc-47::snb-1-TagBFP] ; } \\
\text { kr296 [unc-49-TagRFP-T] (III) }\end{array}$ \\
\hline 6 & EN7209 & $\begin{array}{l}\text { krSi61 [Pmyo-3::frm-3-GFP] ; krls67 [Punc-47::snb-1-TagBFP]; } \\
\text { kr296 [unc-49-TagRFP-T] (III) ; sdn-1(zh20) (X) }\end{array}$ \\
\hline 7 & EN7512 & unc-40(e1430) (I) ; kr440 [acr-16-wrmScarlet] (I) \\
\hline
\end{tabular}




\begin{tabular}{|c|c|c|}
\hline 7 & EN7910 & $\begin{array}{l}\text { krSi145 [Punc-17::cla-1-TagBFP] ; kr457[unc-40(AP3)] (I) ; kr440 } \\
\text { [acr-16-wrmScarlet] (I) }\end{array}$ \\
\hline 7 & EN7733 & unc-40(e1430) (I) ; kr437 [mNG-sdn-1] (X) \\
\hline 7 & EN7833 & $\begin{array}{l}\text { krSi11 [Pmyo-3::TagRFP-T-unc-40] (II) ; juls1 [Punc-25 ::snb-1- } \\
\text { GFP] ; sdn-1(zh20) (X) }\end{array}$ \\
\hline 7 & EN3333 & krSi11 [Pmyo-3::RFP-unc-40] (II) \\
\hline 7 & EN3361 & krSi11 [Pmyo-3::RFP-unc-40] (II) ; juls1 [Punc-25 ::snb-1-GFP] \\
\hline 7 & EN7845 & krSi11 [Pmyo-3::TagRFP-T-unc-40] (II) ; madd-4(kr270) (I) \\
\hline 7 & EN7831 & $\begin{array}{l}\text { krSi11 [Pmyo-3::Tag-RFP-T-unc-40] (II) ; madd-4(kr270) (I) ; sdn- } \\
\text { 1(zh20) (X) }\end{array}$ \\
\hline 8 & EN8107 & $\begin{array}{l}\text { krSi157[Pmyo-3::GFP-NLG-1ecd/SDN-1/icd] ; krSi143 [Punc- } \\
\text { 47::cla-1-TagBFP] ; kr296 [unc-49-TagRFP-T] (III) }\end{array}$ \\
\hline 8 & EN7926 & $\begin{array}{l}\text { krSi157[Pmyo-3::GFP-NLG-1ecd/SDN-1/icd] ; krSi145 [Punc- } \\
\text { 17::cla-1-TagBFP] ; kr440 [acr-16-wrmScarlet] (I) ; sdn-1(zh20) (X) }\end{array}$ \\
\hline 8 & EN7851 & $\begin{array}{l}\text { krSi144 [Punc-17::cla-1-TagBFP]; kr208 [unc-29-TagRFP-T] (I); } \\
\text { sdn-1(zh20) (X) }\end{array}$ \\
\hline 8 & EN8040 & $\begin{array}{l}\text { krSi160[Pmyo-3::GFP-NLG-1ecd/SDN-1/icd] ; kr208 [unc-29- } \\
\text { TagRFP-T] (I) ; sdn-1(zh20) (X) }\end{array}$ \\
\hline
\end{tabular}

\section{Plasmids}

The constructs created in this study are described in Table 2. The full open reading frames of all constructs were verified by Sanger sequencing from GATC Company. The other plasmids used to create single-copy insertion alleles by miniMos method are described in a previous study (Zhou et al., 2020).

Table 2: List of generated plasmids

\begin{tabular}{|l|l|l|}
\hline Plasmid & Description & Usage \\
\hline pUA111 & TIR-1-TagBFP & $\begin{array}{l}\text { MiniMos plasmid to build tissue-specific } \\
\text { expression of Transport Inhibitor } \\
\text { Response 1 (TIR1) alleles }\end{array}$ \\
\hline pDD268 & mNG-3xFLAG & $\begin{array}{l}\text { Template used to insert homology arms to } \\
\text { create } s d n-1: \text { AID-mNG (EN383) strain }\end{array}$ \\
\hline pAV52 & TagRFP-T & $\begin{array}{l}\text { Repair template to create EN373 CRISPR } \\
\text { knock-in strain }\end{array}$ \\
\hline pCV01 & AID-mNG & $\begin{array}{l}\text { Repair template to create EN383 CRISPR } \\
\text { knock-in strain }\end{array}$ \\
\hline
\end{tabular}




\begin{tabular}{|c|c|c|}
\hline pCV02 & SL2:::wrmScarlet & $\begin{array}{l}\text { Repair template to create EN387 CRISPR } \\
\text { knock-in strain }\end{array}$ \\
\hline pCV03 & PU6pol(III)::crCV02 & $\begin{array}{l}\text { Vector expressing guide RNA to create } \\
\text { EN387 strain }\end{array}$ \\
\hline pCV04 & $\begin{array}{l}\text { Pmyo-3::TIR1-TagBFP::unc-54 } \\
\text { 3'UTR }\end{array}$ & $\begin{array}{l}\text { Minimal Mos } 1 \text { vector for Pmyo-3::TIR1- } \\
\text { TagBFP insertion. This plasmid was used } \\
\text { to create } k r S i 55 \text { allele. Contains a } \\
\text { neomycin-resistant cassette }\end{array}$ \\
\hline pCV05 & Prab-3::TIR1-TagBFP::unc-54 3'UTR & $\begin{array}{l}\text { Minimal Mos } 1 \text { vector for Prab-3::TIR1- } \\
\text { TagBFP insertion. This plasmid was used } \\
\text { to create } k r S i 36 \text { allele. Contains a } \\
\text { neomycin-resistant cassette }\end{array}$ \\
\hline pCV08 & Pdpy-7::TIR1-TagBFP::unc-54 3'UTR & $\begin{array}{l}\text { Minimal Mos } 1 \text { vector for } P d p y-7:: T I R 1- \\
\text { TagBFP insertion. This plasmid was used } \\
\text { to create } k r S i 63 \text { allele. Contains a } \\
\text { neomycin-resistant cassette }\end{array}$ \\
\hline pCV09 & Peft-3::TIR1-TagBFP::unc-54 3'UTR & $\begin{array}{l}\text { Minimal Mos } 1 \text { vector for Peft-3::TIR1- } \\
\text { TagBFP insertion. This plasmid was used } \\
\text { to create } k r S i 50 \text { allele. Contains a } \\
\text { neomycin-resistant cassette }\end{array}$ \\
\hline $\mathrm{pXZ48}$ & $p c D N A 3.1(+)-H A-l i n-2 b$ & $\begin{array}{l}\text { To synthesize HA-LIN-2B in vitro by } \mathrm{TnT} \AA \\
\text { kit. }\end{array}$ \\
\hline pXZ64 & pcDNA3.1(+)-frm-3_FERM-FA & $\begin{array}{l}\text { To synthesize HA-FERM-FA domain of } \\
\text { FRM-3 in vitro by TnT } ® \text { kit. }\end{array}$ \\
\hline pXZ92 & pGEX-3X-SDN-1_ICD & $\begin{array}{l}\text { To express GST-SDN-1 intracellular } \\
\text { domain in arctic express E. coli }\end{array}$ \\
\hline pXZ94 & pGEX-3X-SDN-1_ICDAEYFA & $\begin{array}{l}\text { To express GST-SDN-1 intracellular } \\
\text { domain lacking EYFA PDZ binding motif in } \\
\text { arctic express E.coli }\end{array}$ \\
\hline pXZ97 & pGEX-3X-ACR-16_loop & $\begin{array}{l}\text { To express GST-ACR-16 TM3-4 loop in } \\
\text { arctic express E. coli }\end{array}$ \\
\hline pXZ102 & $\begin{array}{l}\text { Pmyo-3::sp-mNG-nlg-1Ecd+TM/sdn- } \\
\text { 1/cd }\end{array}$ & $\begin{array}{l}\text { Minimal Mos } 1 \text { vector to express NLG-1 } \\
\text { ecto-transmembrane domain and SDN-1 } \\
\text { intracellular domain in body-wall muscle }\end{array}$ \\
\hline
\end{tabular}

\section{Generation of single-copy insertion alleles}

The single-copy insertion alleles generated by miniMos method (Frøkjær-Jensen et al., 2014) are listed in Table 3. Genes encoding fluorescent tagged proteins are driven by myo-3 (body-wall muscle), unc-47 (GABAergic motoneuron), unc-17 (cholinergic motoneuron), rab-3 (pan-neuronal), dpy-7 
(epidermis), or eft-3 (universal) specific promoters. N2 animals were injected with $15 \mathrm{ng} / \mu \mathrm{L}$ of plasmid of interest containing the promoter and the open reading frame, $50 \mathrm{ng} / \mu \mathrm{L} \mathrm{pCFJ601} \mathrm{(Mos1} \mathrm{transposase),}$ $10 \mathrm{ng} / \mu \mathrm{L}$ pMA122 (negative selective marker Phsp16.2::peel-1), $2.5 \mathrm{ng} / \mu \mathrm{L}$ pCFJ90 (Pmyo-2::mCherry). Neomycin (G418) was added to plates 24 hours after injection at $1.5 \mu \mathrm{g} / \mu \mathrm{L}$ final concentration. Candidate plates were heat-shocked for 2 hours at $34^{\circ} \mathrm{C}$ to remove extrachromosomal arrays and then homozygosed.

\section{Table 3: List of miniMos single-copy insertion alleles}

\begin{tabular}{|l|l|}
\hline Alleles name & Construct \\
\hline krSi143 & Punc-47::cla-1-TagBFP V \\
\hline krSi144 & Punc-17::cla-1-TagBFP I \\
\hline krSi145 & Punc-17::cla-1-TagBFP IV \\
\hline krSi50 & Peft-3::TIR1-TagBFP IV \\
\hline krSi55 & Pmyo-3::TIR1-TagBFP V \\
\hline krSi36 & Prab-3::TIR1-TagBFP V \\
\hline krSi63 & Pdpy-7::TIR1-TagBFP II \\
\hline krSi157 & Pmyo-3::mNG-NLG-1ecd/SDN-1icd, line1 \\
\hline krSi160 & Pmyo-3::mNG-NLG-1ecd/SDN-1icd, line2 \\
\hline krSi30 & Pmyo-3::sfGFP-lin-2a \\
\hline krSi31 & Pmyo-3::frm-3-sfGFP \\
\hline krSi32 & Pmyo-3::frm-3(1-412aa)-sfGFP \\
\hline krSi35 & Pmyo-3::tagRFP-T-lin-2a \\
\hline krSi39 & Pmyo-3::frm-3(1-318aa)-sfGFP \\
\hline krSi60 & Pmyo-3::sfGFP-lin-2b \\
\hline krSi61 & Pmyo-3:::sfGFP-frm-3b \\
\hline
\end{tabular}

\section{Modifications of the endogenous loci}

To generate the kr383 allele, the AID sequence (Zhang et al., 2015) and mNG were inserted tandemly into the sdn-1 locus, just before the stop codon. To generate the $k r 373$ and $k r 387$ alleles, the TagRFP-T and the wrmScarlet (Bindels et al., 2017; El Mouridi et al., 2017) sequences were inserted in the madd-4 and in the $s d n-1$ loci, respectively, just before the stop codon. The detailed protocol was described in (Dickinson et al., 2015). CrRNA sequences targeting the insertion region were designed and synthesized from IDT (Integrated DNA Technologies Inc.). CrRNA and tracerRNA (trRNA) were mixed as 1:1 ratio to form a sgRNA duplex. Adult worms were micro-injected in the gonads with a mix that contained: Cas9 nuclease $0.5 \mu \mathrm{L}$ of $10 \mu \mathrm{g} / \mu \mathrm{L}$ (IDT Inc.), sgRNA duplex $3 \mu \mathrm{L}(100 \mu \mathrm{M})$, repair templates (containing the DNA fragment to insert, a hygromycin resistance selection cassette surrounded by loxP sites, Phsp16.41::Cre and homology arms to target the genomic region) $50 \mathrm{ng} / \mathrm{\mu L}$, pCFJ90 [Pmyo-2::mCherry] co-injection marker $2.5 \mathrm{ng} / \mu \mathrm{L}$, RNase/DNase free water up to $10 \mu \mathrm{L}$. Injected animals were then grown at $25^{\circ} \mathrm{C}$, and after $48 \mathrm{~h}$, a positive selection was performed by adding 
hygromycin on plates at $0.2 \mu \mathrm{g} / \mu \mathrm{L}$. Resistant animals were then heat-shocked for $2 \mathrm{~h}$ at $34^{\circ} \mathrm{C}$ by water bath to excise the hygromycin selection cassette from the genome. Worms were then screened by sequencing PCR products from the region of interest. Candidates were outcrossed once with N2 to remove unspecific background mutations.

To generate the $k r 437$ and $k r 440$ alleles, PCR amplified sequences of mNG or wrmScarlet with or without homology arms were used as repair templates respectively (Dokshin et al., 2018). CrRNA was designed and synthesized by IDT (Integrated DNA Technologies Inc.). The injection mix contained annealed dsDNA donor cocktail as repair template $200 \mathrm{ng} / \mu \mathrm{L}$ (total $4 \mu \mathrm{g}$ ), Cas9 nuclease $0.5 \mu \mathrm{L}$ of 10 $\mu \mathrm{g} / \mu \mathrm{L}$ (IDT Inc.), sgRNA duplex $3 \mu \mathrm{L}(100 \mu \mathrm{M})$. The candidate F1 animals were isolated by tracking the initial fluorescence knock-in. The F2 progenies were then isolated and homozygosed. The insertion was then confirmed by PCR and sequencing.

To generate $k r 388, k r 441, k r 475, k r 496, k r 499$ and $k r 519$, ultramer DNA repair templates were produced by IDT Inc.. The injection mix contained: Cas9 nuclease $0.5 \mu \mathrm{L}$ of $10 \mu \mathrm{g} / \mu \mathrm{L}$ (IDT Inc.), sgRNA duplex $3 \mu \mathrm{L}(100 \mu \mathrm{M})$, ultramer repair template $1.25 \mu \mathrm{L}(100 \mu \mathrm{M})$, pRF4 [Peft-3::rol-6 ] co-injection marker $2.5 \mathrm{ng} / \mu \mathrm{L}$, RNase/DNase free water filled up to $10 \mu \mathrm{L}$. Insertions in F1 roller progenies were checked by PCR. Homozygotes were isolated and the PCR product were then sent for sequencing. Candidates were outcrossed once with N2 to remove unspecific background mutations.

An extensive list of modified genomic loci can be found in Table 4.

\section{Table 4: List of modified genomic loci}

\begin{tabular}{|c|c|}
\hline Alleles name & Modification \\
\hline kr208 & TagRFP-T inserted into TM3-4 loop of UNC-29 \\
\hline$k r 270$ & Full deletion of madd-4 locus \\
\hline$k r 296$ & TagRFP-T inserted at N-terminus of UNC-49 \\
\hline$k r 373$ & TagRFP inserted at C-terminus of MADD-4L/B \\
\hline kr383 & AID-mNG fusion tag inserted right after PDZ binding motif of SDN-1 \\
\hline kr387 & SL2:: wrmScarlet tag inserted right after PDZ binding motif of SDN-1 \\
\hline$k r 441$ & S71A in sdn-1-aid-mNG (sdn-1(D1GAG)) \\
\hline$k r 388$ & S71A and S86A in sdn-1-aid-mNG (sdn-1(D2GAG)) \\
\hline kr475 & S71A, S86A and S214A in sdn-1-aid-mNG (sdn-1(D3GAG)) \\
\hline kr437 & $m N G$ inserted at N-terminus (after signal peptide) of SDN-1 \\
\hline kr440 & wrmScarlet inserted into TM3-4 loop of ACR-16 \\
\hline kr457 & Deletion of P3 motif in UNC-40 \\
\hline$k r 496$ & Deletion of PDZ binding motif (EYFA) in mNG-SDN-1 \\
\hline$k r 499$ & Deletion of PDZ binding motif (EYFA) in N2 wild type \\
\hline kr519 & Deletion of Ezrin binding motif (KKDEGS) in mNG-SDN-1 \\
\hline
\end{tabular}

\section{Auxin-induced degradation}


Auxin plates were prepared by adding auxin Indole-3-Acetic Acid (Sigma) from a $400 \mathrm{mM}$ stock solution in ethanol into NGM at the final concentration of $1 \mathrm{mM}$ (Zhang et al., 2015). Tissue-specific degradation efficiency was monitored on animal expressing GFP fused to the Auxin-Inducible Domain (AID) under the ubiquitous promoter Peft-3 and Prab-3::TIR1-TagBFP, Peft-3::TIR1-TagBFP, Pdpy7::TIR1-TagBFP or Pmyo-3::TIR1-TagBFP. Animals were grown on regular or auxin plates for 3, 36, 96 and 120 hours respectively. SDN-1-AID-mNG fluorescence was scored after tissue-specific degradation on young adult animals that had hatched on regular or auxin plates.

\section{Microscopy imaging and quantification}

For confocal imaging, young live adult hermaphrodites (24h post L4 larval stage) were mounted on $2 \%$ agarose dry pads with $5 \%$ poly-lysine beads in $\mathrm{M} 9$ buffer $\left(3 \mathrm{~g}\right.$ of $\mathrm{KH}_{2} \mathrm{PO}_{4}, 6 \mathrm{~g}$ of $\mathrm{Na}_{2} \mathrm{HPO}_{4}, 5 \mathrm{~g}$ of

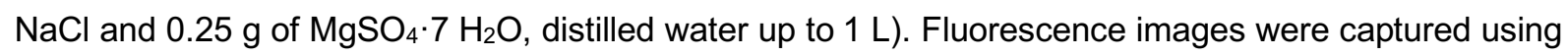
an Andor spinning disk system (Oxford Instruments) installed on a Nikon-IX86 microscope (Olympus) equipped with a 60x/NA1.2 oil immersion objective and an Evolve EMCCD camera. Each animal was imaged as a stack of optical sections $(0.2 \mu \mathrm{m}$ apart) and 41 plans were projected along Z-axis.

Quantification of images was performed using Image ( 1.48 by NIH) with Fiji plugin add-ons. For fluorescence intensity measurement, $50 \mu \mathrm{m}$ (wide) $\times 3 \mu \mathrm{m}$ (high) regions along the dorsal cord near the mid-body were cropped and analyzed. Acquisition settings were the same across genotypes for quantitative analysis. Each strain was imaged on at least 3 different days, and the data were pooled together. Data are presented as a percentage of the average fluorescence relative to that of the control strain. For colocalization quantification, images were captured along $40 \mu \mathrm{m}$ of the dorsal nerve cord anterior to the vulva. The fluorescence intensity along the cord was evaluated with the Plot Profile plugin. For each channel, the values along the $x$-axis were normalized to the value of maximal intensity. Data are presented as min to max values for animals of each genotype. The colocalization between two channels were analyzed by Pearson's correlation coefficient as described previously (Pinan-Lucarré et al., 2014).

To quantify the intensity of mNG-SDN-1 at ACh vs GABA NMJs (Fig. 1H), images of the dorsal cord of animals co-expressing $m N G$-sdn-1 and Punc-17::cla-1-BFP or Punc-47::cla-1-BFP were acquired and cropped for quantification. In both channels, the background was subtracted using a rolling ball radius of 10 pixels in Fiji (Image $\mathrm{J}$ ). The CLA-1-BFP channel of cropped images was converted to binary using the Ostu threshold. For Punc-17::CLA-1-BFP, noise was removed from the binary mask using the de-speckle function (twice). The thresholded image was then multiplied with the binary mask to generate the preprocessed image for further analysis. To generate the plot profiles, the intensities of each pixel along the $\mathrm{X}$ axis (nerve cord was converted to two-dimensional axis) were recorded for each channel in .csv files. To determine the level of mNG-SDN-1 at cholinergic synapses, the csv files were then read by an $\mathrm{R}$ script to define the cholinergic CLA-1-BFP domains based on values of Punc-17::CLA-1-BFP signals detected along the nerve cord. Next, the mean intensity of SDN-1 was calculated by measuring the area under the curve of SDN-1 profiles within cholinergic CLA-1-BFP domains and then dividing by the cholinergic CLA-1-BFP domain area. The same procedure was applied to determine the level of mNG-SDN-1 at GABAergic synapses and was also used in figure S4D to determine the level of RFP- 
LIN-2 level at GABA synapses. To quantify the percentage of L-ACh and GABA receptors colocalized with SDN-1 (Fig. 1G), the mNG-SDN-1 domains were defined by the same processing as described above for CLA-1-BFP. Next, the percentage of colocalized receptor was calculated by determining the ratio between the area under the curve (in receptor channel) within SDN-1 domains and the area under the curve (in receptor channel) out of the SDN-1 domains. The plot profile fluorescence signals were measured and processed by Mander's overlapping correlation analysis modified from previous studies (Bolte and Cordelières; Dunn et al., 2011).

To quantify RFP-LIN-2 levels at GABA synapses in figures 6G, GABA domains were defined by the same processing as described for CLA-1-BFP above, using NLG-1-GFP as GABAergic synapse marker. To analyze colocalization between N-AChR and cholinergic boutons in figure $9 \mathrm{C}-\mathrm{D}$, images were processed as described in the previous paragraph.

\section{GST-pull down assay and heparinase treatment}

GST-pull down experiment was performed based on previous protocol (Zhou et al., 2016). DNA fragments encoding the ACR-16 TM3-4 loop or the SDN-1 intracellular domain full length or lacking the EYFA PDZ binding motif were cloned to pGEX-3X expression vector and then transfected into Arctic express E.coli strain (Agilent). Bacterial clones were first cultured overnight in $5 \mathrm{~mL}$ LB medium supplemented with $100 \mathrm{mg} / \mathrm{mL}$ ampicillin. The volume of overnight-grown bacteria was enlarged in 100 $\mathrm{mL}$ LB/ampicillin medium by culturing 2 hours at $37^{\circ} \mathrm{C}$ with shaking until OD600 reached 0.6-0.8. IPTG was added to medium at $1 \mathrm{mM}$ final concentration to induce protein expression at $12^{\circ} \mathrm{C}$ for 24 hours. The induced bacteria were collected by centrifugation and lysed by $20 \mathrm{~mW}$ pulsed sonication for $30 \mathrm{~min}$ in cold bacteria lysis buffer (50 mM HEPES pH 7.5, $400 \mathrm{mM} \mathrm{NaCl}, 1 \mathrm{mM}$ DTT, $1 \mathrm{mM}$ PMSF, 1 tablet of protease inhibitor cocktail/50 $\mathrm{mL}$ (Roche-Merck)). Protein lysate was centrifuged at $15,000 \mathrm{xg}$ for 45 min at $4^{\circ} \mathrm{C}$. Clear supernatants were incubated with $100 \mu \mathrm{L}$ Glutathione sepharose-4B beads (Sigma) at $4^{\circ} \mathrm{C}$ for $12 \mathrm{~h}$. To express HA (Human influenza hemagglutinin) tagged LIN-2B, FRM-3B or FERM-FA domain, coding sequences with HA N-terminal tagged were cloned into pcDNA3.1(+) expression vector. Fusion proteins were expressed by TnT® Quick Coupled system (Promega). $15 \mu \mathrm{L}$ of HA tagged protein was then incubated with candidate GST-coated beads at $4^{\circ} \mathrm{C}$ for 2 hours. The beads were washed five times with STE (10 mM Tris-Cl pH8.0, $150 \mathrm{mM} \mathrm{NaCl}, 1 \mathrm{mM}$ EDTA, 0.1\% Tween-20) buffer. Proteins were eluted by adding equal volume of 2xSDS sampling buffer (Bio-rad) and boiled for $10 \mathrm{~min}$. Samples were electrophoresed in 12\% SDS/PAGE (Bio-rad). The separated proteins were incubated with an antiHA antibody (\#3724, Cell signaling Tech.) at 1:1000 dilution. The GST fusion loading amount was detected by stain free gel imaging or Ponceau $S$ red staining immediately after membrane transfer.

To digest heparan sulfate chains of SDN-1 and to compare with GAG binding site mutant animals, mNG-SDN-1 non-mutated or $\Delta 1,2$ and 3 GAGs fusion proteins were first concentrated by immunoprecipitation of roughly 20,000 nematodes per genotype using Trap-A bead coupled with antimNG nanobody (Chromotek). $1.5 \mathrm{U}$ of heparanase I and III (Sigma) was added to non-mutated tube containing $500 \mu \mathrm{L}$ washing buffer (50 mM HEPES, pH 7.7, $50 \mathrm{mM} \mathrm{NaCl}$ ) and 100 Trap-A beads. The tube was then incubated at $37^{\circ} \mathrm{C}$ for 2 hours. The proteins of each group were eluted by adding $100 \mu \mathrm{L}$ 
2x sampling buffer and boiled for $10 \mathrm{~min}$. Samples were separated by $12 \%$ SDS/PAGE (Bio-rad) and blotted with anti-mNG antibody at 1:1000 dilution. N2 wild-type animals were used as negative control.

\section{Electrophysiology}

Adult nematodes were glued (Histoacryl Blue, B. Braun) along the dorsal side of the body to the surface of a plastic culture dish (Corning). A sharpened tungsten rod (A-M systems Inc) was used to perform a lateral incision before the vulva and to remove the viscera. The cuticle flap was glued back to expose the ventral body-wall muscles and the preparation was treated by collagenase type IV for $20 \mathrm{~s}$ at the concentration of $0.5 \mathrm{mg} / \mathrm{mL}$. Recordings were performed on the two first ventromedial muscle cells before the vulva from the right muscle quadrant. For all recordings, strains express ChR2 in cholinergic motoneurons (zx/s6) (Liewald et al., 2008). Animals were grown on NGM plates freshly seeded with $300 \mu \mathrm{L}$ of OP50 culture supplemented by $0.25 \mu \mathrm{L}$ of $100 \mathrm{mM}$ all-trans retinal stock (dissolved in ethanol). Membrane currents were recorded in the whole-cell configuration using a MultiClamp 700B amplifier (Molecular Devices). Acquisition and command voltage were done using the Clampex 10 software driving an Axon Digidata 1550 (Molecular Devices). The resistance of recording pipettes ranged between 3 and $4 \mathrm{M} \Omega$. Data were analyzed with Clampfit 10 (Molecular Devices) and graphed with Origin software (OriginLab). The bath solution contained (in $\mathrm{mM}$ ) $150 \mathrm{NaCl}, 5 \mathrm{KCl}, 5 \mathrm{CaCl}_{2}$,

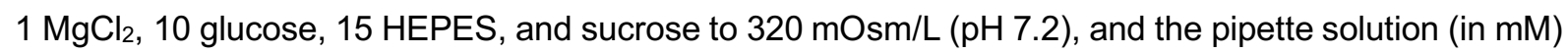
$120 \mathrm{KCl}, 4 \mathrm{NaCl}, 5 \mathrm{EGTA}, 10 \mathrm{TES}, 4 \mathrm{MgATP}$ and sucrose to $310 \mathrm{mOsm} / \mathrm{L}(\mathrm{pH} 7.2)$.

For currents evoked by cholinergic motoneuron stimulation, the slit worm preparations were exposed to $10 \mathrm{~ms}$ light stimulation performed with the pE2 system (CoolLED) at a wavelength of 460 $\mathrm{nm}$ drived by Clampex 10 software. The protocol was the following: a first stimulation triggered the activation of L-AChR and N-AChR; the peak current value was measured and named total peak current. The preparation was then perfused with bath solution supplemented with dihydro-beta-erythroidine $(\mathrm{DH} \beta \mathrm{E})$ at the concentration of $0.01 \mathrm{mM}$ leading to the specific inhibition of N-AChR. In these conditions, a second stimulation was performed activating only L-AChR. A third stimulation was then applied to check if the current was stable and the value of the peak current was measured and named L-AChR evoked current. Finally, for each cell, the L-AChR evoked current was subtracted from the total peak current to obtain the N-AChR evoked current values.

For agonist-evoked currents, nicotine and levamisole diluted at the concentration of $0.1 \mathrm{mM}$ in the bath solution were pressured-ejected during $100 \mathrm{~ms}$ from a pipette similar in size to the patch pipette at 10 psi using a PDES-2DX system (NPI electronic). The slit worm preparations were continuously perfused with fresh bath solution via a gravity flow delivery system. All chemicals were obtained from Sigma-Aldrich except $\mathrm{DH} \beta \mathrm{E}$ provided by Tocris. All experiments were done at $20^{\circ} \mathrm{C}$. 


\section{SUPPLEMENTAL INFORMATION}

Figure S1. mNG insertion in the sdn-1 locus has no effect on NMJ organization.

(A) Confocal detection of the presynaptic active zone marker CLA-1-BFP under the control of the cholinergic neuron-specific promoter Punc-17 in control and $m N G$-sdn-1 knock-in animals. Bottom: quantification of the number of active zones per $50 \mu \mathrm{m}$ of dorsal cord in control and $m N G-s d n-1$ animals.

(B) Confocal detection and quantification of RFP-labeled L-AChR in control and $m N G$-sdn-1 animals. Fluorescence levels were normalized to the mean value of control. Mann-Whitney test, n.s: not significant.

(C) Confocal detection of the presynaptic active zone marker CLA-1-BFP under the control of the GABAergic neuron-specific promoter Punc-47 in control and $m N G$-sdn-1 animals. Bottom: quantification of the number of active zones per $50 \mu \mathrm{m}$ of dorsal cord in control and mNG-sdn-1 worms.

(D) Confocal detection and quantification of RFP-labeled $\mathrm{GABA}_{\mathrm{A}} \mathrm{R}$ in control and $m N G$-sdn-1 animals. Fluorescence levels were normalized to the mean value of control.

Scale bars $=10 \mu \mathrm{m}$. Violin plots as in figure $1 \mathrm{G}$. Mann-Whitney tests, n.s: not significant.

\section{Figure S2. SDN-1 has a moderate effect on $\mathrm{GABA}_{A} R$ synaptic content.}

(A) Confocal detection of the presynaptic active zone marker CLA-1-BFP under the control of the GABAergic neuron-specific promoter Punc-47 in control and sdn-1(0) mutant animals. Bottom: quantification of the number of active zones per $50 \mu \mathrm{m}$ of dorsal cord in control and $s d n-1(0)$ worms.

(B) Confocal detection and quantification of RFP-labeled $\mathrm{GABA}_{A} \mathrm{R}$ in control and $s d n-1(0)$ animals. Fluorescence levels were normalized to the mean value of control.

Scale bars $=10 \mu \mathrm{m}$. Violin plots as in figure 1G. Mann-Whitney tests, ${ }^{* * *} p<0.0001$, n.s: not significant.

Figure S3. The Auxin-Inducible-Degron (AID) leads to tissue-specific degradation.

Confocal detection of AID-GFP expressed in all tissues by the ubiquitous promoter Peft-3 from animals grown on regular NGM plates (control, left panels) or on auxin plates (right panels). Tissue-specific degradation was achieved by co-expressing AID-GFP and Peft-3::tir-1-bfp (ubiquitous), Pdpy-7::tir-1bfp (epidermis), Pmyo-3::tir-1-bfp (body-wall muscle), or Prab-3::tir-1-bfp (neurons). White arrowheads indicate the position of the nerve ring.

Scale bars $=10 \mu \mathrm{m}$.

Figure S4. Heparan sulfate chains modulate SDN-1 synaptic content but are dispensable for its MADD-4-dependent synaptic localization.

(A) Protein structure of SDN-1-mNG with the position of GAG chain anchoring serines. Ser71, Ser86 or Ser214 were mutated into Alanine in mutants lacking one ( $\triangle 1 G A G)$, two $(\triangle 2 G A G)$ or three ( $\triangle 3 G A G)$ GAG chain anchoring sites. Detailed domain information as in Fig $1 \mathrm{~A}$.

(B) Western blot showing the migration of non-mutated SDN-1-mNG (lane 2), SDN-1( $\triangle 3 G A G$ )-mNG (lane 3), SDN-1( $\triangle 2 \mathrm{GAG}$ )-mNG (lane 4), SDN-1( $1 \mathrm{GAG}$ )-mNG (lane 5) protein precipitated from worm 
lysate. Expected SDN-1-mNG molecular weight is $58 \mathrm{kDa}$. Bracket likely indicates the SDS resistant dimerization form of SDN-1. Black arrowhead indicates potential cleaved C-terminus-mNG band. Protein samples from lanes 1-5 were undigested while sample from lane 6 (shown in red) was digested by Heparinase I and III for 2 hours at $37^{\circ} \mathrm{C}$. Protein lysate from N2 wild-type animals was used as control in lane 1.

(C) Confocal detection of SDN-1-mNG with no modification or CRISPR mutation of one ( $\triangle 1 \mathrm{GAG}$ ), two $(\triangle 2 \mathrm{GAG})$ or three $(\triangle 3 \mathrm{GAG}) \mathrm{GAG}$ chain anchoring sites as specified in A. Fluorescence levels were normalized to the mean of non-mutated SDN-1-mNG fluorescence. One-way ANOVA followed by Tukey's multiple comparison tests. ${ }^{* * *} p<0.001$, n.s: not significant. Violin plot as in figure $1 \mathrm{G}$.

Scale bars $=10 \mu \mathrm{m}$.

Figure S5. LIN-2 is concentrated at GABA synapses in animals lacking SDN-1 PDZ binding motif. (A) Confocal detection and quantification of mNG-SDN-1 fluorescence levels in control ( $m N G$-sdn-1) and $m N G-s d n-1(\triangle E Y F A)$ animals. Fluorescence levels were normalized to the mean value of control animals.

(B) Confocal detection of RFP-LIN-2B and the presynaptic active zone marker CLA-1-BFP at GABA synapses in control and sdn-1(AEYFA) animals expressing Pmyo-3::rfp-lin-2b and Punc-47::cla-1-bfp. The fluorescence profiles indicate muscle-expressed RFP-LIN-2B and GABA synaptic CLA-1-BFP fluorescence intensities along the nerve cord from the pictures above.

(C) Pearson's correlation coefficient between RFP-LIN-2B and the GABAergic presynaptic active zone marker CLA-1-BFP in control and sdn-1( $\triangle E Y F A$ ) animals.

(D) Fluorescence levels of RFP-LIN-2B at the dorsal nerve cord or only at GABA synapses of control and $s d n-1(\triangle E Y F A)$ animals. Total fluorescence (left panel) was measured as for other figures. To measure RFP-LIN-2B fluorescence specifically localized at GABA synapses (right panel), GABA synaptic CLA-1-BFP marker was used to define GABA regions from processed images and RFP-LIN$2 \mathrm{~B}$ fluorescence was quantified in these defined regions (see material and methods for details). Fluorescence levels were quantified and normalized to the control groups.

Scale bars $=10 \mu \mathrm{m}$. Violin plots as in figure $1 \mathrm{G}$. Mann-Whitney tests, ${ }^{* * *} p<0.001,{ }^{*} p<0.05$, n.s: not significant. 


\section{REFERENCES}

Alexander, M., Chan, K.K.M., Byrne, A.B., Selman, G., Lee, T., Ono, J., Wong, E., Puckrin, R., Dixon, S.J., and Roy, P.J. (2009). An UNC-40 pathway directs postsynaptic membrane extension in Caenorhabditis elegans. Development 136, 911-922.

Alkondon, M., Pereira, E.F., Barbosa, C.T., and Albuquerque, E.X. (1997). Neuronal nicotinic acetylcholine receptor activation modulates gamma-aminobutyric acid release from CA1 neurons of rat hippocampal slices. J. Pharmacol. Exp. Ther. 283, 1396-1411.

Allen, N.J., Bennett, M.L., Foo, L.C., Wang, G.X., Chakraborty, C., Smith, S.J., and Barres, B.A. (2012). Astrocyte glypicans 4 and 6 promote formation of excitatory synapses via GluA1 AMPA receptors. Nature 486, 410-414.

Apte, S.S. (2009). A Disintegrin-like and Metalloprotease (Reprolysin-type) with Thrombospondin Type 1 Motif (ADAMTS) Superfamily: Functions and Mechanisms. J Biol Chem 284, 31493-31497.

Araque, A., Parpura, V., Sanzgiri, R.P., and Haydon, P.G. (1999). Tripartite synapses: glia, the unacknowledged partner. Trends Neurosci. 22, 208-215.

Asundi, V.K., and Carey, D.J. (1995). Self-association of N-Syndecan (Syndecan-3) Core Protein Is Mediated by a Novel Structural Motif in the Transmembrane Domain and Ectodomain Flanking Region. J. Biol. Chem. 270, 26404-26410.

Attreed, M., Desbois, M., van Kuppevelt, T.H., and Bülow, H.E. (2012). Direct visualization of specifically modified extracellular glycans in living animals. Nat. Methods 9, 477-479.

Babu, K., Hu, Z., Chien, S.-C., Garriga, G., and Kaplan, J.M. (2011). The Immunoglobulin super family protein RIG-3 prevents synaptic potentiation and regulates Wnt signaling. Neuron 71, 103-116.

Ballivet, M., Alliod, C., Bertrand, S., and Bertrand, D. (1996). Nicotinic acetylcholine receptors in the nematode Caenorhabditis elegans. J. Mol. Biol. 258, 261-269.

Bennett, K.L., Bradshaw, J., Youngman, T., Rodgers, J., Greenfield, B., Aruffo, A., and Linsley, P.S. (1997). Deleted in colorectal carcinoma (DCC) binds heparin via its fifth fibronectin type III domain. J. Biol. Chem. 272, 26940-26946.

Blanchette, C.R., Perrat, P.N., Thackeray, A., and Bénard, C.Y. (2015). Glypican Is a Modulator of Netrin-Mediated Axon Guidance. PLoS Biol 13.

Blanchette, C.R., Thackeray, A., Perrat, P.N., Hekimi, S., and Bénard, C.Y. (2017). Functional Requirements for Heparan Sulfate Biosynthesis in Morphogenesis and Nervous System Development in C. elegans. PLoS Genet. 13, e1006525.

Bolte, S., and Cordelières, F.P. A guided tour into subcellular colocalization analysis in light microscopy. Journal of Microscopy 224, 213-232.

Brusés, J.L., Chauvet, N., and Rutishauser, U. (2001). Membrane lipid rafts are necessary for the maintenance of the (alpha)7 nicotinic acetylcholine receptor in somatic spines of ciliary neurons. $\mathrm{J}$. Neurosci. 21, 504-512.

Bülow, H.E., Berry, K.L., Topper, L.H., Peles, E., and Hobert, O. (2002). Heparan sulfate proteoglycan-dependent induction of axon branching and axon misrouting by the Kallmann syndrome gene kal-1. Proc. Natl. Acad. Sci. U.S.A. 99, 6346-6351.

Burden, S.J., Huijbers, M.G., and Remedio, L. (2018). Fundamental Molecules and Mechanisms for Forming and Maintaining Neuromuscular Synapses. Int J Mol Sci 19. 
Bürli, T., Baer, K., Ewers, H., Sidler, C., Fuhrer, C., and Fritschy, J.-M. (2010). Single particle tracking of alpha7 nicotinic $A C h R$ in hippocampal neurons reveals regulated confinement at glutamatergic and GABAergic perisynaptic sites. PLoS ONE 5, e11507.

Charpantier, E., Wiesner, A., Huh, K.-H., Ogier, R., Hoda, J.-C., Allaman, G., Raggenbass, M., Feuerbach, D., Bertrand, D., and Fuhrer, C. (2005). a7 Neuronal Nicotinic Acetylcholine Receptors Are Negatively Regulated by Tyrosine Phosphorylation and Src-Family Kinases. J Neurosci 25, 98369849.

Choi, S., Lee, E., Kwon, S., Park, H., Yi, J.Y., Kim, S., Han, I.-O., Yun, Y., and Oh, E.-S. (2005). Transmembrane Domain-induced Oligomerization Is Crucial for the Functions of Syndecan-2 and Syndecan-4. J. Biol. Chem. 280, 42573-42579.

Condomitti, G., and de Wit, J. (2018). Heparan Sulfate Proteoglycans as Emerging Players in Synaptic Specificity. Front Mol Neurosci 11, 14.

Condomitti, G., Wierda, K.D., Schroeder, A., Rubio, S.E., Vennekens, K.M., Orlandi, C., Martemyanov, K.A., Gounko, N.V., Savas, J.N., and de Wit, J. (2018). An input-specific orphan receptor GPR158HSPG interaction organizes hippocampal mossy fiber-CA3 synapses. Neuron 100, 201-215.e9.

Conroy, W.G., Liu, Z., Nai, Q., Coggan, J.S., and Berg, D.K. (2003). PDZ-Containing Proteins Provide a Functional Postsynaptic Scaffold for Nicotinic Receptors in Neurons. Neuron 38, 759-771.

Corti, F., Wang, Y., Rhodes, J.M., Atri, D., Archer-Hartmann, S., Zhang, J., Zhuang, Z.W., Chen, D., Wang, T., Wang, Z., et al. (2019). N-terminal syndecan-2 domain selectively enhances 6-O heparan sulfate chains sulfation and promotes VEGFA165-dependent neovascularization. Nat Commun 10, 1562.

Dani, N., and Broadie, K. (2012). Glycosylated synaptomatrix regulation of trans-synaptic signaling. Dev Neurobiol 72, 2-21.

Dawe, G.B., Yu, H., Gu, S., Blackler, A.N., Matta, J.A., Siuda, E.R., Rex, E.B., and Bredt, D.S. (2019). $\alpha 7$ nicotinic acetylcholine receptor upregulation by anti-apoptotic $\mathrm{Bcl}-2$ proteins. Nature Communications 10, 2746.

Dineley, K.T., Pandya, A.A., and Yakel, J.L. (2015). Nicotinic ACh receptors as therapeutic targets in CNS disorders. Trends Pharmacol. Sci. 36, 96-108.

Dityatev, A., and Rusakov, D.A. (2011). Molecular signals of plasticity at the tetrapartite synapse. Curr Opin Neurobiol 21, 353-359.

Dityatev, A., Frischknecht, R., and Seidenbecher, C.I. (2006). Extracellular matrix and synaptic functions. Results Probl Cell Differ 43, 69-97.

Dokshin, G.A., Ghanta, K.S., Piscopo, K.M., and Mello, C.C. (2018). Robust Genome Editing with Short Single-Stranded and Long, Partially Single-Stranded DNA Donors in Caenorhabditis elegans. Genetics 210, 781-787.

Dow, D.J., Huxley-Jones, J., Hall, J.M., Francks, C., Maycox, P.R., Kew, J.N.C., Gloger, I.S., Mehta, N.A.L., Kelly, F.M., Muglia, P., et al. (2011). ADAMTSL3 as a candidate gene for schizophrenia: Gene sequencing and ultra-high density association analysis by imputation. Schizophrenia Research 127 , 28-34.

Dunn, K.W., Kamocka, M.M., and McDonald, J.H. (2011). A practical guide to evaluating colocalization in biological microscopy. American Journal of Physiology - Cell Physiology 300, C723-C742.

Ethell, I.M., and Yamaguchi, Y. (1999). Cell Surface Heparan Sulfate Proteoglycan Syndecan-2 Induces the Maturation of Dendritic Spines in Rat Hippocampal Neurons. J Cell Biol 144, 575-586. 
Ethell, I.M., Hagihara, K., Miura, Y., Irie, F., and Yamaguchi, Y. (2000). Synbindin, a Novel Syndecan2-Binding Protein in Neuronal Dendritic Spines. J Cell Biol 151, 53-68.

Ethell, I.M., Irie, F., Kalo, M.S., Couchman, J.R., Pasquale, E.B., and Yamaguchi, Y. (2001). EphB/Syndecan-2 Signaling in Dendritic Spine Morphogenesis. Neuron 31, 1001-1013.

Fabian-Fine, R., Skehel, P., Errington, M.L., Davies, H.A., Sher, E., Stewart, M.G., and Fine, A. (2001). Ultrastructural distribution of the alpha7 nicotinic acetylcholine receptor subunit in rat hippocampus. J. Neurosci. 21, 7993-8003.

Farhy-Tselnicker, I., van Casteren, A.C.M., Lee, A., Chang, V.T., Aricescu, A.R., and Allen, N.J. (2017). Astrocyte-Secreted Glypican 4 Regulates Release of Neuronal Pentraxin 1 from Axons to Induce Functional Synapse Formation. Neuron 96, 428-445.e13.

Farías, G.G., Vallés, A.S., Colombres, M., Godoy, J.A., Toledo, E.M., Lukas, R.J., Barrantes, F.J., and Inestrosa, N.C. (2007). Wnt-7a induces presynaptic colocalization of alpha 7-nicotinic acetylcholine receptors and adenomatous polyposis coli in hippocampal neurons. J. Neurosci. 27, 5313-5325.

Ferrer-Ferrer, M., and Dityatev, A. (2018). Shaping Synapses by the Neural Extracellular Matrix. Front Neuroanat 12.

Finci, L.I., Krüger, N., Sun, X., Zhang, J., Chegkazi, M., Wu, Y., Schenk, G., Mertens, H.D.T., Svergun, D.I., Zhang, Y., et al. (2014). The crystal structure of netrin-1 in complex with DCC reveals the bifunctionality of netrin-1 as a guidance cue. Neuron $83,839-849$.

Francis, M.M., Evans, S.P., Jensen, M., Madsen, D.M., Mancuso, J., Norman, K.R., and Maricq, A.V. (2005). The Ror Receptor Tyrosine Kinase CAM-1 Is Required for ACR-16-Mediated Synaptic Transmission at the C. elegans Neuromuscular Junction. Neuron 46, 581-594.

Gally, C., Eimer, S., Richmond, J.E., and Bessereau, J.-L. (2004). A transmembrane protein required for acetylcholine receptor clustering in Caenorhabditis elegans. Nature 431, 578-582.

Gendrel, M., Rapti, G., Richmond, J.E., and Bessereau, J.-L. (2009). A secreted complement-controlrelated protein ensures acetylcholine receptor clustering. Nature 461, 992-996.

Gondelaud, F., and Ricard-Blum, S. (2019). Structures and interactions of syndecans. FEBS J. 286, 2994-3007.

Granés, F., Berndt, C., Roy, C., Mangeat, P., Reina, M., and Vilaró, S. (2003). Identification of a novel Ezrin-binding site in syndecan-2 cytoplasmic domain. FEBS Letters 547, 212-216.

Gray, R., Rajan, A.S., Radcliffe, K.A., Yakehiro, M., and Dani, J.A. (1996). Hippocampal synaptic transmission enhanced by low concentrations of nicotine. Nature $383,713-716$.

Halassa, M.M., Fellin, T., and Haydon, P.G. (2007). The tripartite synapse: roles for gliotransmission in health and disease. Trends in Molecular Medicine 13, 54-63.

Hancock, M.L., Canetta, S.E., Role, L.W., and Talmage, D.A. (2008). Presynaptic Type III Neuregulin1-ErbB signaling targets a7 nicotinic acetylcholine receptors to axons. J Cell Biol 181, 511521.

Heikkinen, A., Pihlajaniemi, T., Faissner, A., and Yuzaki, M. (2014). Neural ECM and synaptogenesis. Prog. Brain Res. 214, 29-51.

Hienola, A., Tumova, S., Kulesskiy, E., and Rauvala, H. (2006). N-syndecan deficiency impairs neural migration in brain. J Cell Biol 174, 569-580.

Horowitz, A., and Simons, M. (1998). Phosphorylation of the cytoplasmic tail of syndecan-4 regulates activation of protein kinase Calpha. J. Biol. Chem. 273, 25548-25551. 
Hsueh, Y.-P., and Sheng, M. (1999). Regulated Expression and Subcellular Localization of Syndecan Heparan Sulfate Proteoglycans and the Syndecan-Binding Protein CASK/LIN-2 during Rat Brain Development. J. Neurosci. 19, 7415-7425.

Hsueh, Y.-P., Yang, F.-C., Kharazia, V., Naisbitt, S., Cohen, A.R., Weinberg, R.J., and Sheng, M. (1998). Direct Interaction of CASK/LIN-2 and Syndecan Heparan Sulfate Proteoglycan and Their Overlapping Distribution in Neuronal Synapses. J Cell Biol 142, 139-151.

Hu, H.-T., Umemori, H., and Hsueh, Y.-P. (2016). Postsynaptic SDC2 induces transsynaptic signaling via FGF22 for bidirectional synaptic formation. Sci Rep 6.

Jang, B., Jung, H., Hong, H., and Oh, E.-S. (2018). Syndecan transmembrane domain modulates intracellular signaling by regulating the oligomeric status of the cytoplasmic domain. Cell. Signal. 52, $121-126$.

Jensen, M., Hoerndli, F.J., Brockie, P.J., Wang, R., Johnson, E., Maxfield, D., Francis, M.M., Madsen, D.M., and Maricq, A.V. (2012). Wnt signaling regulates acetylcholine receptor translocation and synaptic plasticity in the adult nervous system. Cell 149, 173-187.

Johnson, K.G., Tenney, A.P., Ghose, A., Duckworth, A.M., Higashi, M.E., Parfitt, K., Marcu, O., Heslip, T.R., Marsh, J.L., Schwarz, T.L., et al. (2006). The HSPGs Syndecan and Dallylike Bind the Receptor Phosphatase LAR and Exert Distinct Effects on Synaptic Development. Neuron 49, 517-531.

Kaksonen, M., Pavlov, I., Võikar, V., Lauri, S.E., Hienola, A., Riekki, R., Lakso, M., Taira, T., and Rauvala, H. (2002). Syndecan-3-Deficient Mice Exhibit Enhanced LTP and Impaired HippocampusDependent Memory. Molecular and Cellular Neuroscience 21, 158-172.

Kawai, H., Zago, W., and Berg, D.K. (2002). Nicotinic a7 Receptor Clusters on Hippocampal GABAergic Neurons: Regulation by Synaptic Activity and Neurotrophins. J Neurosci 22, 7903-7912.

Kurshan, P.T., Phan, A.Q., Wang, G.J., Crane, M.M., Lu, H., and Shen, K. (2014). Regulation of Synaptic Extracellular Matrix Composition Is Critical for Proper Synapse Morphology. J Neurosci 34, 12678-12689.

Li, L., Xiong, W.-C., and Mei, L. (2018). Neuromuscular Junction Formation, Aging, and Disorders. Annu. Rev. Physiol. 80, 159-188.

Liewald, J.F., Brauner, M., Stephens, G.J., Bouhours, M., Schultheis, C., Zhen, M., and Gottschalk, A. (2008). Optogenetic analysis of synaptic function. Nat Methods 5, 895-902.

Lin, Y.-L., Lei, Y.-T., Hong, C.-J., and Hsueh, Y.-P. (2007). Syndecan-2 induces filopodia and dendritic spine formation via the neurofibromin-PKA-Ena/VASP pathway. J Cell Biol 177, 829-841.

Lindahl, U., and Li, J. (2009). Interactions between heparan sulfate and proteins-design and functional implications. Int Rev Cell Mol Biol 276, 105-159.

Liu, Y., Ford, B., Mann, M.A., and Fischbach, G.D. (2001). Neuregulins increase alpha7 nicotinic acetylcholine receptors and enhance excitatory synaptic transmission in GABAergic interneurons of the hippocampus. J. Neurosci. 21, 5660-5669.

Maro, G.S., Gao, S., Olechwier, A.M., Hung, W.L., Liu, M., Özkan, E., Zhen, M., and Shen, K. (2015). MADD-4/Punctin and Neurexin Organize C. elegans GABAergic Postsynapses through Neuroligin. Neuron 86, 1420-1432.

Matsumoto, Y., Irie, F., Inatani, M., Tessier-Lavigne, M., and Yamaguchi, Y. (2007). Netrin-1/DCC signaling in commissural axon guidance requires cell-autonomous expression of heparan sulfate. J. Neurosci. 27, 4342-4350. 
Minniti, A.N., Labarca, M., Hurtado, C., and Brandan, E. (2004). Caenorhabditis elegans syndecan (SDN-1) is required for normal egg laying and associates with the nervous system and the vulva. Journal of Cell Science 117, 5179-5190.

Murakami, M., Horowitz, A., Tang, S., Ware, J.A., and Simons, M. (2002). Protein Kinase C (PKC) $\delta$ Regulates PKCa Activity in a Syndecan-4-dependent Manner. J. Biol. Chem. 277, 20367-20371.

Nguyen, M.U., Kwong, J., Chang, J., Gillet, V.G., Lee, R.M., and Johnson, K.G. (2016). The Extracellular and Cytoplasmic Domains of Syndecan Cooperate Postsynaptically to Promote Synapse Growth at the Drosophila Neuromuscular Junction. PLOS ONE 11, e0151621.

Oh, E.-S., Couchman, J.R., and Woods, A. (1997). Serine Phosphorylation of Syndecan-2 Proteoglycan Cytoplasmic Domain. Archives of Biochemistry and Biophysics 344, 67-74.

Parker, M.J., Zhao, S., Bredt, D.S., Sanes, J.R., and Feng, G. (2004). PSD93 regulates synaptic stability at neuronal cholinergic synapses. J. Neurosci. 24, 378-388.

Pataki, C.A., Couchman, J.R., and Brábek, J. (2015). Wnt Signaling Cascades and the Roles of Syndecan Proteoglycans. J. Histochem. Cytochem. 63, 465-480.

Pinan-Lucarré, B., Tu, H., Pierron, M., Cruceyra, P.I., Zhan, H., Stigloher, C., Richmond, J.E., and Bessereau, J.-L. (2014). C. elegans Punctin specifies cholinergic versus GABAergic identity of postsynaptic domains. Nature 511, 466-470.

Rapti, G., Richmond, J., and Bessereau, J.-L. (2011). A single immunoglobulin-domain protein required for clustering acetylcholine receptors in C. elegans. EMBO J 30, 706-718.

Rhiner, C., Gysi, S., Fröhli, E., Hengartner, M.O., and Hajnal, A. (2005). Syndecan regulates cell migration and axon guidance in C. elegans. Development 132, 4621-4633.

Rodríguez-Manzaneque, J.C., Carpizo, D., Plaza-Calonge, M. del C., Torres-Collado, A.X., Thai, S.N.M., Simons, M., Horowitz, A., and Iruela-Arispe, M.L. (2009). Cleavage of syndecan-4 by ADAMTS1 provokes defects in adhesion. Int. J. Biochem. Cell Biol. 41, 800-810.

Saied-Santiago, K., and Bülow, H.E. (2018). Diverse Roles for Glycosaminoglycans in Neural Patterning. Dev Dyn 247, 54-74.

Sarrazin, S., Lamanna, W.C., and Esko, J.D. (2011). Heparan Sulfate Proteoglycans. Cold Spring Harb Perspect Biol 3.

Seetharaman, A., Selman, G., Puckrin, R., Barbier, L., Wong, E., D’Souza, S.A., and Roy, P.J. (2011). MADD-4 Is a Secreted Cue Required for Midline-Oriented Guidance in Caenorhabditis elegans.

Developmental Cell 21, 669-680.

Song, Y.S., and Kim, E. (2013). Presynaptic Proteoglycans: Sweet Organizers of Synapse Development. Neuron 79, 609-611.

Swenarchuk, L.E. (2019). Nerve, Muscle, and Synaptogenesis. Cells 8.

Tikiyani, V., Li, L., Sharma, P., Liu, H., Hu, Z., and Babu, K. (2018). Wnt Secretion Is Regulated by the Tetraspan Protein HIC-1 through Its Interaction with Neurabin/NAB-1. Cell Rep 25, 1856-1871.e6.

Tong, X.-J., Hu, Z., Liu, Y., Anderson, D., and Kaplan, J.M. (2015). A network of autism linked genes stabilizes two pools of synaptic GABAA receptors. ELife 4, e09648.

Touroutine, D., Fox, R.M., Stetina, S.E.V., Burdina, A., Miller, D.M., and Richmond, J.E. (2005). acr-16 Encodes an Essential Subunit of the Levamisole-resistant Nicotinic Receptor at the Caenorhabditis elegans Neuromuscular Junction. J. Biol. Chem. 280, 27013-27021. 
bioRxiv preprint doi: https://doi.org/10.1101/2020.11.25.395806; this version posted November 26, 2020. The copyright holder for this preprint (which was not certified by peer review) is the author/funder, who has granted bioRxiv a license to display the preprint in perpetuity. It is made available under aCC-BY-NC-ND 4.0 International license.

Tu, H., Pinan-Lucarré, B., Ji, T., Jospin, M., and Bessereau, J.-L. (2015). C. elegans Punctin Clusters GABAA Receptors via Neuroligin Binding and UNC-40/DCC Recruitment. Neuron 86, 1407-1419.

de Wit, J., O'Sullivan, M.L., Savas, J.N., Condomitti, G., Caccese, M.C., Vennekens, K.M., Yates, J.R., and Ghosh, A. (2013). Unbiased Discovery of Glypican as a Novel Receptor for LRRTM4 in Regulating Excitatory Synapse Development. Neuron 79, 696-711.

Wonnacott, S. (1997). Presynaptic nicotinic ACh receptors. Trends in Neurosciences 20, 92-98.

Xu, D., and Esko, J.D. (2014). Demystifying heparan sulfate-protein interactions. Annu. Rev. Biochem. 83, 129-157.

Xuan, Z., Manning, L., Nelson, J., Richmond, J.E., Colón-Ramos, D.A., Shen, K., and Kurshan, P.T. (2017). Clarinet (CLA-1), a novel active zone protein required for synaptic vesicle clustering and release. Elife 6.

Yuzaki, M. (2018). Two Classes of Secreted Synaptic Organizers in the Central Nervous System. Annu. Rev. Physiol. 80, 243-262.

Zarei, M.M., Radcliffe, K.A., Chen, D., Patrick, J.W., and Dani, J.A. (1999). Distributions of nicotinic acetylcholine receptor $\alpha 7$ and $\beta 2$ subunits on cultured hippocampal neurons. Neuroscience 88,755 764.

Zhang, L., Ward, J.D., Cheng, Z., and Dernburg, A.F. (2015). The auxin-inducible degradation (AID) system enables versatile conditional protein depletion in C. elegans. Development 142, 4374-4384.

Zhou, X., and Bessereau, J.-L. (2019). Molecular Architecture of Genetically-Tractable GABA Synapses in C. elegans. Front Mol Neurosci 12, 304.

Zhou, X., Gueydan, M., Jospin, M., Ji, T., Valfort, A., Pinan-Lucarré, B., and Bessereau, J.-L. (2020). The netrin receptor UNC-40/DCC assembles a postsynaptic scaffold and sets the synaptic content of GABAA receptors. Nat Commun 11, 2674.

Zhou, X., Zeng, J., Ouyang, C., Luo, Q., Yu, M., Yang, Z., Wang, H., Shen, K., and Shi, A. A novel bipartite UNC-101/AP-1 $\mu 1$ binding signal mediates KVS-4/Kv2.1 somatodendritic distribution in Caenorhabditis elegans. FEBS Letters 590, 76-92. 
Figure 1. SDN-1 is enriched at NMJs.

A

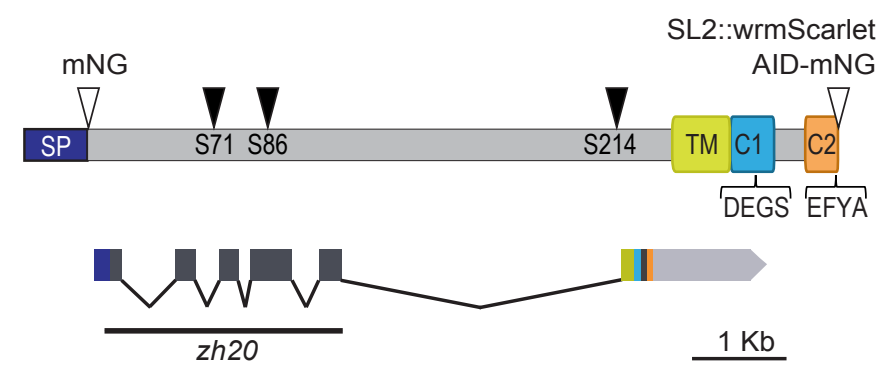

C
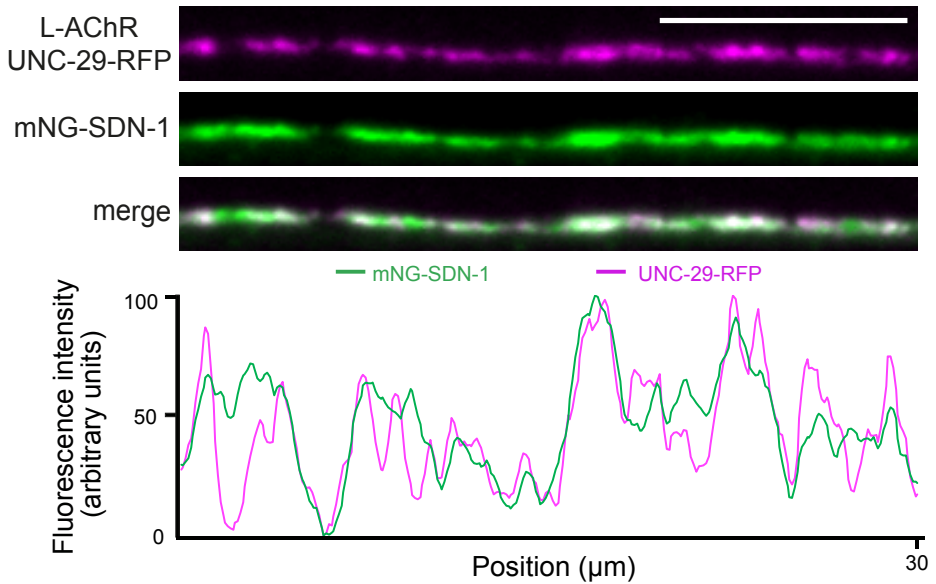

E
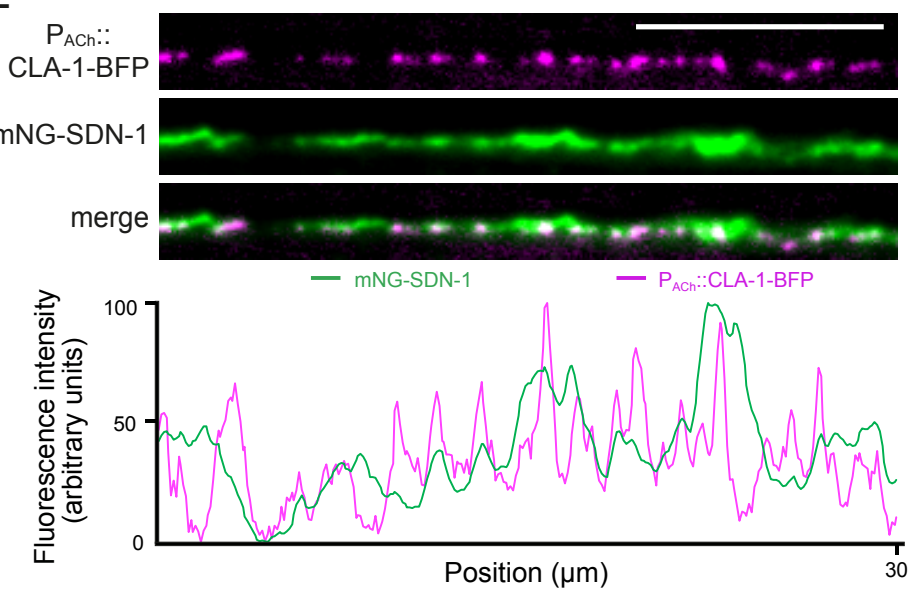

G

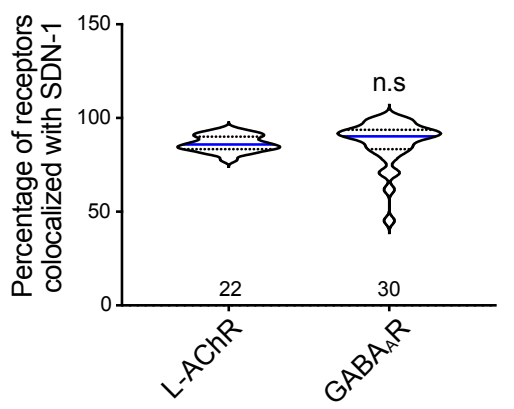

B

mNG-SDN-1

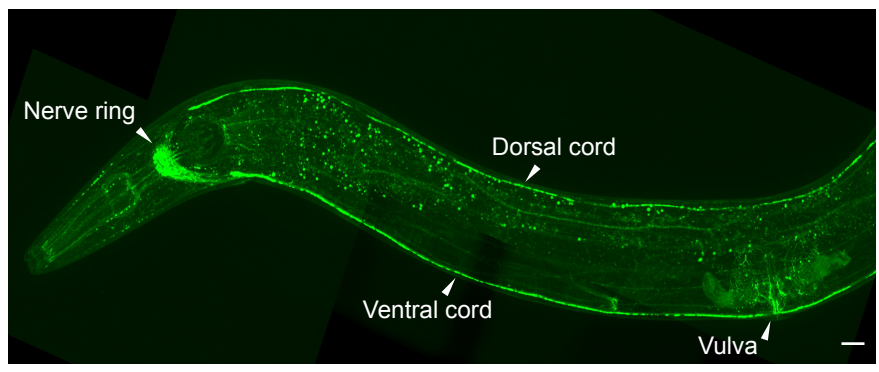

D
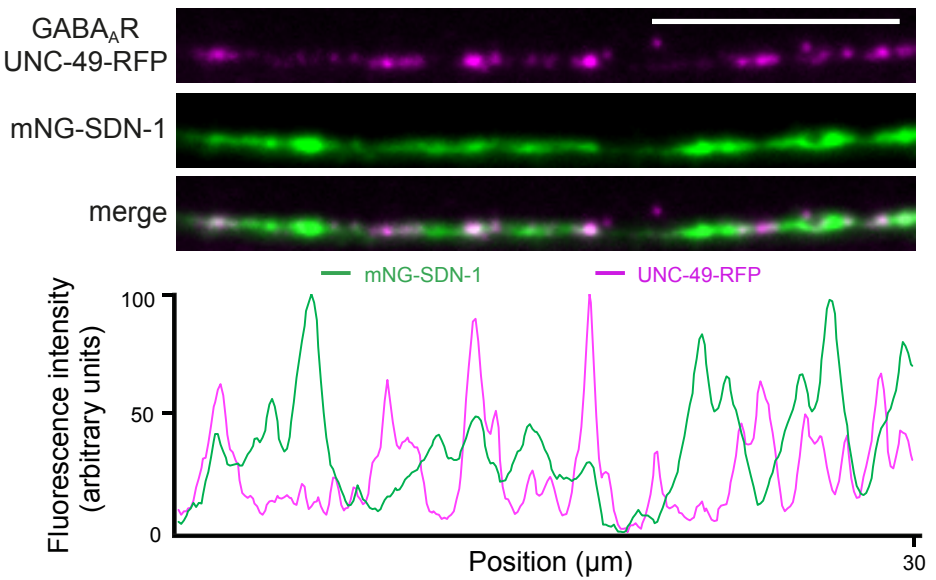

$F$ PGABA:: CLA-1-BFP mNG-SDN-1
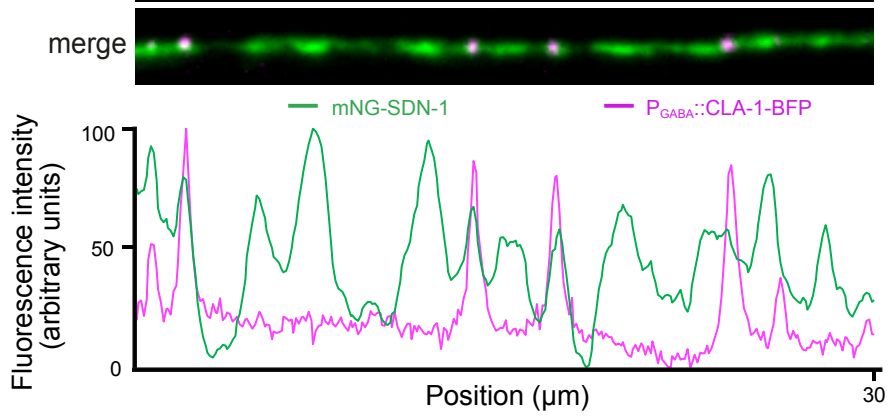

$\mathrm{H}$

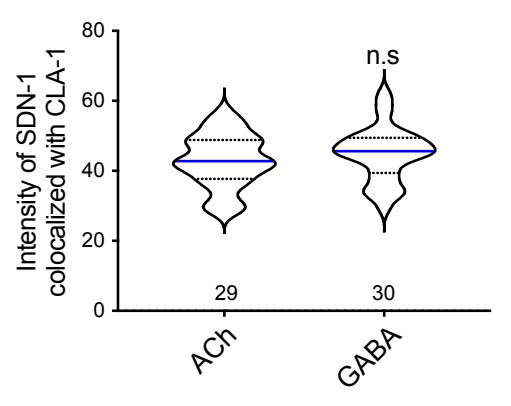


Figure 2. SDN-1 controls AChR synaptic content.

A

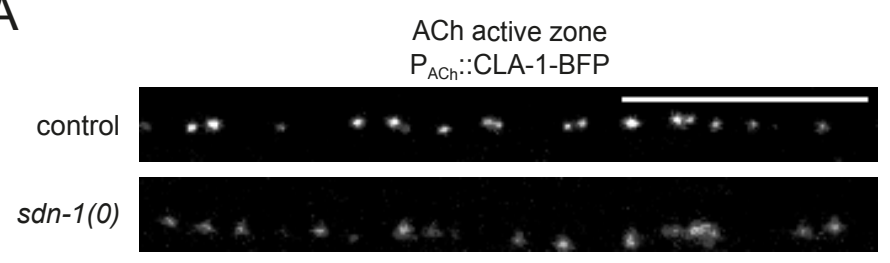

B

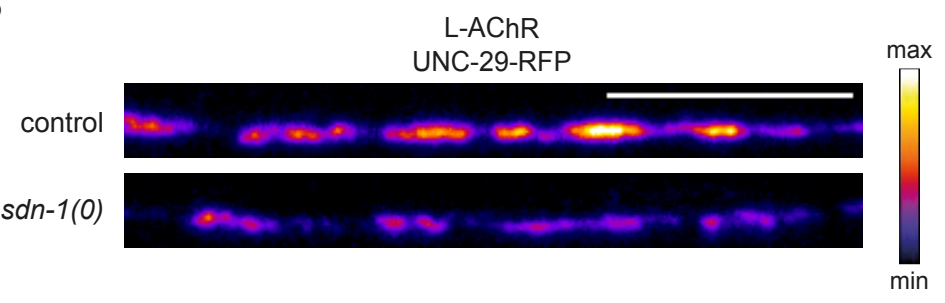

C

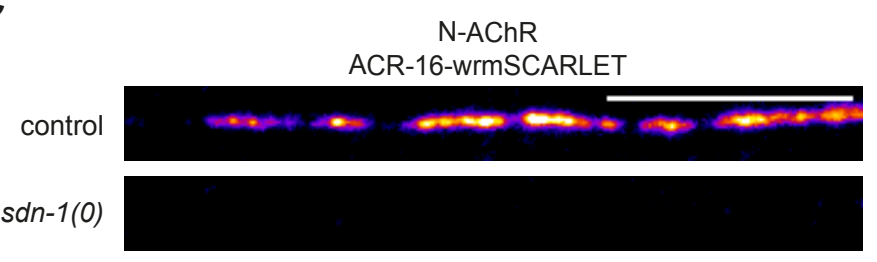

$\mathrm{D}$

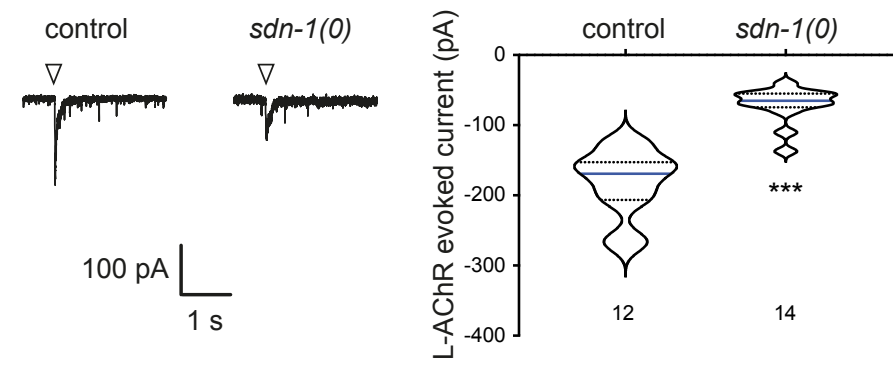

$\mathrm{F}$

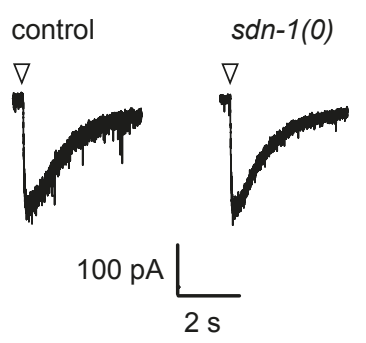

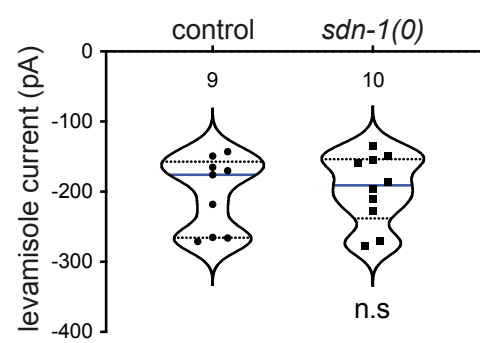
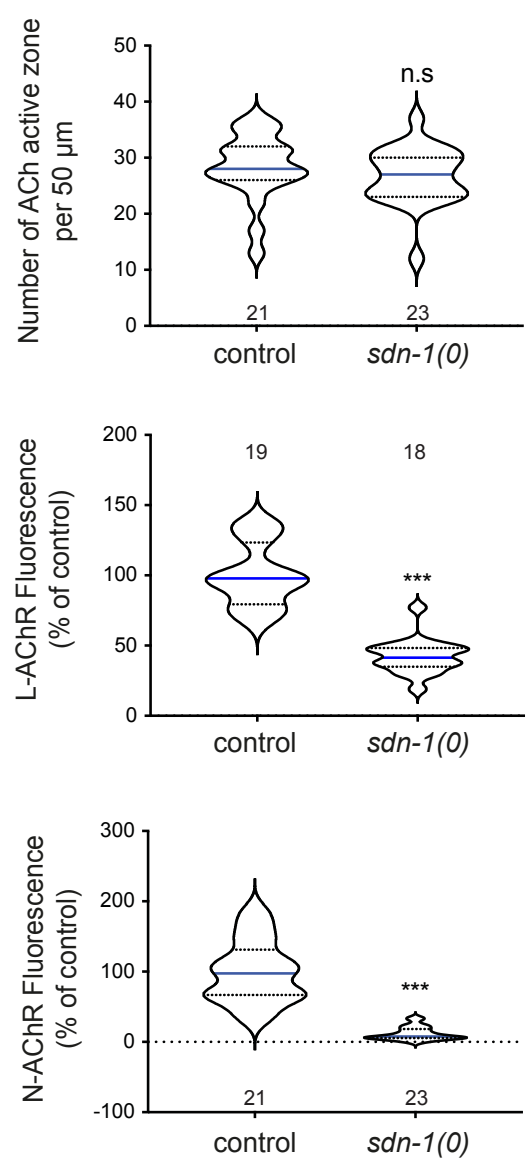

$\mathrm{E}$
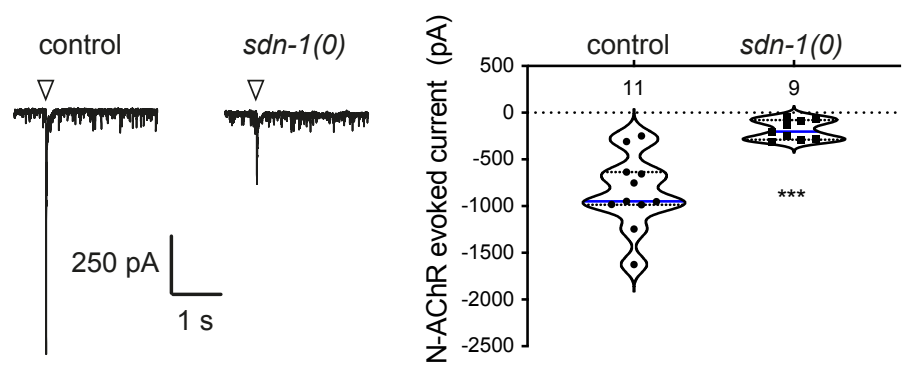

G

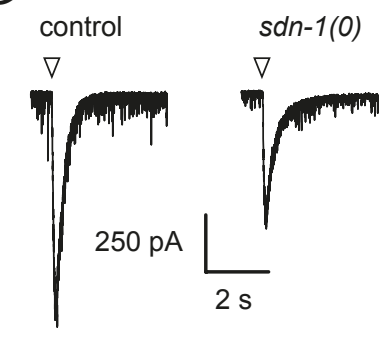




\section{Figure 3. Synaptic syndecan is mainly provided by postsynaptic muscle cells.}

A

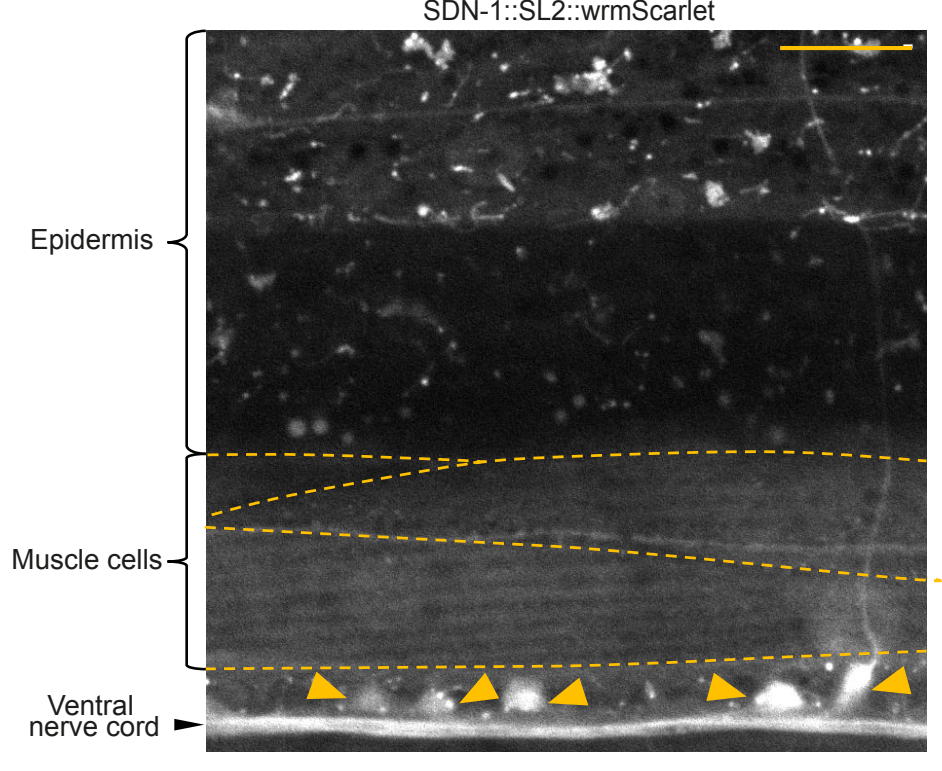

C

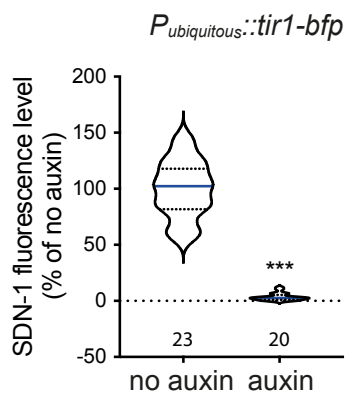

D

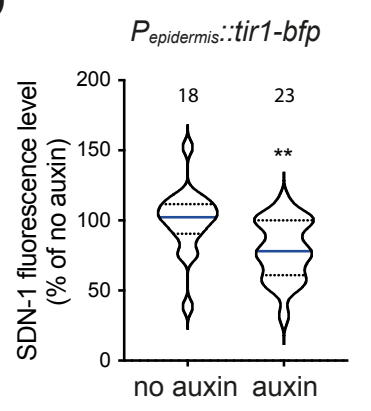

$\mathrm{B}$

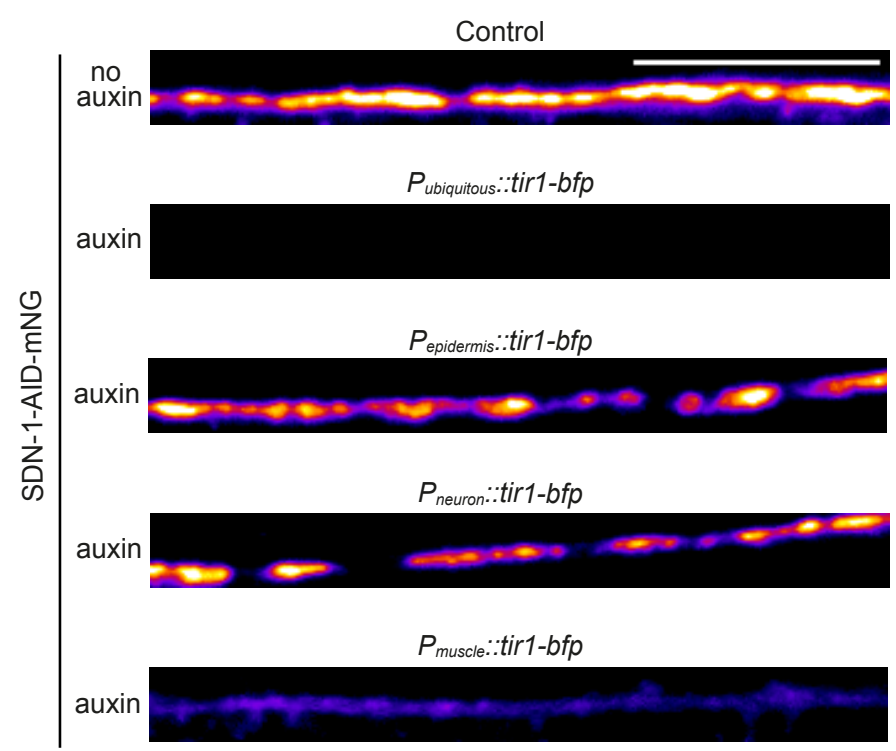

$E$

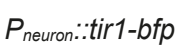

F

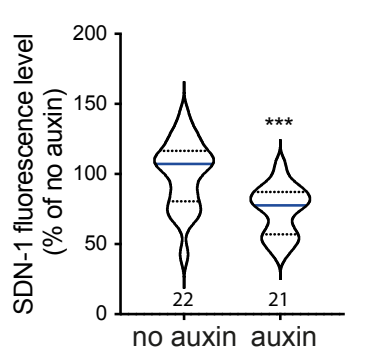

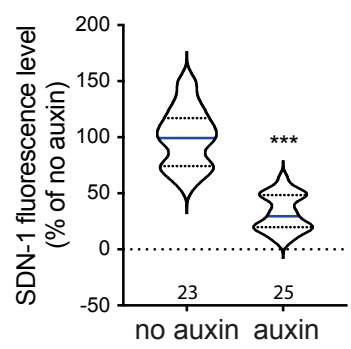

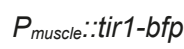


Figure 4. Ce-Punctin/MADD-4 localizes SDN-1 at synapses.

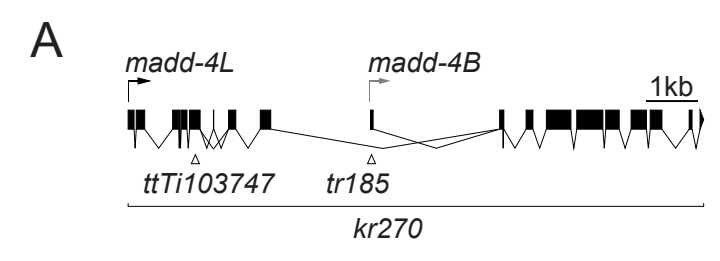

C
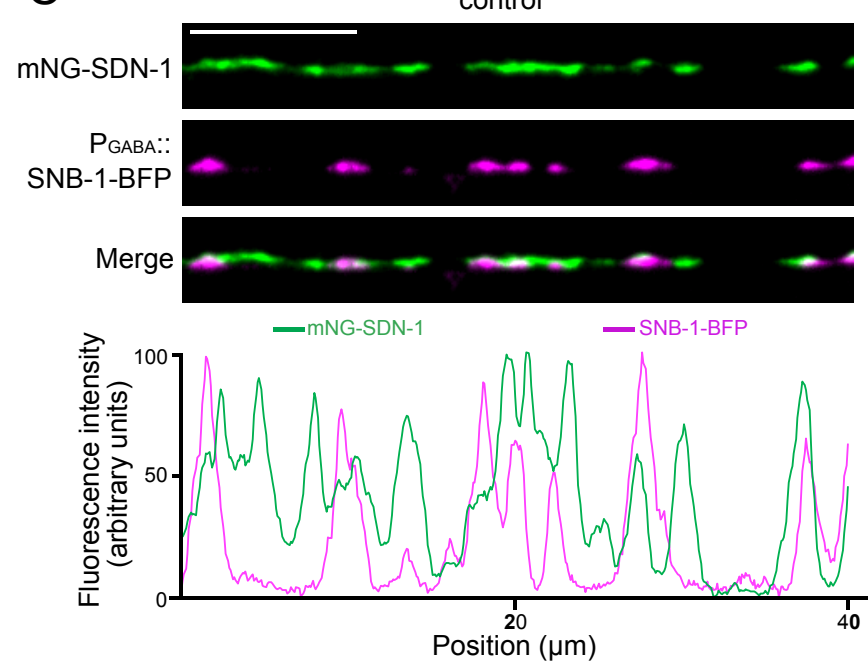

D

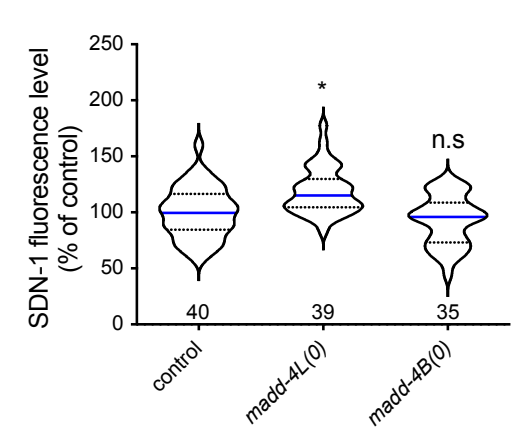

E

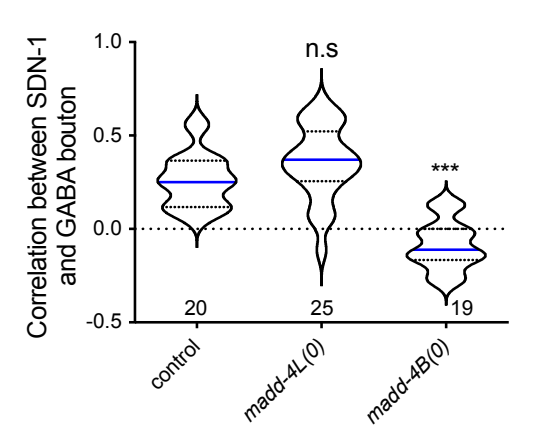

B
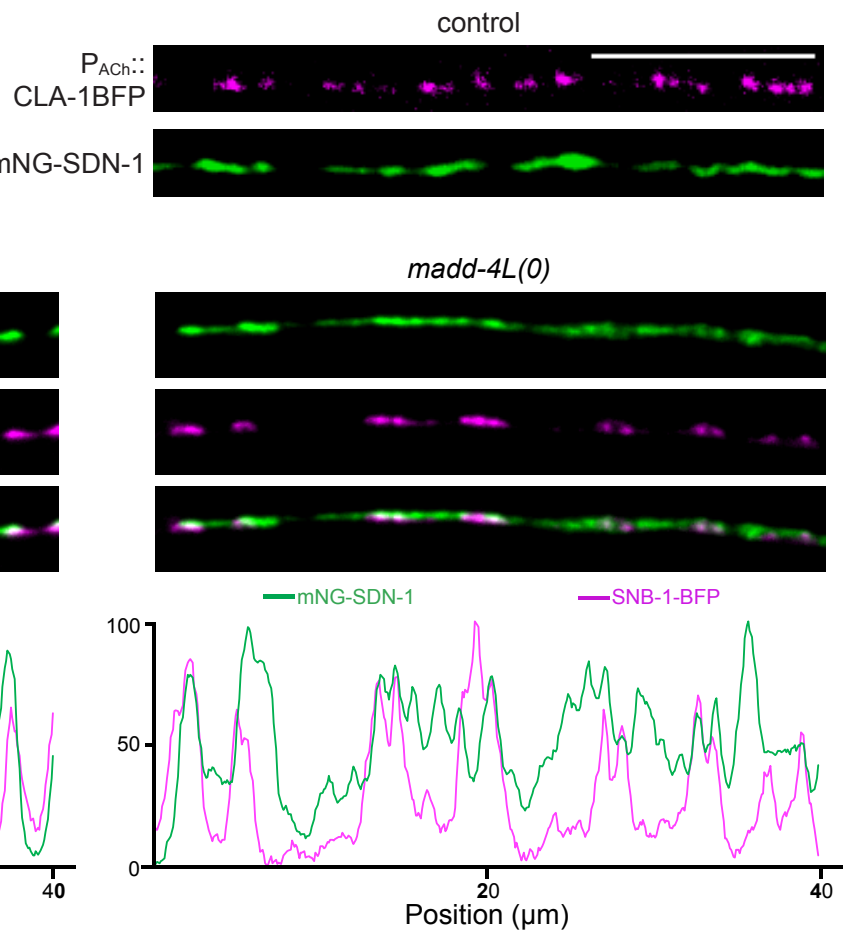

F

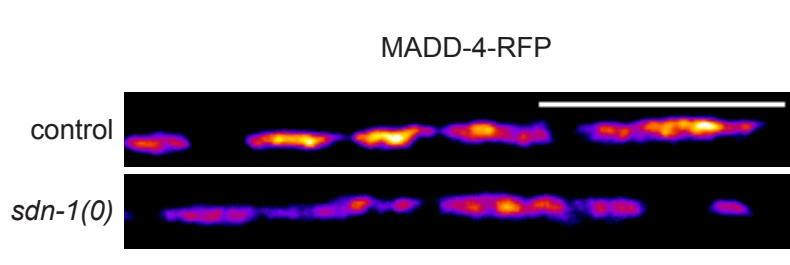

G

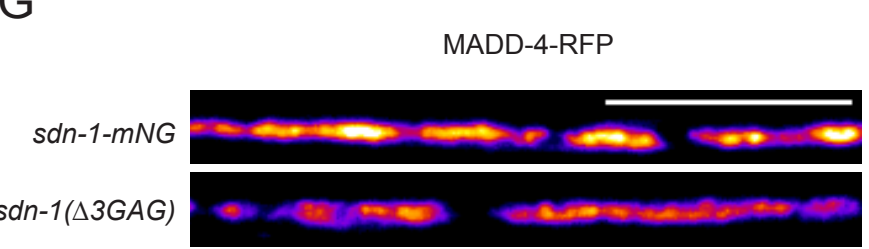

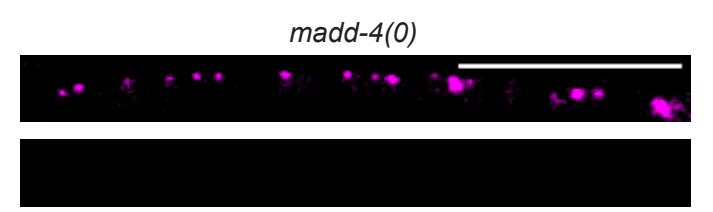
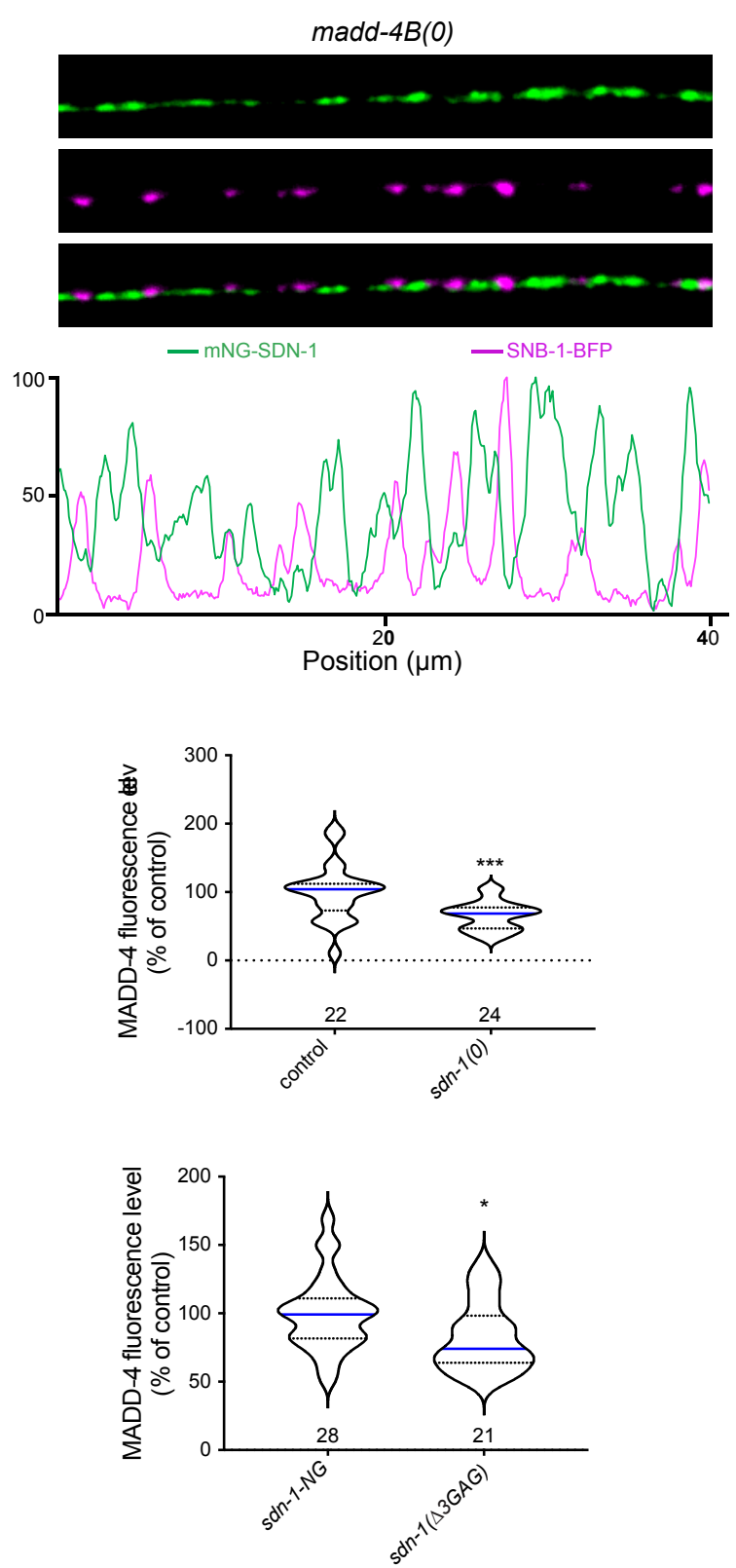
Figure 6. FRM-3/FARP bridges $\alpha 7$-like N-AChRs with LIN-2/CASK and SDN-1.

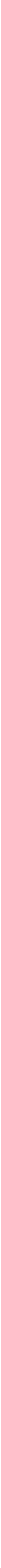


Figure 7. UNC-40 promotes the synaptic localization of ACR-16 and SDN-1.

A
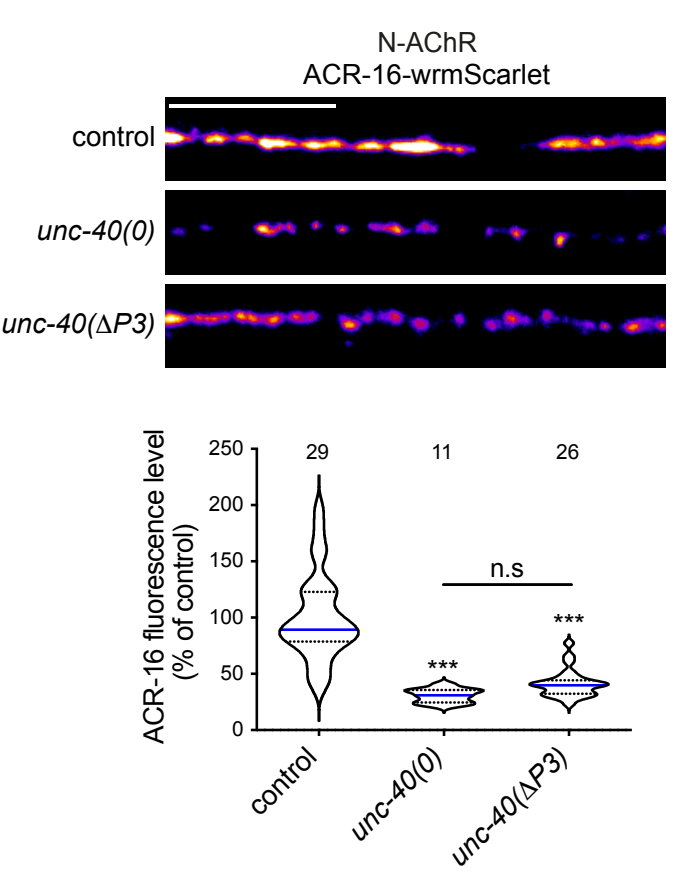

D
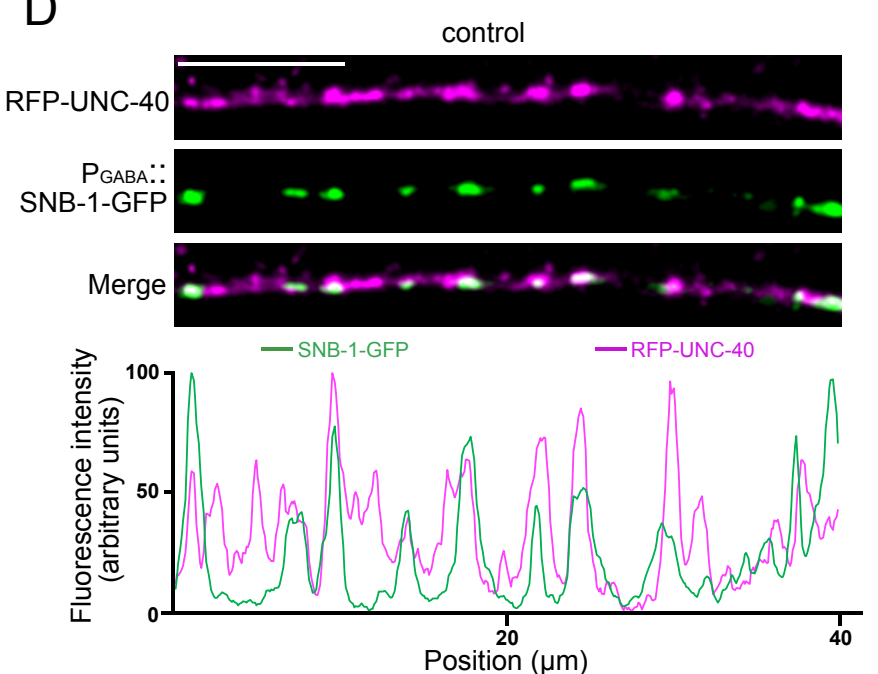

B
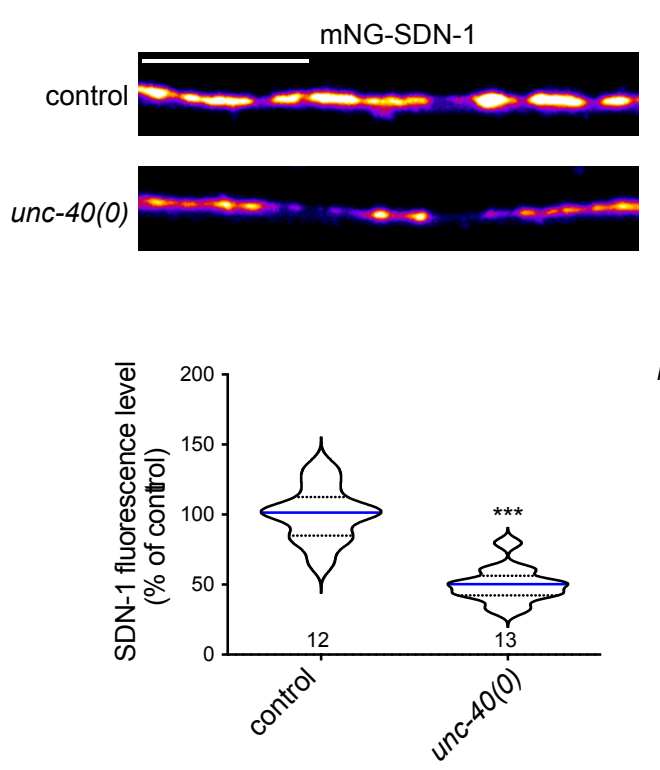

UNC-40 fluorescence level

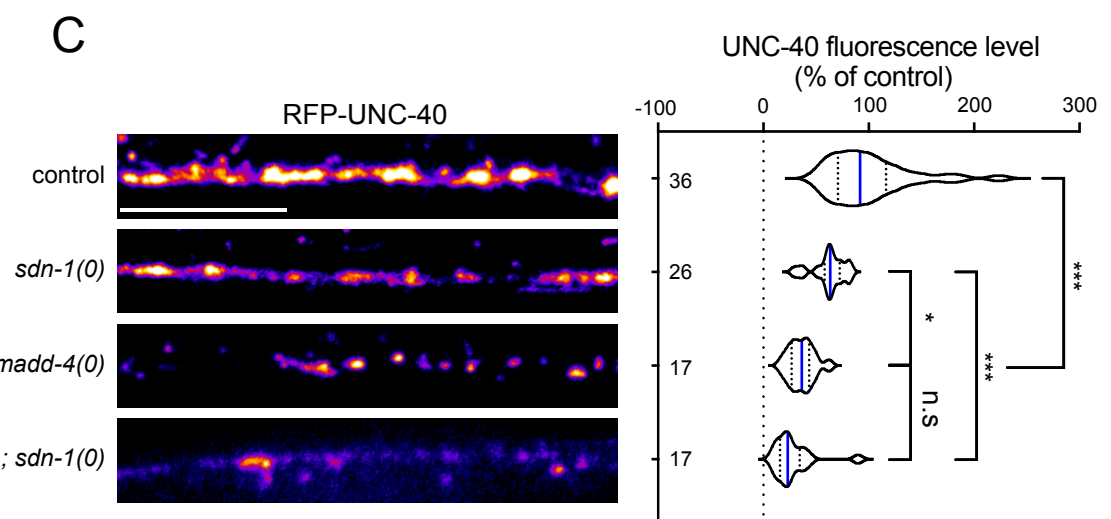

E
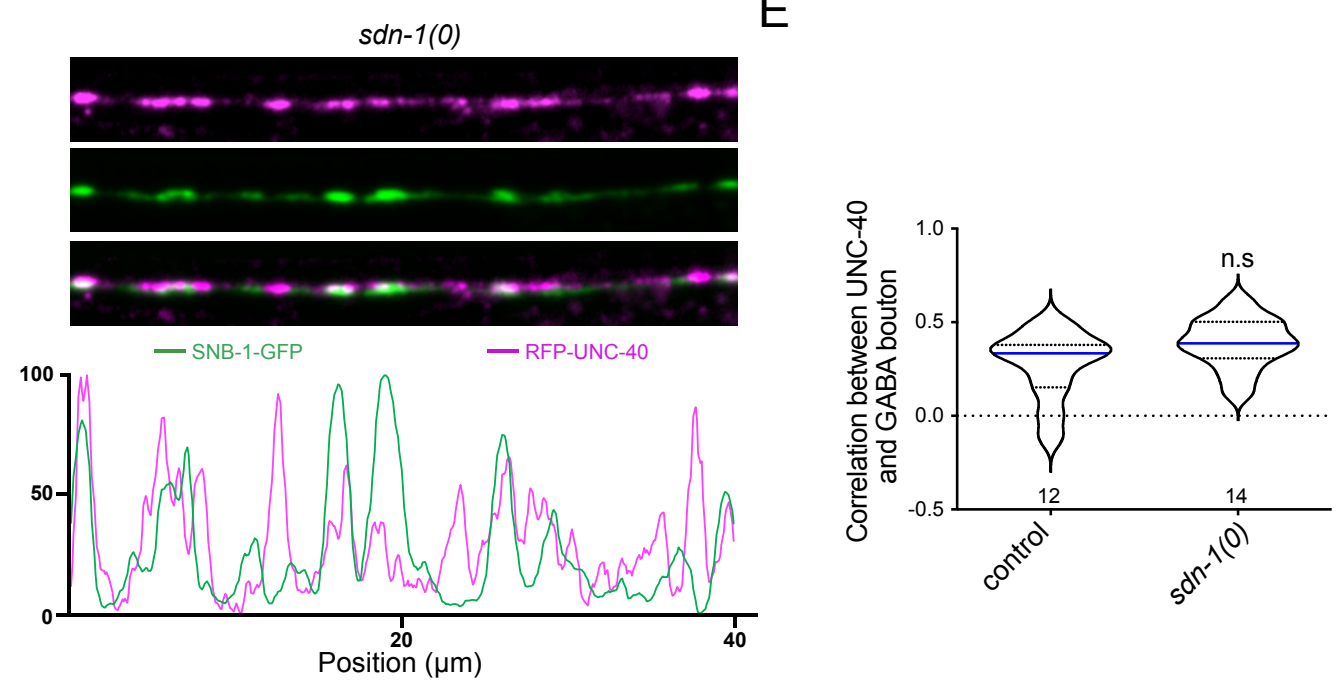
Figure 8. The intracellular domain of SDN-1 recruits N-AChR to GABA synapses.


F

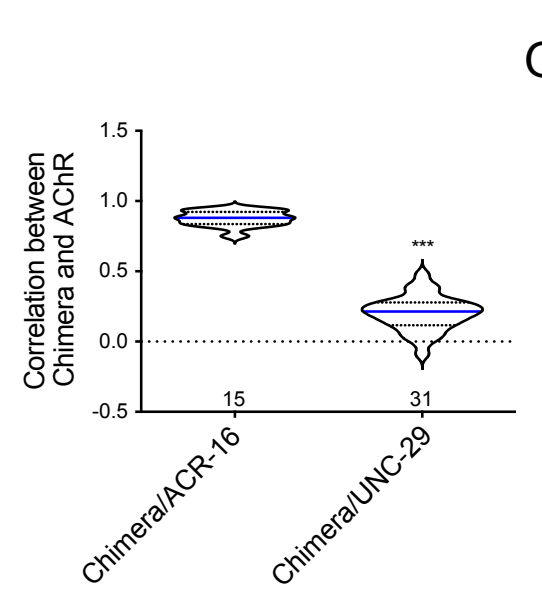

G
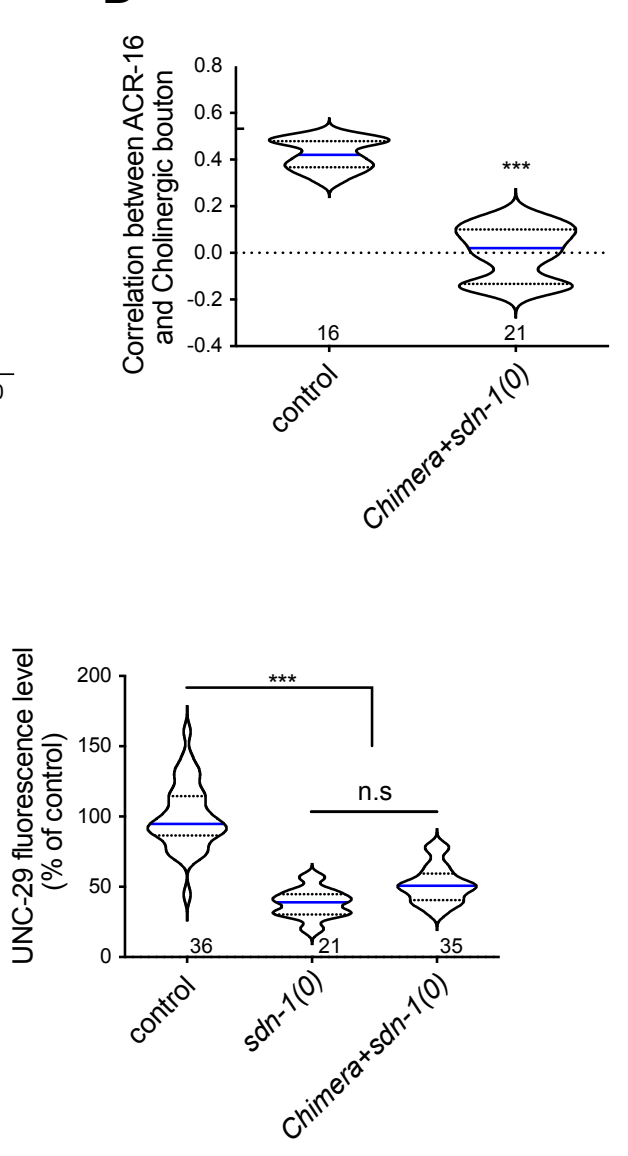

Pmyo-3::GFP-

PGaba::CLA-1-BFP

Merge

$P_{\text {myo-3::GFP- }}$

NLG-1ecd/SDN-1icd

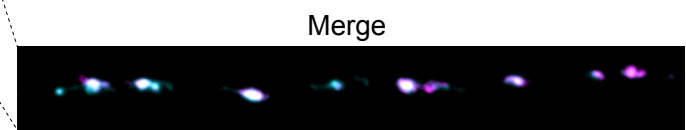

D

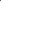


Figure 9. Working model for N-AChR clustering at NMJs.

Cholinergic presynaptic

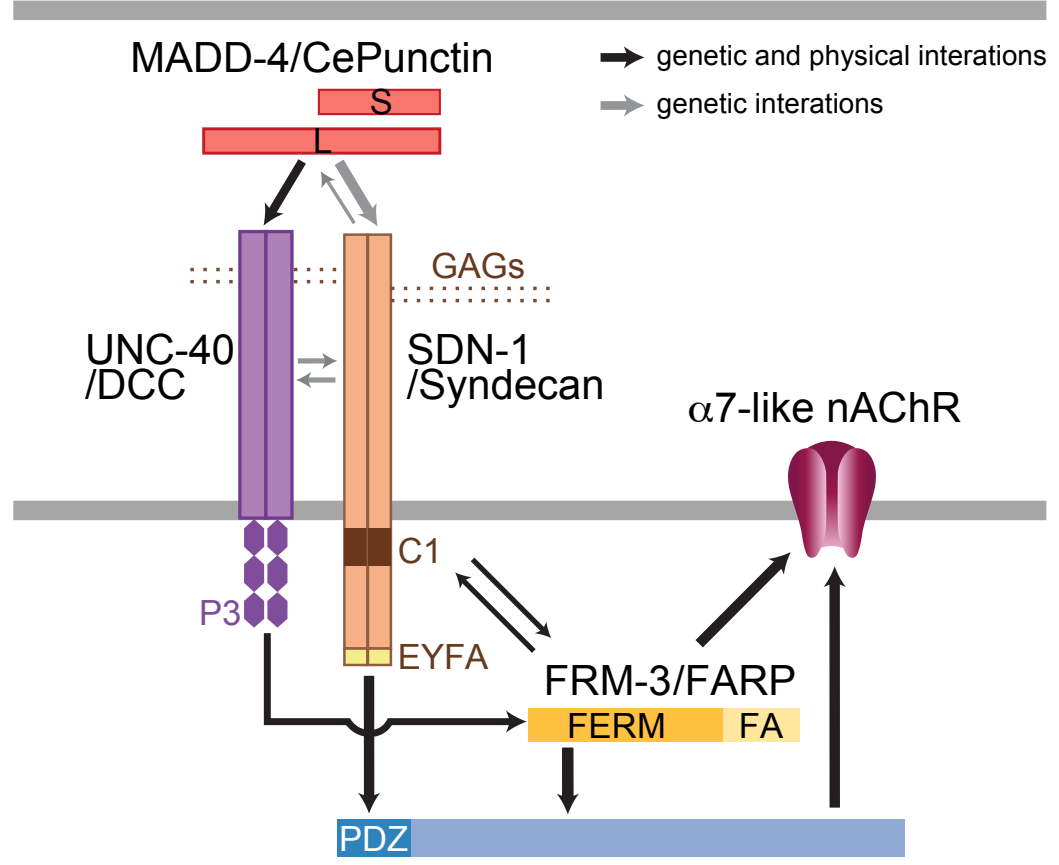

LIN-2/CASK

Muscle postsynaptic 
Figure S1. mNG insertion in the sdn-1 locus has no effect on NMJ organization.

A
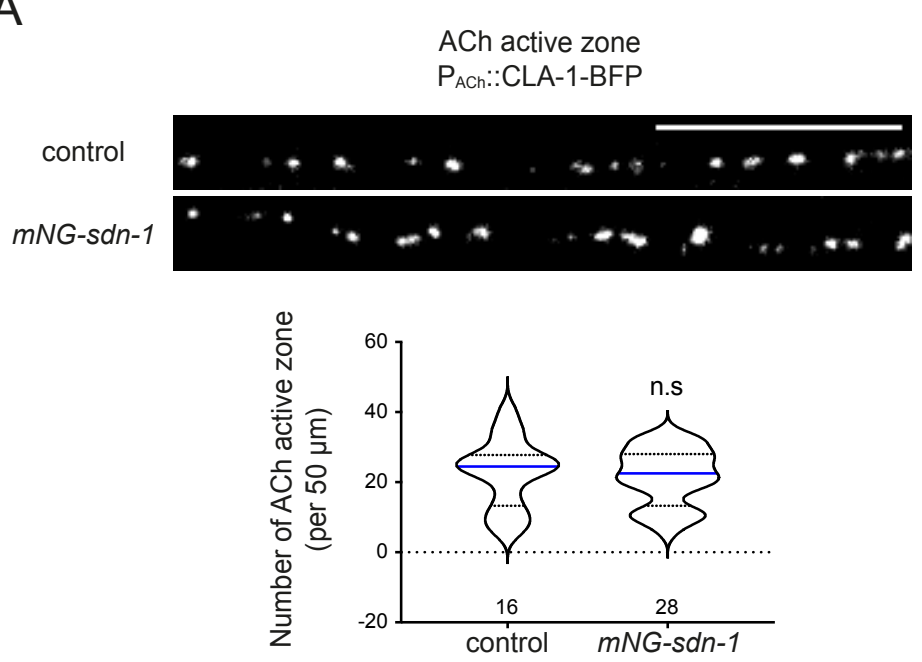

C
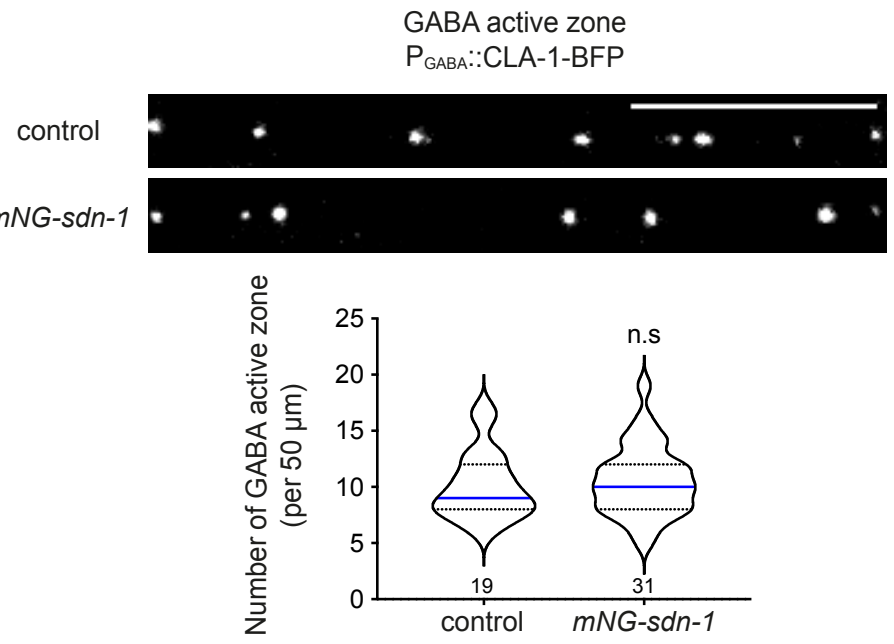

B

L-AChRs

UNC-29-RFP

control

$m N G-s d n-1$
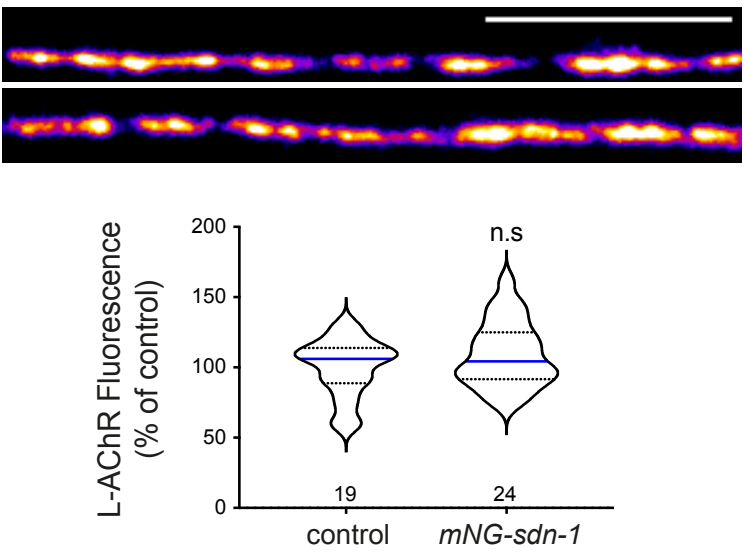

D

$\mathrm{GABA}_{\mathrm{A}} \mathrm{R}$

UNC-49-RFP

control

mNG-sdn-1
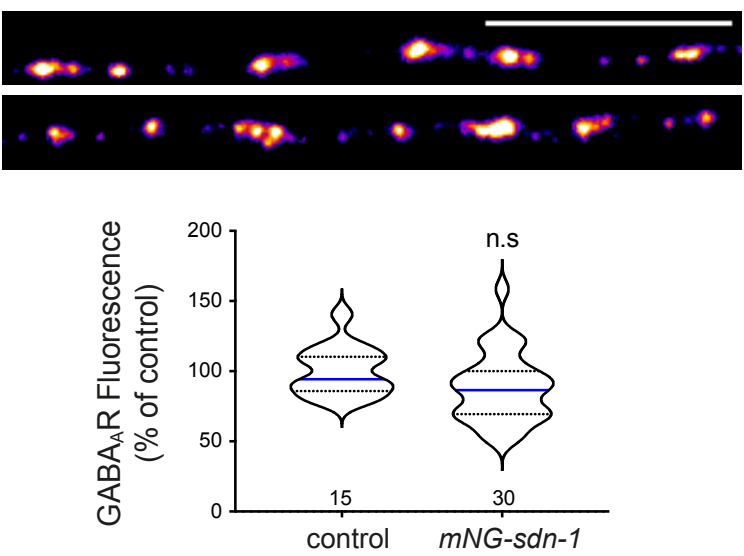
Figure S2. SDN-1 has a moderate effect on $\mathrm{GABA}_{\mathrm{A}} \mathrm{R}$ synaptic content.

A

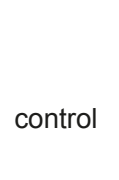

$s d n-1(0)$

$6,+\infty,+\infty, \quad+\infty$

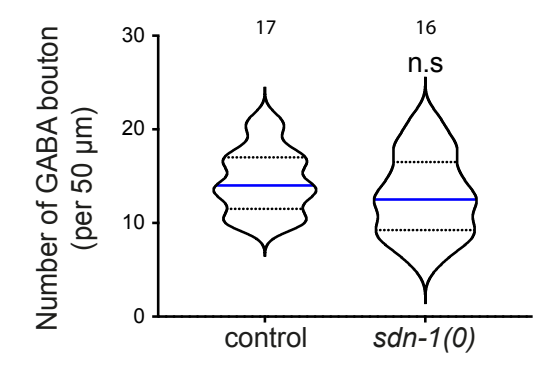
GABA boutons

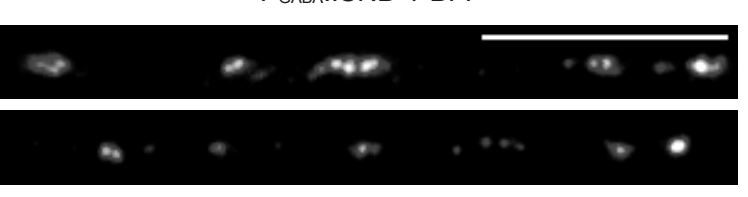

B

contro

$s d n-1(0)$

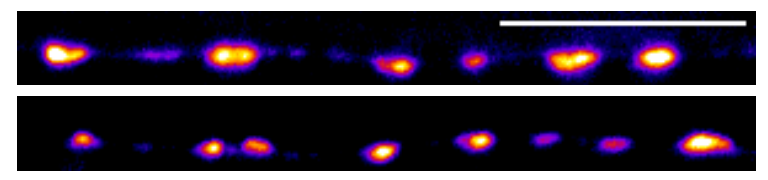

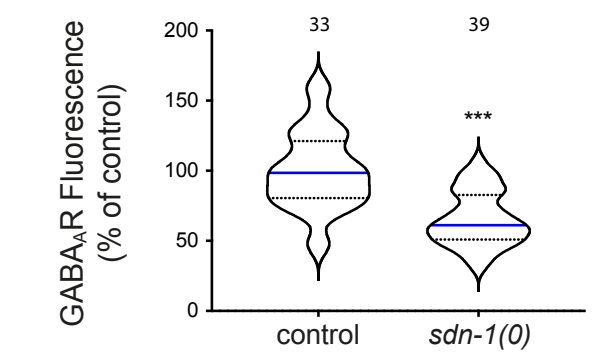

$\mathrm{GABA}_{A} R$

UNC-49-RFP 


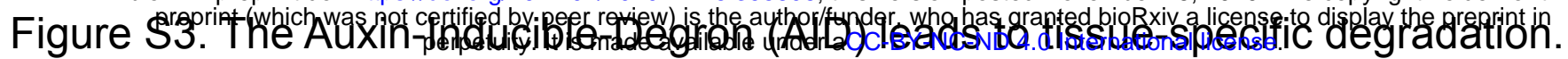

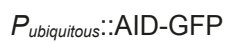

$P_{\text {ubiquitous::tir1-bfp }}$

no auxin

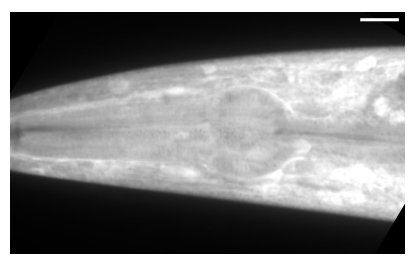

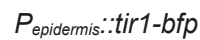

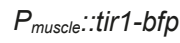

$P_{\text {neuron:: }: \text { ir1-bfp }}$
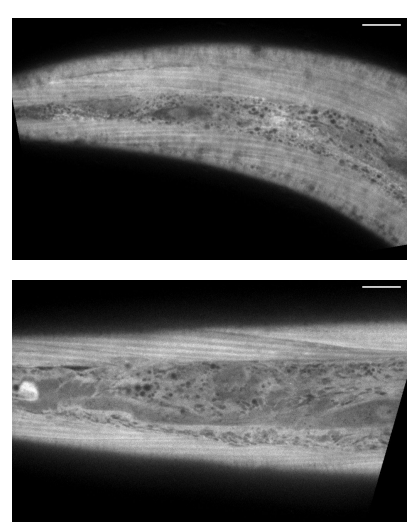
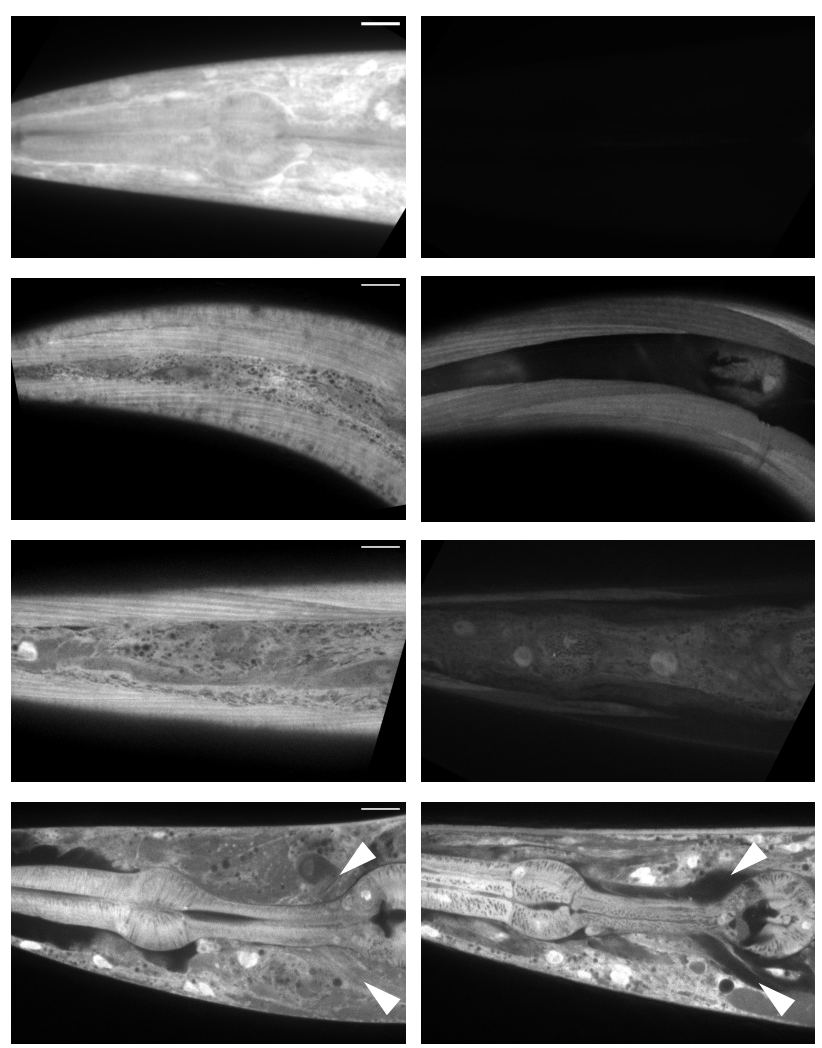

for its MADD-4-dependent synaptic localization.

A

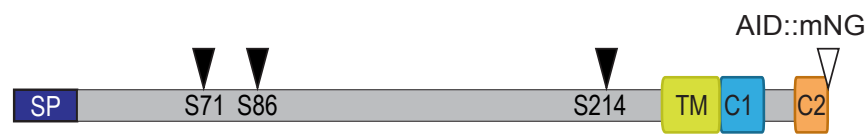

SDN-1( $\triangle 1 G A G):$ SDN-1(S71A)-mNG

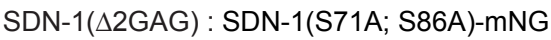

SDN-1( $\triangle 3 G A G)$ : SDN-1(S71A; S86A; S214A)-mNG

C

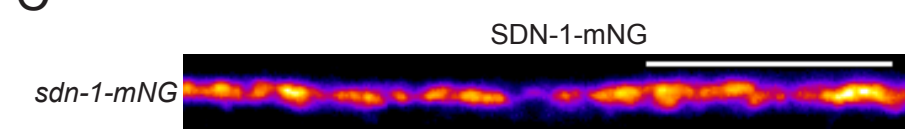

$\operatorname{sdn}-1(\triangle 1 G A G$

$s d n-1(\triangle 2 G A G$

$\operatorname{sdn}-1(\triangle 3 G A G$

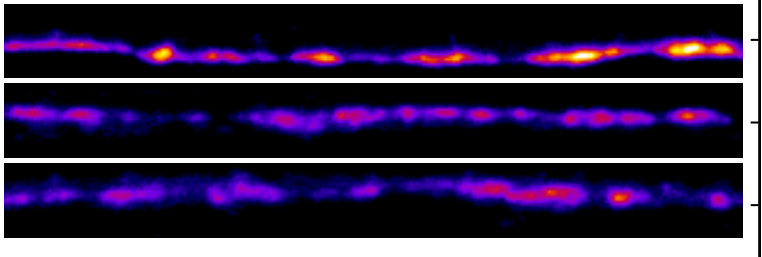

SDN-1 fluorescence level (\% of SDN-1-mNG)

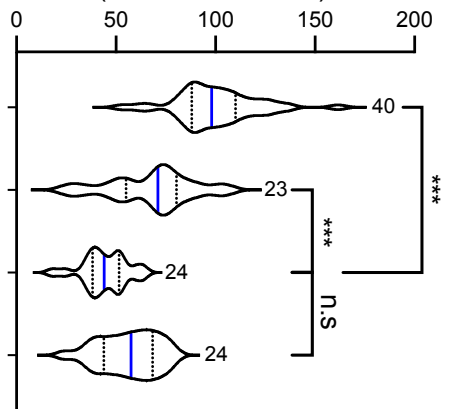

B
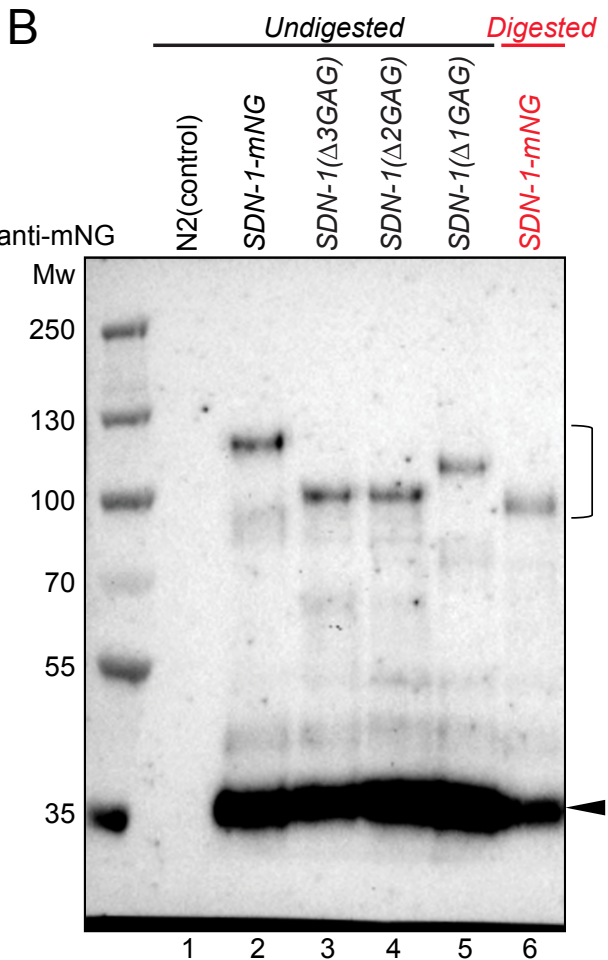
A
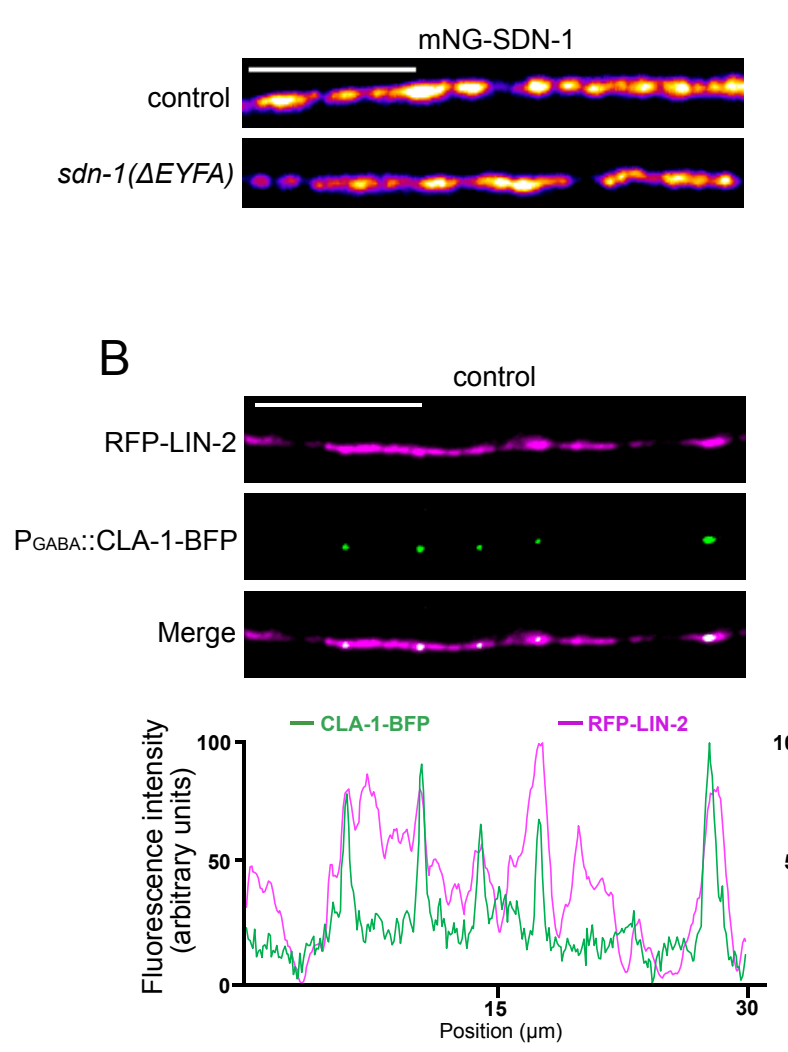

C

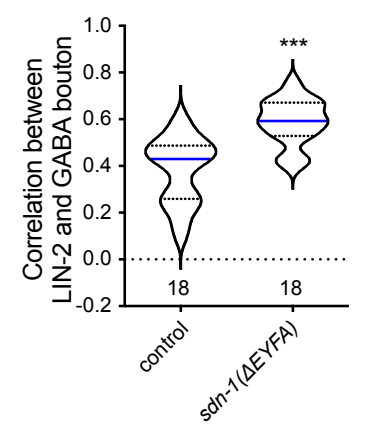

D
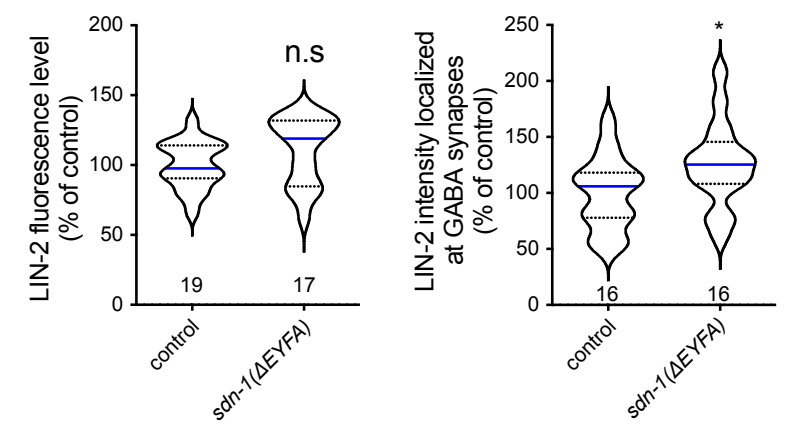